\title{
Numerical Methods for Solid Mechanics on Overlapping Grids: Linear Elasticity
}

\author{
Daniel Appelö ${ }^{1,1}$, Jeffrey W. Banks ${ }^{\mathrm{b}, 2}$, William D. Henshaw ${ }^{\mathrm{b}, 2, *}$, Donald W. Schwendeman ${ }^{\mathrm{c}, 3}$ \\ ${ }^{a}$ Department of Mathematics and Statistics, The University of New Mexico, 1 University of New Mexico, Albuquerque, NM \\ 87131, USA \\ ${ }^{b}$ Centre for Applied Scientific Computing, Lawrence Livermore National Laboratory, Livermore, CA 94551, USA \\ ${ }^{c}$ Department of Mathematical Sciences, Rensselaer Polytechnic Institute, Troy, NY 12180, USA
}

\begin{abstract}
This paper presents a new computational framework for the simulation of solid mechanics on general overlapping grids with adaptive mesh refinement (AMR). The approach, described here for time-dependent linear elasticity in two and three space dimensions, is motivated by considerations of accuracy, efficiency and flexibility. We consider two approaches for the numerical solution of the equations of linear elasticity on overlapping grids. In the first approach we solve the governing equations numerically as a second-order system (SOS) using a conservative finite-difference approximation. The second approach considers the equations written as a first-order system (FOS) and approximates them using a second-order characteristic-based (Godunov) finite-volume method. A principal aim of the paper is to present the first careful assessment of the accuracy and stability of these two representative schemes for the equations of linear elasticity on overlapping grids. This is done by first performing a stability analysis of analogous schemes for the first-order and second-order scalar wave equations on an overlapping grid. The analysis shows that non-dissipative approximations can have unstable modes with growth rates proportional to the inverse of the mesh spacing. This new result, which is relevant for the numerical solution of any type of wave propagation problem on overlapping grids, dictates the form of dissipation that is needed to stabilize the scheme. Numerical experiments show that the addition of the indicated form of dissipation and/or a separate filter step can be used to stabilize the SOS scheme. They also demonstrate that the upwinding inherent in the Godunov scheme, which provides dissipation of the appropriate form, stabilizes the FOS scheme. We then verify and compare the accuracy of the two schemes using the method of analytic solutions and using problems with known solutions. These latter problems provide useful benchmark solutions for time dependent elasticity. We also consider two problems in which exact solutions are not available, and use a posterior error estimates to assess the accuracy of the schemes. One of these two problems is additionally employed to demonstrate the use of dynamic AMR and its effectiveness for resolving elastic "shock" waves. Finally, results are presented that compare the computational performance of the two schemes. These demonstrate the speed and memory efficiency achieved by the use of structured overlapping grids and optimizations for Cartesian grids.
\end{abstract}

Keywords: Solid mechanics, linear elasticity, overlapping grids, numerical stability, finite difference, Godunov method

\section{Contents}

*Corresponding author. Mailing address: Centre for Applied Scientific Computing, L-422, Lawrence Livermore National Laboratory, Livermore, CA 94551, USA. Phone: 925-423-2697. Fax: 925-424-2477.

Email addresses: appelo@caltech.edu (Daniel Appelö), banks20@llnl.gov (Jeffrey W. Banks), henshaw1@llnl.gov

(William D. Henshaw), schwed@rpi.edu (Donald W. Schwendeman)

${ }^{1}$ This work supported by SKOJ Grant 64 18-21-12-5-19.

${ }^{2}$ This work was performed under the auspices of the U.S. Department of Energy (DOE) by Lawrence Livermore National Laboratory under Contract DE-AC52-07NA27344 and by DOE contracts from the ASCR Applied Math Program.

${ }^{3}$ This research was supported by Lawrence Livermore National Laboratory under subcontract B548468, and by the National Science Foundation under grants DMS-0532160 and DMS-0609874. 
3 Overlapping grid framework 6

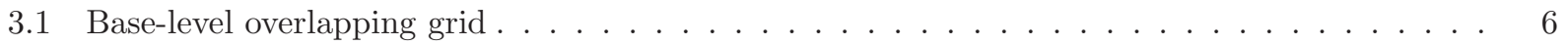

3.2 Adaptive mesh refinement . . . . . . . . . . . . . . . . . . . . . . . . 7

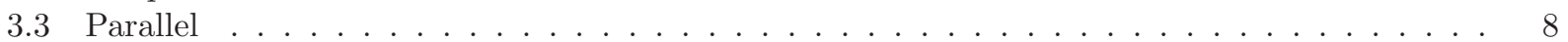

4 Discretization of the governing equations $\quad 8$

4.1 Second-order system $\ldots \ldots \ldots \ldots \ldots \ldots \ldots \ldots$

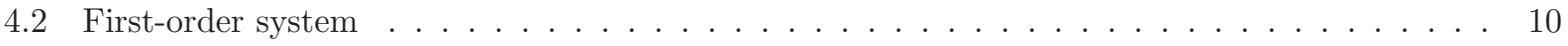

5 Stability at overlapping grid interfaces with nearby boundaries 12

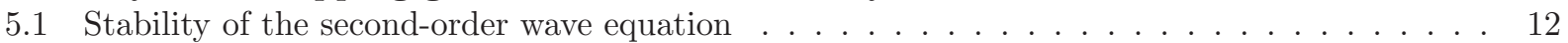

5.1 .1 An unstable solution for the second-order wave equation . . . . . . . . . . . . 15

5.1 .2 Artificial dissipation and high-order filtering . . . . . . . . . . . . . . . . . 16

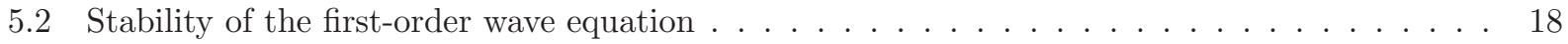

5.2 .1 Unstable solutions for the first-order wave equation . . . . . . . . . . . . . 19

5.2 .2 Dissipation in the Godunov method . . . . . . . . . . . . . . . . . 20

6 Numerical results $\quad 21$

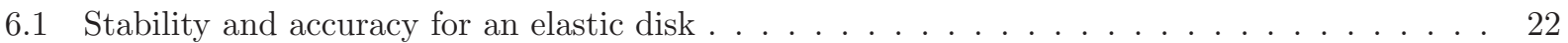

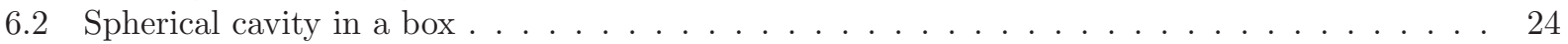

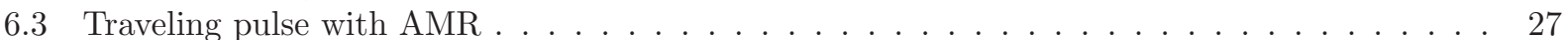

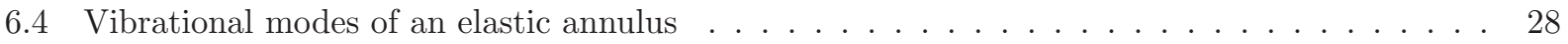

6.5 Vibrational modes of an elastic sphere . . . . . . . . . . . . . . . . . . . . 29

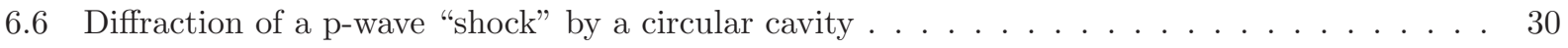

6.7 Three-dimensional circular plate with holes $\ldots \ldots \ldots \ldots \ldots$

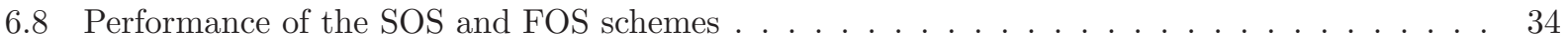

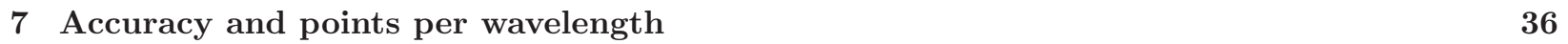

$\begin{array}{lll}8 & \text { Conclusions } & 37\end{array}$

$\begin{array}{lll}\text { Appendix A } & 38\end{array}$

Appendix A.1 Boundary conditions for the second-order system . . . . . . . . . . . 38

Appendix A.2 Boundary conditions for the first-order system $\ldots \ldots \ldots \ldots$

$\begin{array}{llll}\text { Appendix B } & \text { Artificial dissipation for the second-order system }\end{array}$

$\begin{array}{llll}\text { Appendix } & \mathrm{C} & \text { The method of analytic solutions } & 41\end{array}$

Appendix D Exact solutions to the elastic wave equation for an annulus and a sphere. 41

Appendix D.1 Vibrational modes of an elastic annulus - exact solutions . . . . . . . . . . . 42

Appendix D.2 Vibrational modes of an elastic sphere - exact solutions . . . . . . . . . 42

\section{Introduction}

The simulation of the deformation of solids is an important and well established field. A variety of numerical approaches have been used for such simulations, including finite element, finite difference, finite volume, spectral element and discontinuous Galerkin (DG) methods. For a review of finite difference, finite element and spectral element methods for wave equations, including the elastic wave equation, see [1]. Discontiuous Galerkin methods are newer and these have also been applied to the elastic wave equation, see for example $[2,3,4]$, and the references therein. Each approach has its strengths and weaknesses in terms of computational efficiency and accuracy, and the ability of the method to treat complex geometry, material interfaces, and use locally refined meshes. Finite difference methods on Cartesian grids, for example, are very efficient but may have difficulties in accurately treating complex geometry. Finite element, finite volume and DG methods based on unstructured grids can flexibly treat complex geometry ${ }^{4}$ but are more expensive

\footnotetext{
${ }^{4}$ The generation of unstructured hexahedral meshes for complex configurations, as required by some classes of schemes, can often be quite difficult.
} 
than approximations based on structured and Cartesian grids. The use of high-order accurate methods have great benefit for problems where waves propagate long distances. High-order accurate methods for general meshes may, however, be expensive, require significant storage and a reduced time step. In addition highorder methods generally work best when used on smooth high-quality meshes; but these may be difficult to generate for complex geometry.

In this work, we consider the deformation of solids using finite difference and finite volume methods on composite overlapping grids with adaptive mesh refinement (AMR). We have developed a new, and we believe unique, computational capability ${ }^{5}$ for problems of solid mechanics on general overlapping grids with AMR. Our approach, described here for linear elasticity, is motivated by considerations of accuracy, efficiency and flexibility. Overlapping grids can represent complex geometry using smooth boundary fitted structured grids. Smooth grids are especially important for obtaining accurate solutions to wave propagation problems. Our solution algorithms have the potential to be nearly as fast and memory efficient as schemes based on Cartesian grids and thus could have significant performance advantages over methods based on unstructured grids. For example, the cost per grid point in three dimensions of the Cartesian grid version of our scheme for the second-order system (described below) is approximately 10 times faster than the version for general curvilinear grids. This performance difference can be partially explained by comparing the cost to evaluate an approximation to a first or second derivative on a Cartesian grid, to that for a curvilinear grid. For a scheme with order of accuracy $p$, the finite difference stencil for a centered approximation is $p+1$ points wide. The cost to evaluate the approximation in $d$ space dimensions is proportional to $(p+1)$ operations (or memory accesses) on a Cartesian grid, and proportional to $(p+1)^{d}$ on a structured curvilinear grid (since the stencil is no-longer sparse). The curvilinear grid approximation also requires more memory per grid point to store grid metric information, and accessing these metric terms has an impact on the CPU time. The curvilinear grid can thus be expected to cost at least a factor of $(p+1)^{d-1}$ more than the Cartesian grid (which, for a second-order accurate scheme in three dimensions, is a factor of 9). We note that an unstructured grid approximation would likely require at least as many operations and memory accesses, and at least as much storage, as a curvilinear grid approximation. Thus, there appears to be a significant advantage in computational cost and efficiency of the method discussed here as compared to an unstructured grid method. Ease of grid generation is also an important factor to consider when comparing approaches and we note that, in general, construction of an overlapping grid for a complex geometry is currently more difficult than generating an unstructured tetrahedral mesh, but easier than generating a block-structured or hexahedral mesh. We recognize that higher-order accurate methods have clear advantages for many wave propagation problems ${ }^{6}$, but at present we focus on second-order accurate schemes and leave an investigation of higher-order methods to future work. Also, the numerical approach presented here for linear elasticity with constant material parameters on overlapping grids is considered to be a first step towards efficient finite difference and finite volume methods for variable material parameters, nonlinear models of solid mechanics and, more generally, for multi-physics models involving fluid-structure interactions [6].

An overlapping (overset, Chimera) grid consists of a collection of structured component grids that cover a domain of interest and overlap where they meet [7]. Solution values on different component grids are matched by interpolation at overlapping boundaries. Overlapping grids can be used to develop efficient methods through the use of structured grids and Cartesian grids. Overlapping grids have been used to solve partial differential equations for a wide range of problems. They have primarily been used for applications in fluid dynamics (see $[8,9,10,11,12]$ and the references therein), but more recently they have been used in other areas, including electromagnetics [5] and conjugate heat transfer [13], among others.

Despite the use of overlapping grids in other areas (many of which are governed by hyperbolic partial differential equations) there has been relatively little use of the approach for problems in elastodynamics. The first use of overlapping grids for problems of elastodynamics (to our knowledge) was described by Lin and Ballmann [14] and Niethammer, Kim and Ballmann [15]. In these papers the governing equations are solved as a first-order system for velocity and stress. Graphical comparisons to known solutions and experiments are made, but no convergence results are given in the paper and the important issue of stability for discretizations on overlapping grids is not addressed. In a more recent paper by Appelö, Nilsson, Petersson

\footnotetext{
${ }^{5}$ The computer code is called cgsm and is built on the Overture framework for overlapping grids. The software is freely available from the Overture web site www.llnl.gov/casc/Overture.

${ }^{6}$ Indeed the performance benefit of high-order finite difference methods on Cartesian grids relative to curvilinear grids is even greater than for second-order methods: the cost per grid point of the three-dimensional fourth-accurate Cartesian-grid scheme for Maxwell's equations [5] is approximately 25 times faster than the curvilinear grid version. Note that this factor of 25 agrees with the estimate $(p+1)^{d-1}$, for this case when $p=4$ and $d=3$.
} 
and Sjögreen [16] the governing equations are solved as a second-order system and results are presented for one overlapping grid computation, but again no convergence results are given. These papers, the earlier work in $[15,14]$ and the more recent work in [16], appear to be the only ones that consider numerical methods for elastodynamics on overlapping grids. We thus see a need for a careful investigation of schemes for the equations of elastodynamics on overlapping grids in order to assess the accuracy (convergence rates as the mesh is refined), stability in the presence of overlapping grid boundaries, solution behavior as a function of the material parameters, and computational performance. It is a principal aim of the present paper to address these issues for two different schemes, one based on the equations written as a first-order system and the other based on the second-order form of the equations; these being the two most popular classes of methods. This is accomplished by performing a stability analysis of the schemes and computing numerical solutions for a carefully chosen set of problems which illustrate the results of the analysis and describe the comparative accuracy, solution behavior, and performance of the two methods.

The numerical scheme for the second-order system, denoted by SOS, uses a conservative finite-difference approximation of the equations for the components of displacement. On a single curvilinear grid the SOS scheme is stable, non-dissipative and energy preserving. The non-dissipative nature of the scheme is an attractive property for wave propagation problems, but this also means that small changes to the numerical approximation could result in an unstable scheme. It is found, for example, that the interpolation equations needed for overlapping grids tend to excite an instability in the SOS scheme. This instability may be weak or strong depending on the configuration of the component grids in the overlapping grid. For example, when narrow curvilinear grids are used near physical boundaries, an unstable mode generated from the interpolation equations may be amplified due to interactions with the nearby boundary. This instability is stronger than the one created from an isolated interpolation boundary. A normal-mode analysis is performed for the one-dimensional wave equation in second-order form to illustrate this instability. We show that adding an explicit high-order dissipation term to the equations and/or using a separate high-order, low-pass filter step can be used to stabilize the SOS scheme for overlapping grids.

The numerical scheme for the first-order system, denoted by FOS, uses a second-order finite-volume (Godunov) approximation of the equations for the components of displacement, velocity and stress. In a somewhat novel approach, we evolve the displacements together with the velocity and stress. All components are coupled through the boundary conditions which helps to maintain consistency in the stress-strain relationship. The characteristic-based upwind FOS scheme is stable on a single curvilinear grid, and has some inherent high-order dissipation. A normal-mode stability analysis for a centered, non-dissipative (nonupwinded) FOS scheme applied to a one-dimensional model problem shows that it would be unstable (similar to that for the SOS scheme). However, the dissipation built into the upwind Godunov FOS scheme is found to be sufficient to stabilize the method for overlapping grids. The stability results for both the SOS and FOS schemes are obtained analytically for model problems involving scalar wave equations. Numerical results are then used to confirm the results of the analysis for the full equations of linear elasticity.

We note that the stability of hyperbolic problems on overlapping grids has been considered previously by various authors. The case of adaptive mesh refinement, a special case of an overlapping grid, has been considered by Browning, Kreiss and Oliger [17] and Ciment [18], for example. Berger [19] considered the stability of the first-order wave equation with mesh refinement in space and time. Starius [20] studied the stability of the Lax-Wendroff scheme for the first-order wave equation on a semi-infinite one-dimensional overlapping grid. Reyna [21] also analyzed the first-order wave equation on an overlapping grid and showed that the leap-frog scheme could be unstable. Pärt-Enander and Sjögreen [22] considered the stability of conservative interpolation on overlapping grids. Olsson and Petersson [23] studied the first-order wave equation and showed the existence of a class of unstable modes. They also demonstrated numerically that the growth rates of the unstable modes increased as the mesh was refined, although quite slowly. In the present paper, we extend these previous results for overlapping grids by considering the new situation of the second-order wave equation and also present new results for the first-order wave equation (the wave equation being used as a model for the equations of linear elasticity). In particular, we analyze the case of a one-dimensional overlapping grid when the number of grid points on one grid remains fixed as the mesh is refined. We show that the growth rate of unstable modes increases in proportion to $1 / h$, where $h$ is the mesh size of the grid. This important new result, which is applicable to the solution of any type of wave propagation problem on overlapping grids, dictates the form of dissipation that is required to stabilize the scheme. We describe a fourth-order dissipation and a high-order filter and show how they can be effective in suppressing such instabilities for the case of the SOS scheme. For the FOS approach, we show that the high-order dissipation built in to the Godunov scheme naturally provides dissipation of the appropriate form, and is sufficient to suppress the instabilities (although we do not prove this). The stability of the two schemes 
is considered for the case when the ratio $\lambda / \mu$ of the Lamé parameters is order one and for the case when the ratio becomes large. The latter case is well known to be a difficult regime near traction (stress-free) boundaries [24].

We illustrate the accuracy and stability of the SOS and FOS schemes for a wide range of initial-boundaryvalue problems in both two and three space dimensions. These problems demonstrate the behavior of the two schemes compared to one another and additionally serve as useful benchmarks for time-dependent elasticity. For some of these problems, we also employ a block-structured AMR approach to locally increase resolution. Our AMR scheme is based on the methodology originally developed by Berger and Oliger [25] for hyperbolic equations, and extended to overlapping grid following our early work in [8]. In this approach, a hierarchy of refinement grids is constructed dynamically based on a suitable error estimate of the solution. We also make use of a parallel implementation of our schemes for overlapping grids following the approach described in our recent work in [10]. Finally, the comparative performance of the two schemes is discussed for a representative problem in three space dimensions.

The remaining sections of the paper are organized as follows. In Section 2, we present the governing equations and boundary conditions for both the second-order and first-order systems. A brief description of the overlapping-grid approach, as well as the application of AMR and distributed parallel computing for overlapping grids, is provided in Section 3. The SOS and FOS schemes are described in Section 4. The stability of the SOS and FOS schemes for model wave equations on overlapping grids is considered in Section 5. A variety of test problems are considered in Section 6, and these are used to verify and compare the accuracy and performance of the two schemes, and to show that the schemes are stable for overlapping grids. Section 7 provides a discussion of the accuracy of the schemes as a function of points per wavelength. Concluding remarks are given in Section 8.

\section{Governing equations}

Consider an elastic solid that at time $t=0$ occupies the domain $\Omega \subset \mathbb{R}^{n_{d}}$ in $n_{d}=2$ or $n_{d}=3$ space dimensions. Let $\mathbf{u}(\mathbf{x}, t)$, with components $u_{i}(\mathbf{x}, t)$, denote the displacement of a material particle originally located at position $\mathbf{x} \in \mathbb{R}^{n_{d}}$, and let $\boldsymbol{\sigma}(\mathbf{x}, t)$ denote the Cauchy stress tensor with components $\sigma_{i j}(\mathbf{x}, t)$. It is assumed that the solid is a homogeneous isotropic material, and that the evolution of the displacement is governed by the equations of linear elasticity given by (with Einstein summation convention),

$$
\rho \frac{\partial^{2} u_{i}}{\partial t^{2}}=\frac{\partial \sigma_{i j}}{\partial x_{j}}+\rho f_{i}, \quad \mathbf{x} \in \Omega, \quad t>0, \quad i=1,2, \ldots, n_{d},
$$

where $\rho$ is the density of the material (taken to be constant), $\mathbf{f}$ is an acceleration due to an applied body force, and the components of stress are given by

$$
\sigma_{i j}=\lambda\left(\epsilon_{k k}\right) \delta_{i j}+2 \mu \epsilon_{i j}, \quad \epsilon_{i j}=\frac{1}{2}\left(\frac{\partial u_{i}}{\partial x_{j}}+\frac{\partial u_{j}}{\partial x_{i}}\right) .
$$

Here, $\epsilon_{i j}$ and $\delta_{i j}$ are the components of the (linear) strain tensor and the identity tensor, respectively, $\epsilon_{k k}=\sum_{k} \epsilon_{k k}=\nabla \cdot \mathbf{u}$ is the divergence of the displacement, and $\lambda$ and $\mu$ are Lamé parameters. The latter are related to Young's modulus $E$ and Poisson's ratio $\nu$ by $\mu=E /(2(1+\nu))$, and $\lambda=\nu E /((1+\nu)(1-2 \nu))$. Initial conditions for the second-order system in (1) are

$$
\mathbf{u}(\mathbf{x}, 0)=\mathbf{u}_{0}(\mathbf{x}), \quad \frac{\partial \mathbf{u}}{\partial t}(\mathbf{x}, 0)=\mathbf{v}_{0}(\mathbf{x}), \quad \mathbf{x} \in \Omega,
$$

where $\mathbf{u}_{0}(\mathbf{x})$ and $\mathbf{v}_{0}(\mathbf{x})$ are the initial displacement and velocity of the solid, respectively. Boundary conditions for (1) are applied for $\mathbf{x} \in \partial \Omega$ and take various forms. The boundary conditions considered in this paper are

$$
\left.\begin{array}{ll}
\mathbf{u}=\mathbf{g}_{d}(\mathbf{x}, t), & \text { displacement boundary condition, } \\
\mathbf{n} \cdot \boldsymbol{\sigma}=\mathbf{g}_{t}(\mathbf{x}, t), & \text { traction boundary condition } \\
\mathbf{n} \cdot \mathbf{u}=g_{s}(\mathbf{x}, t) & \\
\mathbf{n} \cdot \sigma \cdot \boldsymbol{\tau}_{\alpha}=g_{s, \alpha}(\mathbf{x}, t)
\end{array}\right\} \quad \begin{aligned}
& \text { slip-wall boundary conditions. }
\end{aligned}
$$

Here, $\mathbf{n}$ is the unit outward normal on the boundary and $\boldsymbol{\tau}_{\alpha}, \alpha=1, \ldots, n_{d}-1$, are unit tangent vectors (assumed to be mutually orthogonal). The functions $\mathbf{g}_{d}(\mathbf{x}, t)$ and $\mathbf{g}_{t}(\mathbf{x}, t)$ give the displacement and traction 
at the boundary, respectively, while $g_{s}(\mathbf{x}, t)$ and $g_{s, \alpha}(\mathbf{x}, t)$ define the slip wall motion. The elastic wave equation (1)-(2) with initial conditions (3) and boundary conditions (4)-(6) is a well-posed problem, see, for example [26].

We also consider the equations in (1) and (2) written as a first-order system

$$
\left.\begin{array}{l}
\frac{\partial u_{i}}{\partial t}=v_{i}, \\
\frac{\partial v_{i}}{\partial t}=\frac{1}{\rho} \frac{\partial \sigma_{i j}}{\partial x_{j}}+f_{i}, \\
\frac{\partial \sigma_{i j}}{\partial t}=\lambda\left(\dot{\epsilon}_{k k}\right) \delta_{i j}+2 \mu \dot{\epsilon}_{i j},
\end{array}\right\} \quad \mathbf{x} \in \Omega, \quad t>0, i=1,2, \ldots, n_{d}
$$

where $\mathbf{v}(\mathbf{x}, t)$, with components $v_{i}(\mathbf{x}, t)$, is the velocity and the components $\dot{\epsilon}_{i j}$ of the rate of strain tensor are given by

$$
\dot{\epsilon}_{i j}=\frac{1}{2}\left(\frac{\partial v_{i}}{\partial x_{j}}+\frac{\partial v_{j}}{\partial x_{i}}\right) .
$$

Initial conditions for displacement and velocity are given by $\mathbf{u}_{0}(\mathbf{x})$ and $\mathbf{v}_{0}(\mathbf{x})$ as before, and initial conditions for the components of stress may be derived from (2) applied at $t=0$. Boundary conditions for the first-order system may be taken directly from those described in (4), (5) and (6) for the second-order system. Note that contrary to what is typically done, we retain the displacements in our formulation of the first order system. The displacements are coupled with the velocity and stress through the boundary conditions as discussed in Appendix A.2. Retaining the displacements in the formulation allows the stress-strain relationship (2) to be explicitly imposed at the boundary. In addition it will be useful to have the displacement field when solving fluid-structure interaction problems (to define the fluid-solid interface for grid generation, for example).

The governing equations, whether written as a second-order or first-order system, are hyperbolic and represent the motion of elastic waves in the solid. For the second-order system, the characteristic wave speeds for a homogeneous material in a periodic or infinite space are $\pm c_{p}$ and $\pm c_{s}$, where the pressure and shear wave speeds are given by

$$
c_{p}=\sqrt{\frac{\lambda+2 \mu}{\rho}}, \quad c_{s}=\sqrt{\frac{\mu}{\rho}} .
$$

The first-order system has the wave speeds above as well as characteristics speeds equal to zero.

\section{Overlapping grid framework}

An overlapping grid, $\mathcal{G}$, consists of a set of structured component grids, $\left\{G_{g}\right\}, g=1, \ldots, \mathcal{N}$, that cover the domain $\Omega$ and overlap where the component grids meet. Typically, boundary-fitted curvilinear grids are used near the boundaries while one or more background Cartesian grids are used to handle the bulk of the domain. Each component grid is a logically rectangular, curvilinear grid in $n_{d}$ space dimensions $\left(n_{d}=2\right.$ or 3), and is defined by a smooth mapping from parameter space $\mathbf{r}$ (the unit square or cube) to physical space $\mathbf{x}$,

$$
\mathbf{x}=\mathbf{G}_{g}(\mathbf{r}), \quad \mathbf{r} \in[0,1]^{n_{d}}, \quad \mathbf{x} \in \mathbb{R}^{n_{d}} .
$$

If a calculation is performed using adaptive mesh refinement (AMR), then this mapping (belonging to a component grid at the base level) is used to define the location of grid points at any desired resolution for its refinement grids, and to define the associated mappings for these refinement grids. In the subsections below, we first give an example of an overlapping grid (at the base-level of refinement) and then we provide a brief discussion of AMR on overlapping grids. We conclude this section with a brief discussion of our parallel approach for overlapping grids.

\subsection{Base-level overlapping grid}

At the base level, an overlapping grids consists of a set of component grids that cover a domain $\Omega$. As an illustration, Figure 1 shows a simple overlapping grid consisting of two component grids, an annular boundary-fitted grid and a background Cartesian grid. The top view shows the overlapping grid while the bottom view shows each grid in parameter space. In this example the annular grid cuts a hole in the Cartesian grid so that the latter grid has a number of unused points which are marked as open circles. The other points on the component grids are marked as discretization points (where the PDE or boundary 

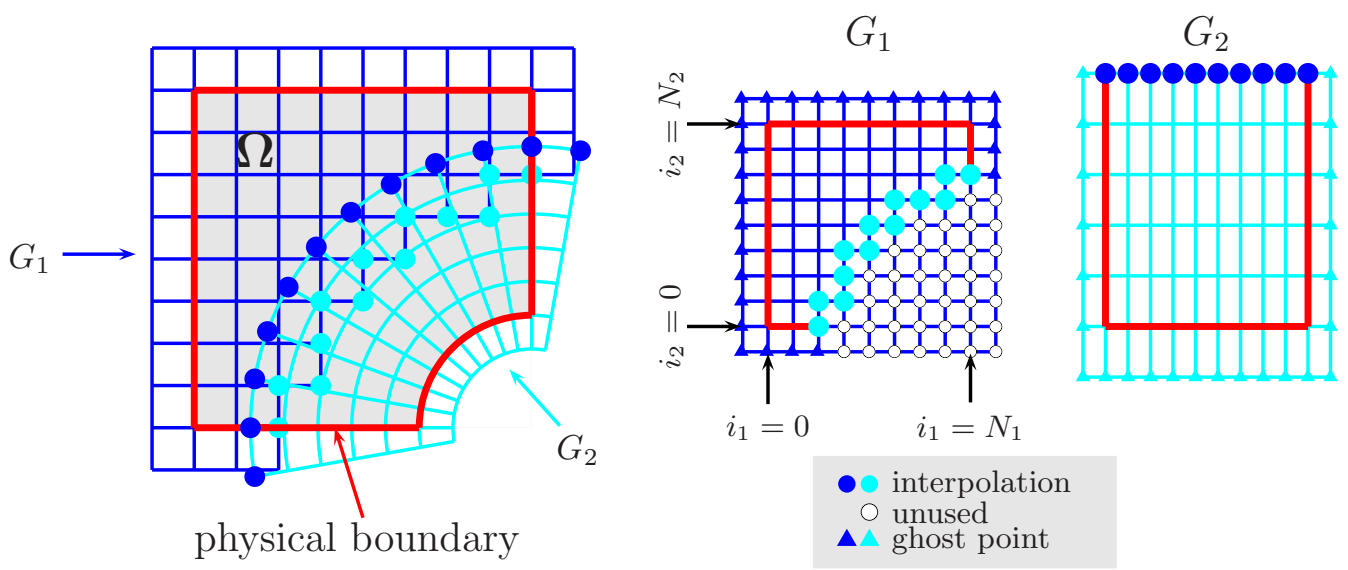

Figure 1: Left: an overlapping grid consisting of two structured curvilinear component grids. Middle and right: component grids for the square and annular grids in the unit square parameter space. Grid points are classified as discretization points, interpolation points or unused points. Ghost points are used to apply boundary conditions.

conditions are discretized) and interpolation points. Solution values at interpolation points are generally determined by a tensor-product Lagrange interpolant in the parameter space of the donor grid. Ghost points are used to facilitate the discretization of boundary conditions.

The classification of grid points into discretization, interpolation and unused points is determined by an overlapping grid generator. We use the Ogen grid generator [27]. Ogen takes as input a set of overlapping (base-level) component grids along with a classification of the boundaries of each grid as a physical boundary, an interpolation boundary or a periodic boundary. Unused points are determined by Ogen using physical boundaries to mark points exterior to the domain following a hole-cutting algorithm. The remaining points are classified as either discretization points or interpolation points.

\subsection{Adaptive mesh refinement}

Adaptive mesh refinement is a well-known numerical approach to solve PDEs (often hyperbolic) efficiently. The approach used here, which follows that described in [8] and [10], is designed to locally increase the grid resolution where an estimate of the error is large. For an overlapping grid, this is done by adding refined grid patches to the existing base-level component grids as mentioned above. The refinement grids are aligned with the underlying base grid (i.e. the refinement is done in parameter space) and are arranged in a hierarchy with the base grids belonging to level $\ell=0$, the next finer grids being added to level $\ell=1$ and so on. Grids on level $\ell$ are refined by a refinement ratio $n_{r}$ from the grids on level $\ell-1$. The grids are properly nested so that a grid on level $\ell$ is completely contained in the set of grids on the coarser level $\ell-1$. This requirement is relaxed at physical boundaries to allow refinement grids to align with the boundary.

The numerical solution on all grids is advanced in time using the same global time step. For an AMR calculation the main computational cost usually involves advancing the solution on the finest grid level. Thus, the use of an artificially small time step to advance the solution on coarser grids does not have a large impact on the overall efficiency of the time stepping. The use of a global time step is simpler, but it is possible to advance the solution on each grid with the optimal time step for that grid, and use interpolation in time at the overlap and at AMR boundaries; we leave this as a future optimization. After every $n_{\text {regrid }}$ time steps, the whole refined-grid hierarchy is rebuilt to accommodate the evolution of sharp features of the solution (such pressure or shear waves in elasticity). This is done by first re-computing an estimate of the error given by

$$
e_{\mathbf{i}}=\sum_{k=1}^{n_{c}} e_{k, \mathbf{i}}
$$

where the error is estimated as a sum of error estimates for each component,

$$
e_{k, \mathbf{i}}=\frac{1}{n_{d}} \sum_{j=1}^{n_{d}}\left(\frac{c_{1}}{s_{k}}\left|\Delta_{0 j} w_{k, \mathbf{i}}\right|+\frac{c_{2}}{s_{k}}\left|\Delta_{+j} \Delta_{-j} w_{k, \mathbf{i}}\right|\right) .
$$

In (10), $w_{k, \mathbf{i}}$ is the $k$ th component of the numerical solution at grid index $\mathbf{i}, s_{k}$ is a scale factor for component $k$, and $c_{1}$ and $c_{2}$ are weights for the scaled approximations to the first and second derivatives of the solution in 
parameter space. The operators $\Delta_{0 j}, \Delta_{+j}$ and $\Delta_{-j}$ are the usual centered, forward and backward undivided difference operators in the $j$ index direction, respectively. For example,

$$
\begin{aligned}
\Delta_{01} w_{k, \mathbf{i}}^{n} & =\left(w_{k, i_{1}+1, i_{2}, i_{3}}-w_{k, i_{1}-1, i_{2}, i_{3}}\right) / 2, \\
\Delta_{+2} w_{k, \mathbf{i}}^{n} & =\left(w_{k, i_{1}, i_{2}+1, i_{3}}-w_{k, \mathbf{i}}\right), \\
\Delta_{-3} w_{k, \mathbf{i}}^{n} & =\left(w_{k, \mathbf{i}}-w_{k, i_{1}, i_{2}, i_{3}-1}\right), \\
\Delta_{+2} \Delta_{-2} w_{k, \mathbf{i}}^{n} & =\left(w_{k, i_{1}, i_{2}+1, i_{3}}-2 w_{k, \mathbf{i}}+w_{k, i_{1}, i_{2}-1, i_{3}}\right) .
\end{aligned}
$$

For the discrete approximation of the second-order system, $w_{k, \mathbf{i}}$ represents the components of displacement, while for the approximation of the first-order system, the components of velocity and stress are also included. The error estimate used here follows that introduced in [8] which was found to be an effective choice, although other methods are possible. Once the error estimate is computed, it is smoothed and then grid points are tagged for refinement where $e_{\mathbf{i}}$ is greater than a chosen tolerance. Buffer points are added to increase the region of tagged points slightly (so that fewer regrids are needed), and a new overlapping grid hierarchy is build to cover the buffered region of tagged points. The numerical solution at the current time $t$ is then transferred from the old grid hierarchy to the new one, and the time-stepping proceeds for the solution on the new grid hierarchy until the next gridding step. The re-gridding procedure described here applies to any two-level time marching scheme, such as the one for the first-order system (see Section 4.2). For the case of the second-order system, the numerical scheme involves three time levels (see Section 4.1), and we accommodate this by transferring (interpolating) the solutions at the current time $t$ and at the previous time $t-\Delta t$ from the old grid hierarchy to the new one during each re-grid step.

\subsection{Parallel}

For parallel computations we use a domain decomposition approach. The component grids of the overlapping grid, including all base-level and refinement grids, are partitioned across a set of processors. Each grid, and associated grid functions, are partitioned in a tensor product fashion over a contiguous range of processors (the latter requirement being a restriction of the version of the Multiblock PARTI parallel communication library [28] that we use). A load-balancing algorithm determines how to partition the grids on a distributed memory machine (e.g. which processors to use for each grid). We currently use a modified bin-packing algorithm for loading balancing. Parallel communication is performed using the Message Passing Interface, MPI [29]. All aspects of the algorithm including overlapping grid interpolation, AMR interpolation and regridding are performed in parallel. Section 6.8 presents some results from the parallel version of the code. A full discussion of our parallel approach, including scaling results, is given in [10].

\section{Discretization of the governing equations}

The discretization of the governing equations is carried out on a uniform grid in the unit (parameter) computational space $\mathbf{r} \in[0,1]^{n_{d}}$. The mapping $\mathbf{x}=\mathbf{G}_{g}(\mathbf{r})$ defines the grid in physical space $\mathbf{x}$, and an exact changes of variables is made to express the governing equations in computational space. A discretization is then performed on the mapped equations. This is described for the second and first-order systems in the two subsections below. A discussion of the discretization of the boundary conditions is given in Appendix A.

\subsection{Second-order system}

The mapped equations corresponding to the second-order system in (1) and (2) may be written in the conservation form

$$
\begin{aligned}
\rho \frac{\partial^{2} u_{i}}{\partial t^{2}} & =\frac{1}{J} \frac{\partial}{\partial r_{j}}\left(J \frac{\partial r_{j}}{\partial x_{k}} \sigma_{k i}\right)+\rho f_{i}, \\
\sigma_{i j} & =\lambda \epsilon_{k k} \delta_{i j}+2 \mu \epsilon_{i j}, \\
\epsilon_{i j} & =\frac{1}{2}\left(\frac{\partial r_{k}}{\partial x_{j}} \frac{\partial u_{i}}{\partial r_{k}}+\frac{\partial r_{k}}{\partial x_{i}} \frac{\partial u_{j}}{\partial r_{k}}\right),
\end{aligned}
$$

where $J=\operatorname{det}(\partial \mathbf{x} / \partial \mathbf{r})$ is the Jacobian of the mapping $\mathbf{x}=\mathbf{G}_{g}(\mathbf{r})$. These equations can be written succinctly as

$$
\rho \frac{\partial^{2} u_{i}}{\partial t^{2}}=\frac{1}{J} \frac{\partial}{\partial r_{j}}\left(A_{i j k l} \frac{\partial u_{k}}{\partial r_{l}}\right)+\rho f_{i}
$$


Expressions for the coefficients $A_{i j k l}$ can be obtained by substituting (14) and (13) into (12). The equations in (15) can be discretized to second-order accuracy using a compact stencil (of width equal to three in each direction). The terms on the right-hand side of (15) involve unmixed and mixed second-order derivatives, and these are approximated using

$$
\begin{aligned}
& \left.\frac{\partial}{\partial r_{j}}\left(a(\mathbf{x}) \frac{\partial u_{k}}{\partial r_{j}}\right)\right|_{\mathbf{x}_{\mathbf{i}}, t_{n}} \approx D_{+j}\left(\left(\mathcal{A}_{-j} a\left(\mathbf{x}_{\mathbf{i}}\right) D_{-j} u_{k, \mathbf{i}}^{n}\right),\right. \\
& \left.\frac{\partial}{\partial r_{j}}\left(a(\mathbf{x}) \frac{\partial u_{k}}{\partial r_{l}}\right)\right|_{\mathbf{x}_{\mathbf{i}}, t_{n}} \approx D_{0 j}\left(a\left(\mathbf{x}_{\mathbf{i}}\right) D_{0 l} u_{k, \mathbf{i}}^{n}\right), \quad j \neq l,
\end{aligned}
$$

respectively, where $u_{k, \mathbf{i}}^{n} \approx u_{k}\left(\mathbf{x}_{\mathbf{i}}, t_{n}\right)$ and $a(\mathbf{x})$ is a component of $A_{i j k l}$, and where $D_{+j}, D_{-j}$ and $D_{0 j}$ are the usual forward, backward and central (divided) difference approximations in the $j^{\text {th }}$ coordinate direction and $\mathcal{A}_{-j}$ is the averaging operator. For example,

$$
\begin{aligned}
& D_{+1} u_{k, \mathbf{i}}^{n}=\left(u_{k, i_{1}+1, i_{2}, i_{3}}^{n}-u_{k, \mathbf{i}}^{n}\right) / \Delta r_{1}, \\
& D_{-2} u_{k, \mathbf{i}}^{n}=\left(u_{k, \mathbf{i}}^{n}-u_{k, i_{1}, i_{2}-1, i_{3}}^{n}\right) / \Delta r_{2}, \\
& \mathcal{A}_{-3} u_{k, \mathbf{i}}^{n}=\left(u_{k, \mathbf{i}}^{n}+u_{k, i_{1}, i_{2}, i_{3}-1}^{n}\right) / 2 .
\end{aligned}
$$

For second-order accuracy we discretize the equations in time using a centered approximation

$$
\left.\frac{\partial^{2} u_{k}}{\partial t^{2}}\right|_{\mathbf{x}_{\mathbf{i}}, t_{n}} \approx \frac{u_{k, \mathbf{i}}^{n+1}-2 u_{k, \mathbf{i}}^{n}+u_{k, \mathbf{i}}^{n-1}}{\Delta t^{2}},
$$

and evaluate the forcing term as $f_{k, \mathbf{i}}^{n}=f_{k}\left(\mathbf{x}_{\mathbf{i}}, t_{n}\right)$. Using the finite-difference approximations in (16), (17) and (18), together with special one-sided approximations at traction boundaries, it can be shown that the overall scheme is stable, second-order accurate, non-dissipative and preserves a discrete approximation to the energy on a single curvilinear grid. (See Appelö and Petersson [30] for further details.)

The conservative scheme described above has no dissipation for a single mapped grid. On an overlapping grid, the interpolation equations required where grids overlap may introduce perturbations to the approximation that can cause instabilities. An analysis of the instability is given later in Section 5. For now, we describe two schemes to suppress this instability based on the addition of an artificial dissipation term or the application of a high-order low-pass filter.

The artificial dissipation term is added to the discrete equations corresponding to the right-hand side of (15), and it takes the form

$$
\mathcal{D}_{h}^{d} \mathbf{u}_{\mathbf{i}}^{n}=-\alpha_{d} \sum_{j=1}^{n_{d}}\left(-\Delta_{+j} \Delta_{-j}\right)^{d / 2}\left(\frac{\mathbf{u}_{\mathbf{i}}^{n}-\mathbf{u}_{\mathbf{i}}^{n-1}}{\Delta t}\right),
$$

where $d$ is the order of the dissipation (an even integer) and $\Delta_{ \pm j}$ are the undivided difference operators defined previously in (11). To avoid forming an implicit system, the dissipation (19) uses a backward difference in time. In Appendix B we show, for a model problem, that this approximation provides a stable way to add dissipation. For a second-order approximation we can use second-order dissipation $d=2$ or higher and still retain second-order accuracy overall. In many cases an $O(1)$ value for $\alpha_{d}$ (e.g. $\alpha_{d}=1$ ) will lead to a stable numerical solution. However, in more difficult cases involving certain ranges of the constitutive parameters and overlapping-grid configurations as is discussed in Section 5, it is necessary to increase the coefficient as the mesh is refined, $\alpha_{d} \propto 1 / h$, for example. In these cases one should use at least fourth-order dissipation to retain second-order accuracy overall. We have also found that the use of a high-order filter is generally a good choice for these difficult cases (see Section 5.1.2). The high-order low-pass filter is applied to the grid function $\mathbf{u}_{\mathbf{i}}^{n}$ to obtain a new filtered solution $\mathbf{u}^{n, *}$ as follows:

$$
\begin{aligned}
\mathbf{u}_{\mathbf{i}}^{n, *} & =\mathcal{F}_{d}\left(\mathbf{u}_{\mathbf{i}}^{n}\right), \\
\mathcal{F}_{d}\left(\mathbf{u}_{\mathbf{i}}^{n}\right) & =\mathbf{u}_{\mathbf{i}}^{n}-\beta_{d} \sum_{j=1}^{n_{d}}\left(-\Delta_{+j} \Delta_{-j}\right)^{d / 2} \mathbf{u}_{\mathbf{i}}^{n} .
\end{aligned}
$$

The filter can be applied every time step or every few time steps. The value of the coefficient $\beta_{d}$ is often taken to be $1 /\left(2^{d} n_{d}\right)$ so that the filter eliminates the most oscillatory grid function $\mathbf{u}_{\mathbf{i}}=(-1)^{i_{1}}(-1)^{i_{2}}(-1)^{i_{3}}$. This plus-minus component is often the most unstable mode. 
If the filter in $(21)$ is applied at every time step, then the error introduced is $O\left(h^{d} / \Delta t\right)$. Choosing a fourth-order filter, $d=4$, (or higher) retains the second-order accuracy of the scheme. We have found that a sixth-order filter, $d=6$, is a good choice since it is effective at stabilizing the scheme but results in little dissipation to the resolved modes of the computed solution. The fourth and sixth-order filters require special treatment near boundaries due to their wide stencils. Rather than develop special one-sided approximations (as done in [31] for example) we instead assign values of the solution at extra ghost points using extrapolation.

The Fourier transform of the one-dimensional filter (transfer-function) is shown in Figure 2 and given by

$$
\widehat{\mathcal{F}}_{d}(\xi)=1-\sin ^{d}(\xi / 2), \quad|\xi| \leq \pi
$$

where $\xi$ is the normalized wave number. It can be seen that the higher-order filters have little effect on the low frequency components of the solution while still strongly damping the high frequencies. We note that another good filter might be the compact filters proposed by Lele and others [32].

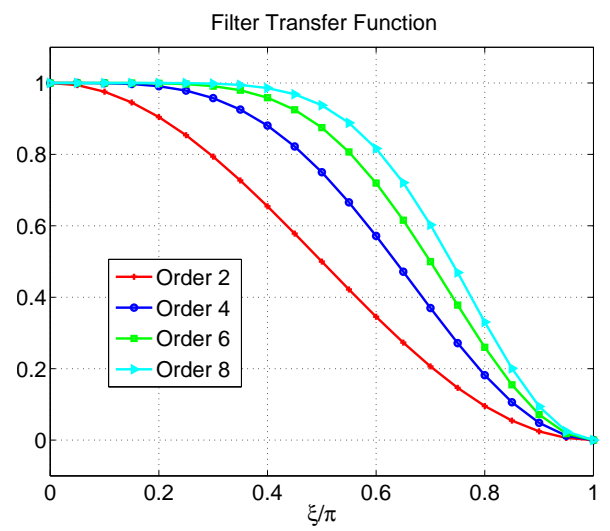

Figure 2: Transfer function for the high-order filter. The plus-minus wave, which corresponds to $\xi / \pi=1$, is completely damped by the filter.

On a Cartesian grid the time step for the SOS scheme (without dissipation) is determined as

$$
\Delta t=C_{\mathrm{FL}} \frac{1}{c_{p}}\left(\sum_{j=1}^{n_{d}} \frac{1}{\Delta x_{j}^{2}}\right)^{-1 / 2}
$$

where $c_{p}$ is the p-wave speed (8) and the parameter $C_{\mathrm{FL}}$ is usually taken to be 0.9 . The corresponding formula for curvilinear grids and the addition of dissipation is determined in the usual way by freezing coefficients and using a local Fourier analysis. Note that the overall time step for an overlapping grid is taken as the minimum value of $\Delta t$ computed for each component grid (including AMR grids).

\subsection{First-order system}

In this section, we consider a discretization of the first-order system of equations in (7). In Cartesian coordinates, the system of equations for velocity and stress can be written in the form

$$
\frac{\partial \mathbf{w}}{\partial t}+\sum_{\alpha=1}^{n_{d}} \frac{\partial}{\partial x_{\alpha}}\left(A^{(\alpha)} \mathbf{w}\right)=\mathbf{h}
$$

where $\mathbf{w}=[\mathbf{v}, \boldsymbol{\sigma}]^{T} \in \mathbb{R}^{n_{c}}$ is a vector of dimension $n_{c}=n_{d}+n_{d}^{2}$ containing the components of velocity and stress, $A^{(\alpha)}$ are coefficient matrices which depend on $\rho, \lambda$ and $\mu$, and $\mathbf{h}=\mathbf{h}(\mathbf{x}, t)$ denotes the forcing. In terms of the computational space coordinates $\mathbf{r}$, defined from a (known) smooth mapping $\mathbf{x}=\mathbf{G}(\mathbf{r})$, the system in (24) becomes

$$
\frac{\partial}{\partial t} \mathbf{w}+\frac{1}{J} \sum_{\alpha=1}^{n_{d}} \frac{\partial}{\partial r_{\alpha}} \mathbf{f}^{(\alpha)}(\mathbf{w})=\mathbf{h}
$$

where

$$
\mathbf{f}^{(\alpha)}(\mathbf{w})=J C_{\alpha} \mathbf{w}, \quad J=\operatorname{det}\left(\frac{\partial \mathbf{x}}{\partial \mathbf{r}}\right), \quad C_{\alpha}=\sum_{j=1}^{n_{d}} \frac{\partial r_{\alpha}}{\partial x_{j}} A^{(j)}
$$


The discretization of the mapped equations in (25) is performed on a uniform grid with mesh spacings $\Delta r_{\alpha}$ for $\alpha=1, \ldots, n_{d}$. We use the standard form

$$
\mathbf{w}_{\mathbf{i}}^{n+1}=\mathbf{w}_{\mathbf{i}}^{n}-\frac{\Delta t}{J_{\mathbf{i}}} \sum_{\alpha=1}^{n_{d}} D_{+\alpha} \mathbf{f}_{\mathbf{i}-\frac{1}{2} \hat{\mathbf{e}}_{\alpha}}^{(\alpha)}+\Delta t \mathbf{h}_{\mathbf{i}}^{n+1 / 2},
$$

where $\mathbf{w}_{\mathbf{i}}^{n}$ is an approximation of $\mathbf{w}\left(\mathbf{r}_{\mathbf{i}}, t_{n}\right), \mathbf{f}_{\mathbf{i}}^{(\alpha)}, \alpha=1, \ldots, n_{d}$ are numerical approximations of the fluxes defined in (26), $\mathbf{h}_{\mathbf{i}}^{n+1 / 2}$ is an approximation to the source term, and $\hat{\mathbf{e}}_{\alpha} \in \mathbb{R}^{n_{d}}$ is the unit vector in the $\alpha$-direction.

There are many possible choices for the numerical fluxes in (26). Our choice is based on the exact Godunov flux, which for the linear equations in (25) and (26) becomes

$$
\mathbf{f}_{\mathbf{i}+\frac{1}{2} \hat{\mathbf{e}}_{\alpha}}^{(\alpha)}=\frac{1}{2}\left(\mathbf{f}^{(\alpha)}\left(\mathbf{w}_{R}\right)+\mathbf{f}^{(\alpha)}\left(\mathbf{w}_{L}\right)\right)-E_{\alpha}^{\frac{1}{2}}\left[\frac{J_{\mathbf{i}}}{2} \sum_{m=1}^{n_{c}} \Gamma_{\alpha, \mathbf{i}}^{(m)}\left|K_{\alpha, \mathbf{i}}^{(m)}\right| \mathbf{z}_{\alpha, \mathbf{i}}^{(m)}\right],
$$

for left and right states given by $\mathbf{w}_{L}$ and $\mathbf{w}_{R}$, respectively. Here, $K_{\alpha}^{(m)}$ and $\mathbf{z}_{\alpha}^{(m)}, m=1,2, \ldots, n_{c}$, are the eigenvalues and eigenvectors belonging to the matrix $C_{\alpha}$ defined in (26), and $\Gamma_{\alpha}^{(m)}$ is the $m^{\text {th }}$ component of the vector $Z_{\alpha}^{-1}\left(\mathbf{w}_{R}-\mathbf{w}_{L}\right)$, where $Z_{\alpha}$ is the matrix of eigenvectors. The shift operator, $E_{\alpha}^{\frac{1}{2}}$, that appears in (28) is defined by $E_{\alpha}^{\beta} \mathbf{w}_{\mathbf{i}}=\mathbf{w}_{\mathbf{i}+\beta \hat{\mathbf{e}}_{\alpha}}$, and values defined on the faces, $\mathbf{i}+\frac{1}{2} \hat{\mathbf{e}}_{\alpha}$, are determined by averaging, e.g. $J_{\mathbf{i}+\frac{1}{2} \hat{\mathbf{e}}_{\alpha}}=\frac{1}{2}\left(J_{\mathbf{i}+\hat{\mathbf{e}}_{\alpha}}+J_{\mathbf{i}}\right)$.

The numerical flux defined in (28) with left and right states taken directly from grid values on either side of a given cell face is first-order accurate in general. Second-order accuracy can be achieved (for smooth solutions) if modified values for the left and right states are used. For example, consider the cell face with index $\mathbf{i}+\frac{1}{2} \hat{\mathbf{e}}_{\alpha}$ and define the (wave-strength) vector

$$
\mathbf{a}_{\alpha, \mathbf{i}}^{n}=\mathcal{L}\left(Z_{\alpha, \mathbf{i}}^{-1} \Delta_{-\alpha} \mathbf{w}_{\mathbf{i}}^{n}, Z_{\alpha, \mathbf{i}}^{-1} \Delta_{+\alpha} \mathbf{w}_{\mathbf{i}}^{n}\right)
$$

where $\mathcal{L}(\cdot, \cdot)$ is a slope-limiter function which is applied component-wise. For the Euler equations of gas dynamics a common choice is the minmod (minimum modulus) limiter, see our previous work in [8], for example. For the current work, however, we use an unlimited scheme with $\mathcal{L}(b, c)=(b+c) / 2$. Wave strengths defined in (29) are used to obtain the following values for the left and right states about the cell face $\mathbf{i}+\frac{1}{2} \hat{\mathbf{e}}_{\alpha}$ :

$$
\begin{gathered}
\mathbf{w}_{L}=\mathbf{w}_{\mathbf{i}}^{n}+\frac{1}{2} Z_{\alpha, \mathbf{i}} \mathbf{a}_{\alpha, \mathbf{i}}-\sum_{j=1}^{n_{d}} \frac{\Delta t}{2 \Delta r_{j}} Z_{j, \mathbf{i}} K_{j, \mathbf{i}} \mathbf{a}_{j, \mathbf{i}}^{n}+\frac{\Delta t}{2} \mathbf{h}_{\mathbf{i}}^{n}, \\
\mathbf{w}_{R}=E_{\alpha}\left[\mathbf{w}_{\mathbf{i}}^{n}-\frac{1}{2} Z_{\alpha, \mathbf{i}} \mathbf{a}_{\alpha, \mathbf{i}}-\sum_{j=1}^{n_{d}} \frac{\Delta t}{2 \Delta r_{j}} Z_{j, \mathbf{i}} K_{j, \mathbf{i}} \mathbf{a}_{j, \mathbf{i}}^{n}+\frac{\Delta t}{2} \mathbf{h}_{\mathbf{i}}^{n}\right] .
\end{gathered}
$$

The left and right states in (30) are used to compute $\mathbf{f}_{\mathbf{i}+\frac{1}{2} \hat{\mathbf{e}}_{\alpha}}^{(\alpha)}$, and similar formulas give left and right states about the other cell faces which may be used to compute the remaining numerical fluxes in (27).

We note that for the case of a Cartesian grid, the computation of the Godunov flux in (28) using the left and right states in (30) simplifies significantly in terms of numerical operations and memory accesses. Since the majority of grid points belong to Cartesian grids for a typical overlapping grid, we find it worthwhile to implement an optimized version of the FOS scheme for Cartesian grids that is significantly faster than the version for general curvilinear grids. Finally, we use the formula

$$
\mathbf{w}_{\mathbf{i}}^{n+1 / 2}=\mathbf{w}_{\mathbf{i}}^{n}-\sum_{j=1}^{n_{d}} \frac{\Delta t}{2 \Delta r_{j}} Z_{j, \mathbf{i}} K_{j, \mathbf{i}} \mathbf{a}_{j, \mathbf{i}}^{n}+\frac{\Delta t}{2} \mathbf{h}_{\mathbf{i}}^{n} .
$$

to obtain an approximation for $\mathbf{w}=[\mathbf{v}, \boldsymbol{\sigma}]^{T}$ at the cell center $\mathbf{r}_{\mathbf{i}}$ at time $t_{n}+\Delta t / 2$. The displacement $\mathbf{u}$ is then advanced according to

$$
\mathbf{u}_{\mathbf{i}}^{n+1}=\mathbf{u}_{\mathbf{i}}^{n}+\Delta t \mathbf{v}_{\mathbf{i}}^{n+1 / 2}
$$

where $\mathbf{v}_{\mathbf{i}}^{n+1 / 2}$ is the velocity belonging to $\mathbf{w}_{\mathbf{i}}^{n+1 / 2}$. 
On a Cartesian grid, the time step for the FOS scheme is determined by

$$
\Delta t=C_{\mathrm{FL}} \frac{1}{c_{p}}\left(\sum_{j=1}^{n_{d}} \frac{1}{\Delta x_{j}}\right)^{-1}
$$

where the parameter $C_{\mathrm{FL}}$ is usually taken to be 0.9 . Note that on a Cartesian grid with equal grid spacings in each coordinate direction, the time step for the SOS scheme from equation (33) is $\sqrt{n_{d}}$ times bigger than that for the FOS scheme.

\section{Stability at overlapping grid interfaces with nearby boundaries}

In this section we analyze some aspects of the stability of our schemes on overlapping grids. The two schemes discussed previously are stable for a single curvilinear grid assuming the time step is chosen to satisfy a CFL stability constraint. However, a numerical scheme that is stable on a single grid may become unstable on an overlapping grid, especially for the case of neutrally stable schemes (with no dissipation). In practice we often use a narrow curvilinear grid next to a boundary with a fixed number of grids points $N$ in the direction normal to the boundary. This is done so that as the overlapping grid is refined, the total number of grid points belonging to boundary-fitted grids decreases relative to the number of the grid points belonging to (more efficient) Cartesian grids used for the interior of the problem domain. Another choice for an overlapping grid would be to use boundary-fitted grids with fixed normal distance from the boundaries. This latter approach is more expensive computationally but less difficult from the point of view of stability as we show in the analysis below.

The equations of linear elasticity are a coupled set of wave equations. As a model problem we consider the solution to a scalar wave equation in one space dimension (corresponding to the direction normal to a boundary). We study discrete approximations of the wave equation in both second-order and first-order forms for an overlapping grid. We show that non-dissipative centered approximations are unstable for certain values of the overlap parameters (e.g. interpolation coefficients) and that the growth rates of these unstable modes increase as the mesh is refined. The centered schemes can be stabilized with artificial dissipation provided the coefficient of the artificial dissipation also increases as the mesh is refined. The Godunov scheme for the first-order wave equation has been found in practice to have enough inherent dissipation to be stable without requiring any added dissipation.

\subsection{Stability of the second-order wave equation}

Let us consider the second-order scalar wave equation in one space dimension for $u(x, t)$ defined on the semi-infinite interval $\Omega=(-\infty, b]$ for $t \geq 0$ :

$$
\begin{aligned}
\frac{\partial^{2} u}{\partial t^{2}} & =\frac{\partial^{2} u}{\partial x^{2}}, & & x \in(-\infty, b), t>0, \\
u(x, 0) & =u_{0}(x), \quad \frac{\partial u}{\partial t}(x, 0)=v_{0}(x), & & x \in(-\infty, b), \\
u(b, t) & =g(t), \quad|u(\cdot, t)|<\infty, & & t>0 .
\end{aligned}
$$

Here, the initial and boundary conditions are assumed to be consistent so that $u_{0}(b)=g(0)$ and $v_{0}(b)=$ $g^{\prime}(0)$, and we look for solutions that remain bounded as $x \rightarrow-\infty$. We solve this initial-boundary-value problem on an overlapping grid as shown in Figure 3. The solution is approximated by the grid functions

$u_{j}^{(m)}(t) \approx u\left(x_{j}^{(m)}, t\right), m=1,2$, on their component grids $x_{j}^{(m)}=x_{a}^{(m)}+j h_{m}$. The grid spacings are $h_{1}$ and $h_{2}$, assumed positive, and let $h=\min \left(h_{1}, h_{2}\right)$. This overlapping grid is representative of the grids we generally use where there is a narrow boundary-fitted grid with a fixed number of grid points in the direction normal to the boundary overlapping with a large background grid. The red grid in the figure with $m=1$ models the boundary-fitted grid and has $N=$ constant as the mesh is refined. The blue grid in the figure with $m=2$ represents the background grid (and is of infinite extent on the scale of the width of the boundary-fitted grid). Values for the interpolated end points $u_{0}^{(1)}$ and $u_{q}^{(2)}$ are obtained from values on grids $m=2$ and $m=1$, respectively, using interpolation as indicated in the figure. 


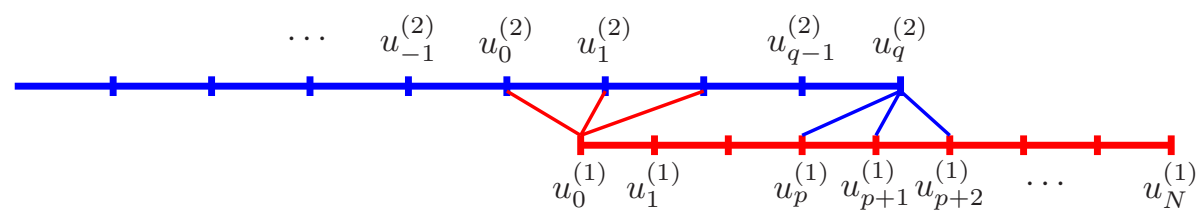

Figure 3: One-dimensional overlapping grid for the semi-infinite problem.

For purposes of the present analysis, we consider approximations of the wave equation that are continuous in time and discrete in space. A centered approximation is

$$
\begin{array}{ll}
\frac{\partial^{2} u_{j}^{(1)}}{\partial t^{2}}=\frac{u_{j+1}^{(1)}-2 u_{j}^{(1)}+u_{j-1}^{(1)}}{h_{1}^{2}}, \quad j=1,2, \ldots, N-1, \\
\frac{\partial^{2} u_{j}^{(2)}}{\partial t^{2}}=\frac{u_{j+1}^{(2)}-2 u_{j}^{(2)}+u_{j-1}^{(2)}}{h_{2}^{2}}, \quad j=\ldots, q-2, q-1,
\end{array}
$$

with initial conditions and boundary conditions

$$
\begin{aligned}
& u_{j}^{(1)}(0)=u_{0}\left(x_{j}^{(1)}\right), \quad \frac{\partial u_{j}^{(1)}}{\partial t}(0)=v_{0}\left(x_{j}^{(1)}\right), \quad j=0,1, \ldots, N, \\
& u_{j}^{(2)}(0)=u_{0}\left(x_{j}^{(2)}\right), \quad \frac{\partial u_{j}^{(2)}}{\partial t}(0)=v_{0}\left(x_{j}^{(2)}\right), \quad j=\ldots, q-1, q, \\
& u_{N}^{(1)}(t)=g(t), \quad\left|u_{j}^{(2)}(t)\right|<\infty,
\end{aligned}
$$

and interpolation conditions

$$
u_{0}^{(1)}=\sum_{k=0}^{r} a_{k} u_{k}^{(2)}, \quad u_{q}^{(2)}=\sum_{k=0}^{r} b_{k} u_{p+k}^{(1)} .
$$

Here $r+1$ is the number of grid points in the interpolation stencil, and the interpolation coefficients $a_{k}$ and $b_{k}$ are given by Lagrange interpolation. For example, $a_{0}=(1-\alpha)$ and $a_{1}=\alpha$ for linear interpolation $(r=1)$, and $a_{0}=\frac{1}{2}(1-\alpha)(2-\alpha), a_{1}=\alpha(2-\alpha)$ and $a_{2}=\frac{1}{2} \alpha(\alpha-1)$, for quadratic interpolation $(r=2)$, where $\alpha=\left(x_{0}^{(1)}-x_{0}^{(2)}\right) / h_{2}$ for both cases. The interpolation is restricted to be centered so that $\alpha \in\left[\frac{1}{2}(r-1), \frac{1}{2}(r+1)\right]$. The formulae for $b_{k}$ are defined in a similar fashion in terms of $\beta=\left(x_{q}^{(2)}-x_{p}^{(1)}\right) / h_{1}$.

Definition 1. We will say that the scheme (34)-(39) is stable if its solution remains uniformly bounded in time.

We note that more generally one can allow stable solutions that have bounded growth in time for any fixed time interval, but for the wave equation this more restrictive definition is used.

To analyze the stability of the scheme in (34)-(39), we apply a Laplace transform in time with dual variable $s$. From the general normal-mode theory [33] we are led to analyze the following eigenvalue problem:

$$
\begin{aligned}
& \left(s h_{1}\right)^{2} \tilde{u}_{j}^{(1)}=\tilde{u}_{j+1}^{(1)}-2 \tilde{u}_{j}^{(1)}+\tilde{u}_{j-1}^{(1)}, \quad j=1,2, \ldots, N-1, \\
& \left(s h_{2}\right)^{2} \tilde{u}_{j}^{(2)}=\tilde{u}_{j+1}^{(2)}-2 \tilde{u}_{j}^{(2)}+\tilde{u}_{j-1}^{(2)}, \quad j=\ldots, q-2, q-1, \\
& \tilde{u}_{N}^{(1)}=0, \quad\left|\tilde{u}_{j}^{(2)}\right|<\infty, \\
& \tilde{u}_{0}^{(1)}=\sum_{k=0}^{r} a_{k} \tilde{u}_{k}^{(2)}, \quad \tilde{u}_{q}^{(2)}=\sum_{k=0}^{r} b_{k} \tilde{u}_{p+k}^{(1)} .
\end{aligned}
$$

Here $s$ is the complex-valued eigenvalue while $\tilde{u}_{j}^{(1)}$ and $\tilde{u}_{j}^{(2)}$ are components of the discrete eigenfunction. A necessary condition for stability, known as the Godunov-Ryabenkii condition, is that there are no solutions to the eigenvalue problem in (40)-(43) with $\operatorname{Re}(s)>0$ as stated in the following theorem.

Theorem 1. The scheme (34)-(39) is unstable if there exists a nontrivial solution to the eigenvalue problem (40)-(43) with $\operatorname{Re}(s)>0$. 
Proof. If there is a solution to the eigenvalue problem (40)-(43), then there will be a homogeneous solution to (34)-(39) which grows like $e^{s t}$. If $\operatorname{Re}(s)>0$, then this solution will not be bounded in time $\square$.

The next lemma follows from the fact that the eigenvalue problem only depends on the product $s h_{m}$.

Lemma 1. If there is a solution to the eigenvalue problem (40)-(43) for given grid parameters $\left(s, h_{1}, h_{2}, r, \alpha, \beta, N\right)$, then for any $\gamma>0$ there is another solution (on a different grid) with parameters $\left(s \gamma, h_{1} / \gamma, h_{2} / \gamma, r, \alpha, \beta, N\right)$.

The growth rate on the new grid with mesh spacings $\left(h_{1} / \gamma, h_{2} / \gamma\right)$ would be $e^{\gamma \operatorname{Re}(s) t}$. Therefore, if there exists a solution to the eigenvalue problem with $\operatorname{Re}(s)>0$, then solutions exist on finer grids that grow more rapidly. This result indicates that if there are unstable modes for one mesh size, then as the mesh is refined there would be unstable modes with growth rates proportional to $1 / h_{m}$, i.e. $\left|u_{j}^{(m)}\right| \sim e^{\left(\gamma / h_{m}\right) t}$ with $\gamma>0$.

We now consider solutions of the eigenvalue problem in (40)-(43) to determine whether the scheme in (34)-(39) is unstable. We begin with the equations in (40) and (41), and examine fundamental solutions of the form $\tilde{u}_{j}^{(m)}=\widetilde{\kappa}_{m}^{j}, m=1,2$, where $\widetilde{\kappa}_{m}$ is a complex number that depends on $s h_{m}$. It follows that $\widetilde{\kappa}_{m}$ satisfies

$$
\widetilde{\kappa}_{m}^{2}-\left(2+z_{m}\right) \widetilde{\kappa}_{m}+1=0, \quad z_{m}=\left(s h_{m}\right)^{2},
$$

which has roots $\widetilde{\kappa}_{m}=\kappa_{m}^{ \pm}$, where

$$
\kappa_{m}^{ \pm}=1+z_{m} / 2 \pm \sqrt{z_{m}+z_{m}^{2} / 4}, \quad-\pi / 2 \leq \arg \left(\sqrt{z_{m}+z_{m}^{2} / 4}\right)<\pi / 2 .
$$

Let $s h_{m}=\xi+i \eta$, and define

$$
\kappa_{m}= \begin{cases}\kappa_{m}^{-}, & \text {for } \eta^{2} \leq \xi^{2}+2, \\ \kappa_{m}^{+}, & \text {for } \eta^{2}>\xi^{2}+2,\end{cases}
$$

so that $\left|\kappa_{m}\right| \leq 1$ for $\operatorname{Re}(s)>0$. The modulus of $\kappa_{m}\left(s h_{m}\right)$ is plotted in Figure 4 . We note that $\left|\kappa_{m}\left(s h_{m}\right)\right|=1$ for $s h_{m}=0^{+}+i \eta$ with $-2 \leq \eta \leq 2$. Also, we note that $\kappa_{m}\left(s h_{m}\right) \sim 1 / z_{m}$ as $z_{m} \rightarrow \infty$ with $\operatorname{Re}(s)>0$ (which can be seen from an asymptotic analysis of (44)).
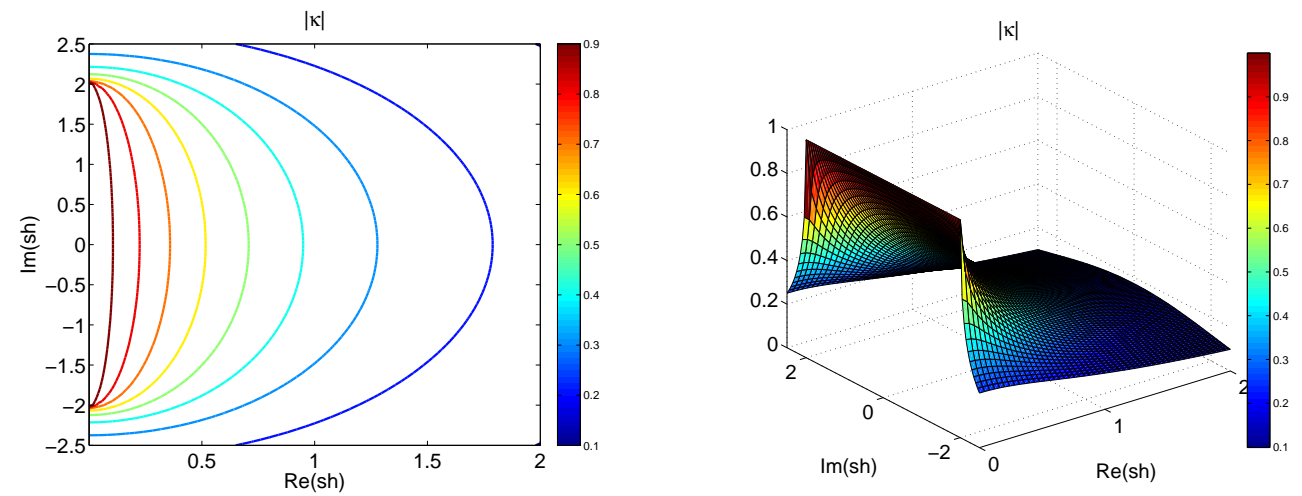

Figure 4: Contours and surface plot of $|\kappa|$ from the second-order wave equation for the root $\kappa(s h)$ with $|\kappa| \leq 1$ for $\operatorname{Re}(s)>0$. The modulus of $\kappa$ is one along the imaginary axis for $\operatorname{Im}(s h) \in[-2,2]$ and decays to zero as $\operatorname{Re}(s h) \rightarrow \infty$.

General solutions of equations (40) and (41) satisfying the boundary conditions have the form

$$
\begin{array}{ll}
\tilde{u}_{j}^{(1)}=A\left(\kappa_{1}^{j}-\kappa_{1}^{2 N-j}\right), & j=0,1,2, \ldots, N, \\
\tilde{u}_{j}^{(2)}=B \kappa_{2}^{q-j}, & j=\ldots, q-1, q,
\end{array}
$$

where $\kappa_{m}$ is defined in (45), and where $A=A(s)$ and $B=B(s)$ are bounded and spatially constant. Imposition of the interpolation equations implies

$$
\begin{gathered}
A\left(1-\kappa_{1}^{2 N}\right)=B \sum_{k=0}^{r} a_{k} \kappa_{2}^{q-k}, \\
B=A \sum_{k=0}^{r} b_{k}\left(\kappa_{1}^{p+k}-\kappa_{1}^{2 N-(p+k)}\right) .
\end{gathered}
$$


The condition for nontrivial solutions of (48), and therefore nontrivial solutions of the eigenvalue problem in (40)-(43), is that there be zeros of the following determinant for $\operatorname{Re}(s)>0$ :

$$
\begin{aligned}
G_{2}(s) & =\operatorname{det}\left[\begin{array}{cc}
\kappa_{1}^{2 N}-1 & \sum_{k=0}^{r} a_{k} \kappa_{2}^{q-k} \\
\sum_{k=0}^{r} b_{k}\left(\kappa_{1}^{p+k}-\kappa_{1}^{2 N-(p+k)}\right) & -1
\end{array}\right], \\
& =1-\kappa_{1}^{2 N}-\left(\sum_{k=0}^{r} a_{k} \kappa_{2}^{r-k}\right)\left(\sum_{k=0}^{r} b_{k}\left(\kappa_{1}^{k}-\kappa_{1}^{2 N-2 p-k}\right)\right) \kappa_{2}^{q-r} \kappa_{1}^{p} .
\end{aligned}
$$

In the next subsection below, we show that there are, in general, solutions to $G_{2}(s)=0$ with $\operatorname{Re}(s)>0$. We first prove the following lemma:

Lemma 2. The growth rate of solutions to the eigenvalue problem with $G_{2}(s)=0$ are bounded by $e^{(\gamma / h) t}$, where $\gamma=\gamma(r) \geq 0$ is a bounded constant that only depends on $r$.

Proof. Note that $\left|\kappa_{m}\left(s h_{m}\right)\right| \rightarrow 0$ as $\operatorname{Re}(s) \rightarrow \infty$. Thus for any $\epsilon>0$, we can find $\gamma>0$ such that $\left|\kappa_{m}\right|<\epsilon$ for $\operatorname{Re}\left(s h_{m}\right)>\gamma$. Here $\gamma$ does not depend on $h_{m}$. For example, from Figure 4 we see that $\left|\kappa_{m}\left(s h_{m}\right)\right|<\frac{1}{2}$ for $\operatorname{Re}\left(s h_{m}\right)>1$. Assuming $2 N-2 p-r \geq 0$ and $p+q>r$, and using $\left|\kappa_{m}\right|<\epsilon \leq 1$ it follows that

$$
\begin{aligned}
\left|\left(\sum_{k=0}^{r} a_{k} \kappa_{2}^{r-k}\right)\left(\sum_{k=0}^{r} b_{k}\left(\kappa_{1}^{k}-\kappa_{1}^{2 N-2 p-k}\right)\right) \kappa_{2}^{q-r} \kappa_{1}^{p}\right| & \leq\left(\sum_{k=0}^{r}\left|a_{k}\right|\right) 2\left(\sum_{k=0}^{r}\left|b_{k}\right|\right)\left|\kappa_{2}\right|^{q-r}\left|\kappa_{1}\right|^{p}, \\
& \leq 2 C_{r}^{2} \epsilon^{p+q-r},
\end{aligned}
$$

where

$$
C_{r}=\max _{-\frac{1}{2} \leq \alpha-r / 2 \leq \frac{1}{2}} \sum_{k=0}^{r}\left|a_{k}(\alpha)\right| .
$$

For example, $C_{1}=1$ for linear interpolation while $C_{2}=5 / 4$ for quadratic interpolation. We can thus choose $\epsilon$ small enough so that $\epsilon^{2 N}<\frac{1}{2}$ and $2 C_{r}^{2} \epsilon^{p+q-r}<\frac{1}{2}$ and thus $\left|G_{2}(s)\right|>0$. This proves the lemma $\square$.

For the case of an infinite interval, $b \rightarrow \infty$ (i.e. letting $N \rightarrow \infty$ in the above formula), and linear interpolation it can be shown that there are no solutions with $\operatorname{Re}(s)>0$, but that there are solutions with $\operatorname{Re}(s)=0$ and $\left|\kappa_{m}\right|=1$. These latter solutions are called generalized eigenvalues. As a result, there are no exponentially growing solutions for the infinite-interval case with linear interpolation, although there could be unstable modes that grow more slowly (corresponding to the generalized eigenvalues). This result for the second-order wave equation corresponds to a similar result for the first-order wave equation with linear interpolation on an infinite interval [23]. For quadratic interpolation on the infinite interval, there are also generalized eigenvalues with $\operatorname{Re}(s)=0$, but we do not know whether there exists any roots with $\operatorname{Re}(s)>0$. On the semi-infinite interval, there are roots with $\operatorname{Re}(s)>0$ (and thus unstable modes) as demonstrated in the next section.

\subsubsection{An unstable solution for the second-order wave equation}

For a given overlapping grid with parameters $\left(h_{1}, h_{2}, r, \alpha, \beta, p, q, N\right)$ there may or may not be a solution to $G_{2}(s)=0$ with $\operatorname{Re}(s)>0$. A search of the parameter space can be used to locate solutions. In this subsection, we provide an example of a solution with a relatively large value for $\operatorname{Re}(s)$ that arises on an overlapping grid with parameters that are typical of grids we use in practice. (This solution being one of many solutions we have found in our numerical search of the parameter space.) For the grid parameters

$$
h_{1}=1, \quad h_{2} \approx 1.4445, \quad r=2, \quad \alpha \approx 1.4408, \quad \beta \approx 1.2527, \quad p=1, \quad q=3, \quad N=7,
$$

we solve $G_{2}(s)=0$ numerically to find the complex root $s \approx(0.01747,1.251)$. For this case, the right boundary is located at $b=h_{1} N=7$ and the width of the overlap is $x_{q}^{(2)}-x_{0}^{(1)} \approx 2.2524$. The real and imaginary parts of the eigenfunction, $\bar{u}_{j}^{(m)}, m=1,2$, corresponding to this root are shown in the plot on the left in Figure 5. We note that while the real parts of $\bar{u}_{j}^{(1)}$ and $\bar{u}_{j}^{(2)}$ match reasonably well in the overlap region, the imaginary parts do not. In fact, the concavity of the imaginary parts of the two grid functions appear to have opposite signs in the overlap region. In our investigation of solutions of $G_{2}(s)=0$, this mismatch seems to be typical of unstable modes. 
We can also compute all of the eigenvalues of the matrix for an associated problem on a finite domain. For this problem, the domain is truncated on the left and the boundary condition $\tilde{u}_{q-M}^{(2)}=0$ is specified. Here, the positive integer $M$ determines position of the left boundary of the truncated domain. Using the same grid parameters in (50), we find that the finite-domain problem has a complex eigenvalue that converges to the eigenvalue belonging to the semi-infinite domain problem as $M$ gets large. For example, the eigenvalues of the finite-domain problem for $M=112$ are shown in the plot on the right in Figure 5 . Here, we note that there is an unstable complex eigenvalue which agrees with the infinite-domain value given above to within a relative error of $0.01 \%$.
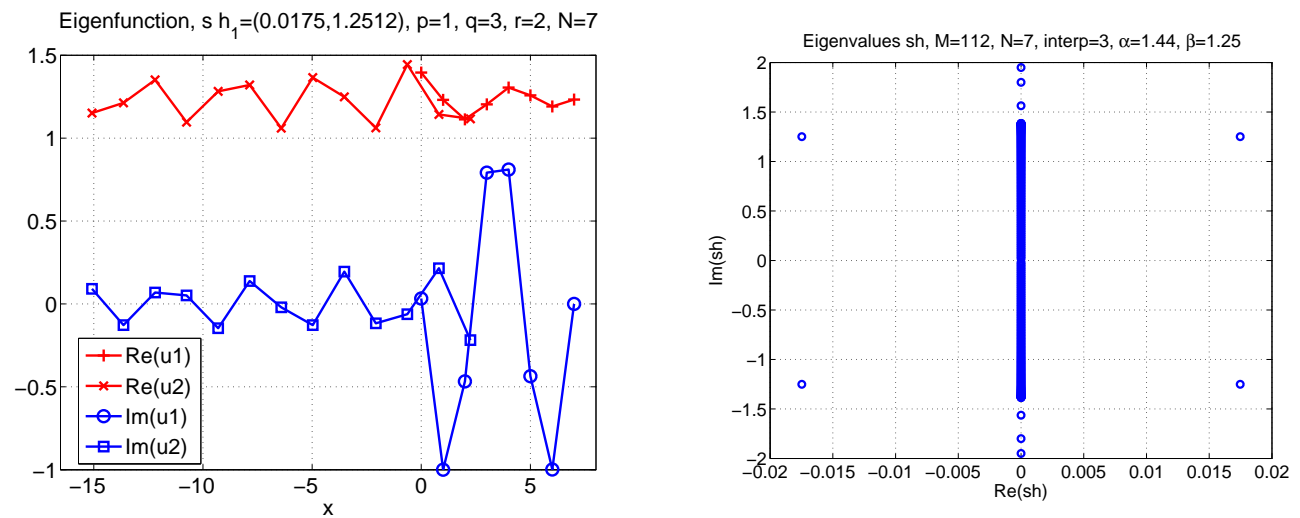

Figure 5: Second-order wave equation. Left: Overlapping grid eigenfunction for the unstable eigenvalue $s \approx(0.01747,1.251)$, $N=7$ on the semi-infinite interval. The real part of the eigenfunction has been shifted upward for clarity. Right: The eigenvalues $s$ of the finite domain overlapping grid problem with $N=7$ and $M=112$. The finite domain problem has a complex eigenvalue $s \approx(0.01747,1.251)$ which agrees with that of the semi-infinite domain value.

\subsubsection{Artificial dissipation and high-order filtering}

We can stabilize the scheme (34)-(39) for the scalar wave equation by adding artificial dissipation. The dissipation term we employ is analogous to that for the second-order system for linear elasticity, and when added to the right-hand side of the wave equation gives a modified equation of the form

$$
\frac{\partial^{2} u}{\partial t^{2}}=\frac{\partial^{2} u}{\partial x^{2}}-\frac{a_{d}}{h}\left(-h^{2} \frac{\partial^{2}}{\partial x^{2}}\right)^{d / 2} \frac{\partial u}{\partial t}
$$

where $d$ is a positive even integer defining the order of the dissipation and $a_{d} / h$ is a dissipation coefficient. A fully discrete approximation is

$$
\frac{u_{j}^{n+1}-2 u_{j}^{n}+u_{j}^{n-1}}{\Delta t^{2}}=\frac{u_{j+1}^{n}-2 u_{j}^{n}+u_{j-1}^{n}}{h^{2}}-\frac{a_{d}}{h}\left(-\Delta_{+} \Delta_{-}\right)^{d / 2} \frac{u_{j}^{n}-u_{j}^{n-1}}{\Delta t},
$$

or

$$
u_{j}^{n+1}=2 u_{j}^{n}-u_{j}^{n-1}+\sigma^{2}\left(u_{j+1}^{n}-2 u_{j}^{n}+u_{j-1}^{n}\right)-\sigma a_{d}\left(-\Delta_{+} \Delta_{-}\right)^{d / 2}\left(u_{j}^{n}-u_{j}^{n-1}\right),
$$

where $\sigma=\Delta t / h$. We note that the discrete scheme depends on $\Delta t$ and $h$ only through $\sigma$ and thus the dissipation only depends on $\sigma$ as the mesh is refined. The dissipation coefficient increases like $1 / h$ as the mesh is refined, assuming that $a_{d}$ is a constant independent of the mesh spacing. As mentioned previously in the discussion of the SOS scheme (see Section 4.1), the factor of $1 / h$ is not needed for weaker instabilities where the growth rate remains bounded as $h$ goes to zero. Here, the instability is stronger so that the factor is needed.

The symbol or amplification factor, $\mathcal{A}_{d}$, for the scheme in $(52)$ on a periodic domain is given by the quadratic equation

$$
\mathcal{A}_{d}^{2}=\left(2-4 \sigma^{2} \sin ^{2}(\xi / 2)-\sigma a_{d}\left(4 \sin ^{2}(\xi / 2)\right)^{d / 2}\right) \mathcal{A}_{d}-\left(1-\sigma a_{d}\left(4 \sin ^{2}(\xi / 2)\right)^{d / 2}\right),
$$

where $|\xi| \leq \pi$. The plot on the left in Figure 6 shows $\left|\mathcal{A}_{d}\right|$ for the case of fourth-order dissipation, i.e. $d=4$, with $a_{d}=1 / 8$ for various values of $\sigma$. The magnitude of the amplification factor determines the damping (per time step) of each Fourier mode. With no dissipation, $a_{d}=0$, and with $\sigma \leq 1$, there is no damping, $\left|\mathcal{A}_{d}\right|=1$, for all $\xi$. For $a_{d}>0$ the scheme is stable and there is damping of the high-frequency Fourier modes 
provided $\sigma \leq \sigma_{0}$, where the stability bound $\sigma_{0}$ depends on $d$ and $a_{d}$. For example, we find that $\sigma_{0} \approx 0.617$ for $d=4$ and $a_{d}=1 / 8$. The plot of $\left|\mathcal{A}_{d}\right|$ shows that the highest frequency mode on the mesh (i.e. the plus-minus mode with $|\xi|=\pi$ ) is damped completely when $\sigma=0.5$. For $\sigma=0.6$ there is less damping of the high-frequency modes, and for $\sigma>\sigma_{0}$ the scheme is unstable.
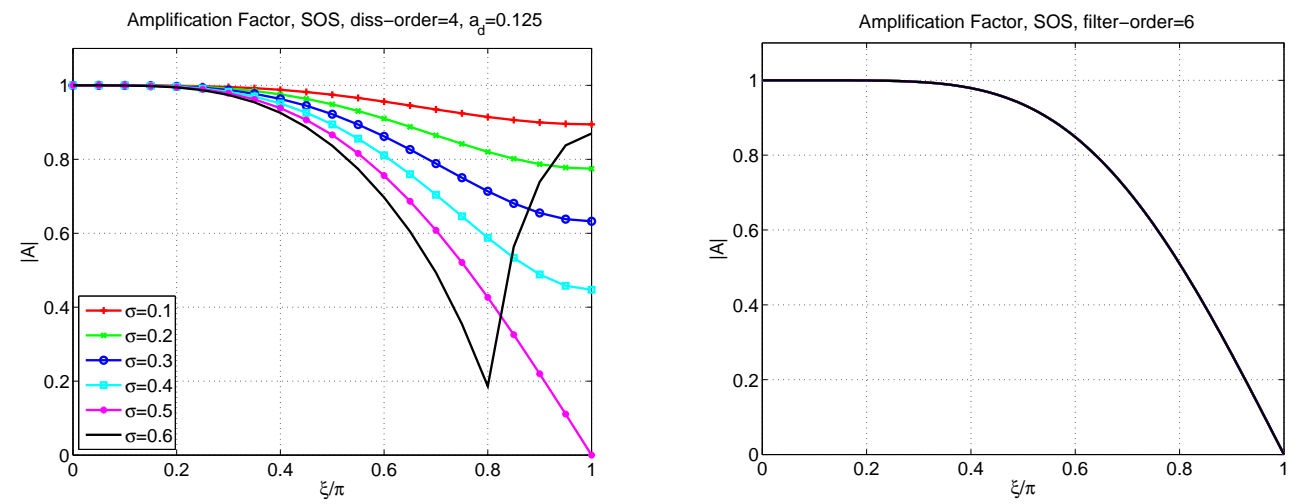

Figure 6: The magnitude of the amplification factor as a function of $\sigma=\Delta t / h$ and the normalized wave-number, $\xi / \pi$, for the SOS scheme for the one-dimensional second-order wave equation on a periodic domain. Left: SOS scheme with a fourth-order artificial dissipation. Right: SOS scheme with a sixth-order filter. The magnitude of the amplification factor for the filter scheme is independent of $\sigma$.

We now reconsider the behaviour of the unstable mode given in Section 5.1.1 when dissipation is applied. For the grid parameters in (50), we determine the eigenvalues to the finite-domain overlapping-grid problem numerically with second or fourth-order dissipation added. The results, shown in Figure 7, indicate that the scheme can be stablized with second-order dissipation $\left(d=2, a_{d}=.025\right)$ or fourth-order dissipation $(d=4$, $\left.a_{d}=.05\right)$. As expected, the addition of dissipation acts to shift the unstable eigenvalues to the left-half plane, and the eigenvalues on the imaginary axis representing high-frequency neutrally-stable modes bend over to the left-half plane to acquire some damping. Note that in the case of the fourth-order dissipation, the wide stencil requires special treatment at the interior points next to an interpolation or boundary point. At these four interior points (two points on each grid) we simply turn off the dissipation. Alternatively, one-sided approximations for the dissipation could be used at these points.
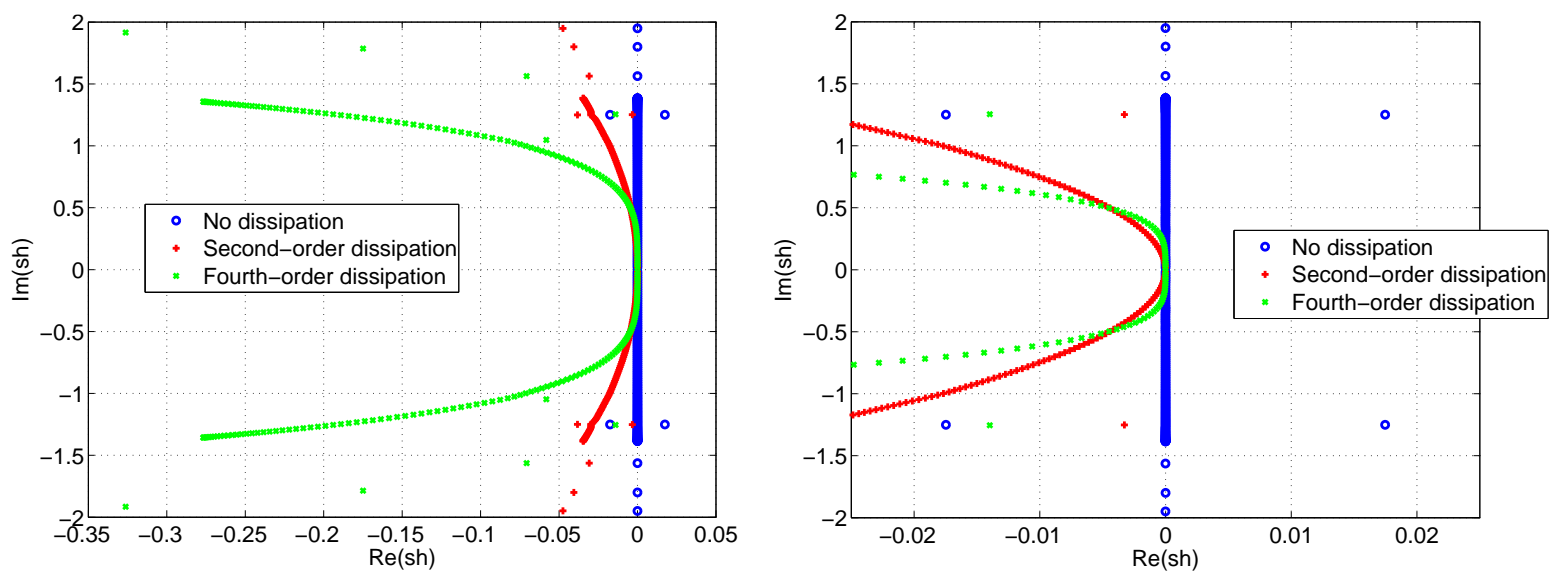

Figure 7: Second-order wave equation on a one-dimensional overlapping grid. Eigenvalues of the finite-domain problem computed with no-dissipation, with second-order dissiaption $\left(a_{d}=.025\right)$ and fourth-order dissipation $\left(a_{d}=.05\right)$. Right: enlarged view of the figure on the left. The scheme is stabilized with the addition of dissipation as there are no eigenvalues with positive real part.

The high-order filter, discussed in Section 4.1, provides another approach to add dissipation and stabilize the scheme. The fully discrete scheme with the high-order filter is a two step process consisting of a centered scheme for the wave equation given by

$$
u_{j}^{*}=2 u_{j}^{n}-u_{j}^{n-1}+\sigma^{2}\left(u_{j+1}^{n}-2 u_{j}^{n}+u_{j-1}^{n}\right),
$$

and a filter given by

$$
u_{j}^{n+1}=u_{j}^{*}-\beta_{f}\left(-\Delta_{+} \Delta_{-}\right)^{d / 2} u_{j}^{*},
$$


where $d$ is the order of the filter and $\beta_{f}$ is a coefficient. (An analogous form for the SOS scheme is given in (21).) The amplification factor, $\mathcal{A}_{f}$, for the scheme in (54) and (55) on a periodic domain is determined by

$$
\mathcal{A}_{f}^{2}=\mathcal{T}_{f}\left[\left(2-4 \sigma^{2} \sin ^{2}(\xi / 2)\right) \mathcal{A}_{f}-1\right],
$$

where $\mathcal{T}_{f}=1-\beta_{f}\left(4 \sin ^{2}(\xi / 2)\right)^{d / 2}$ is the transfer function for the filter. If $\Delta t$ is chosen so that the unfiltered scheme is stable, i.e. if $|\sigma| \leq 1$, then the filtered scheme satisfies $\left|\mathcal{A}_{f}\right|=\left|\mathcal{I}_{f}\right|$. Thus, the filtered scheme given by (54) and (55) is stable under the same time-step restriction as the unfiltered scheme provided $\left|\mathcal{T}_{f}\right| \leq 1$. The filtered scheme, therefore, has an advantage over the artificial-dissipation scheme since the former does not require a reduced time step and its damping characteristics do not depend on $\sigma$. The plot on the right in Figure 6 shows the magnitude of the $\mathcal{A}_{f}$ for the sixth-order filter, $d=6$, with the choice $\beta_{f}=1 / 2^{d}$ (i.e. the choice that completely damps the highest frequency Fourier mode).

While the analysis presented here does not rigorously apply to the full overlapping grid problem, it does give an indication of how the addition of dissipation or the application of a filter can act to stabilize the scheme. We remark that in all our simulations for the full elastodynamic equations, including those presented in Section 6, we have not found any case where the scheme could not be stabilized. In general we recommend using a fourth-order dissipation (19) with coefficient $\alpha_{4}=1$, together with a sixth-order filter (21) with $\beta_{6}=1 / 128$. We emphasize that the formal order of accuracy of the scheme remains second-order, even with the addition of this dissipation and filter.

\subsection{Stability of the first-order wave equation}

We now consider the first-order scalar wave equation for $u(x, t)$ defined on $\Omega=(-\infty, b]$ for $t \geq 0$, namely,

$$
\begin{aligned}
\frac{\partial u}{\partial t} & =\frac{\partial u}{\partial x}, & & x \in(-\infty, b), t>0, \\
u(x, 0) & =u_{0}(x), & & x \in(-\infty, b), \\
u(b, t) & =g(t), & & t>0 .
\end{aligned}
$$

As before, we consider an approximation that is continuous in time and uses centered differences in space. For the overlapping grid shown in Figure 3, the approximation becomes

$$
\begin{aligned}
\frac{\partial u_{j}^{(1)}}{\partial t} & =\frac{u_{j+1}^{(1)}-u_{j-1}^{(1)}}{2 h_{1}}, \quad j=1,2, \ldots, N-1, \\
\frac{\partial u_{j}^{(2)}}{\partial t} & =\frac{u_{j+1}^{(2)}-u_{j-1}^{(2)}}{2 h_{2}}, \quad j=\ldots, q-2, q-1, \\
u_{j}^{(1)}(0) & =u_{0}\left(x_{j}^{(1)}\right), \quad j=0,1, \ldots, N, \\
u_{j}^{(2)}(0) & =u_{0}\left(x_{j}^{(2)}\right), \quad j=\ldots, q-1, q, \\
u_{N}^{(1)}(t) & =g(t),
\end{aligned}
$$

with the interpolation formulas

$$
u_{0}^{(1)}=\sum_{k=0}^{r} a_{k} u_{k}^{(2)}, \quad u_{q}^{(2)}=\sum_{k=0}^{r} b_{k} u_{p+k}^{(1)} .
$$

In order to study the stability of the approximation, we employ a Laplace transform to obtain the eigenvalue problem

$$
\begin{aligned}
& 2\left(s h_{1}\right) \tilde{u}_{j}^{(1)}=\tilde{u}_{j+1}^{(1)}-\tilde{u}_{j-1}^{(1)}, \quad j=1,2,3, \ldots, N-1, \\
& 2\left(s h_{2}\right) \tilde{u}_{j}^{(2)}=\tilde{u}_{j+1}^{(2)}-\tilde{u}_{j-1}^{(2)}, \quad j=q-1, q-2, \ldots, \\
& \tilde{u}_{N}^{(1)}=0, \quad\left|\tilde{u}_{j}^{(2)}\right|<\infty, \\
& \tilde{u}_{0}^{(1)}=\sum_{k=0}^{r} a_{k} \tilde{u}_{k}^{(2)}, \quad \tilde{u}_{q}^{(2)}=\sum_{k=0}^{r} b_{k} \tilde{u}_{p+k}^{(1)} .
\end{aligned}
$$


Solutions of the difference equations in (62) and (63), and satisfying the conditions in (64), are

$$
\begin{array}{ll}
\tilde{u}_{j}^{(1)}=A\left(\kappa_{1}^{j}-(-1)^{N}\left(-\kappa_{1}\right)^{2 N-j}\right), & j=0,1, \ldots, N \\
\tilde{u}_{j}^{(2)}=B\left(-\kappa_{2}\right)^{q-j}, & j=\ldots, q-1, q,
\end{array}
$$

where $\kappa_{m}=\kappa_{m}\left(s h_{m}\right), m=1,2$, are roots of the characteristic equation $\kappa^{2}-2\left(s h_{m}\right) \kappa-1=0$ with $\left|\kappa_{m}\left(s h_{m}\right)\right| \leq 1$ for $\operatorname{Re}(s)>0$, and where $A=A(s)$ and $B=B(s)$ are bounded and spatially constant. The modulus of $\kappa_{m}\left(s h_{m}\right)$ is similar in behavior to that shown earlier in Figure 4 for the case of the second-order wave equation.

Following the steps used previously for the second-order wave equation, we use the solutions in (66) and (67) and the interpolation formulas in (61) to obtain the determinant condition $G_{1}(s)=0$, where

$$
G_{1}(s)=1-(-1)^{N} \kappa_{1}^{2 N}-\left(\sum_{k=0}^{r} a_{k}\left(-\kappa_{2}\right)^{q-k}\right)\left(\sum_{k=0}^{r} b_{k}\left(\kappa_{1}^{p+k}-(-1)^{N}\left(-\kappa_{1}\right)^{2 N-p-k}\right)\right) .
$$

A necessary condition for stability of solutions to (56)-(61) is that there are no roots of $G_{1}(s)$ with $\operatorname{Re}(s)>0$. The next two results for the first-order wave equation are analogous to those in Lemmas 1 and 2 for the second-order wave equation.

Lemma 3. If there is a solution to the eigenvalue problem (62)-(65) for given grid parameters $\left(s, h_{1}, h_{2}, r, \alpha, \beta, N\right)$, then for any $\gamma>0$ there is another solution (on a different grid) with parameters $\left(s \gamma, h_{1} / \gamma, h_{2} / \gamma, r, \alpha, \beta, N\right)$.

Proof. The proof follows that of Lemma $1 \square$.

Lemma 4. The growth rate of solutions to the eigenvalue problem with $G_{1}(s)=0$ are bounded by $e^{(\gamma / h) t}$ where $\gamma=\gamma(r) \geq 0$ is a bounded constant that only depends on $r$.

Proof. The proof follows that of Lemma 2 since $\kappa_{m} \rightarrow 0$ as $\operatorname{Re}(s) \rightarrow \infty \square$.

We note that Olsson and Petersson [23] performed a similar stability analysis for a first-order wave equation. For the infinite domain case with linear interpolation, they showed that there are no eigenvalues with $\operatorname{Re}(s)>0$, but that there are generalized eigenvalues with $\operatorname{Re}(s)=0$ for the special case when $\alpha=\beta=0$ (i.e. when the endpoint of component grid 1 overlaps exactly with a grid point on grid 2 , and vice versa, and when the spacing for one grid is an integer multiple of the other). These generalized eigenvalues occur for any order of interpolation since the interpolation is always exact when $\alpha=\beta=0$. For the finite-domain case, they found roots with $\operatorname{Re}(s)>0$. As the mesh was refined (with $N$ increasing so that the boundary of the finite domain remains fixed), the real part of some of these roots increased at a rate proportional to $1 / h^{\gamma}$, where $\gamma \approx .065$ or $\gamma \approx .033$ for the two cases presented in their paper. They also found roots whose real part decreased as the mesh was refined.

For the semi-infinite domain problem considered here, with linear or quadratic interpolation, we also find roots with $\operatorname{Re}(s)>0$ corresponding to unstable modes. The difference between the work here and that in [23] is that we consider the case where $N$ is held fixed as the mesh is refined. This corresponds to the situation where the grid overlap moves closer to the fixed boundary as the mesh is refined. For this case, we know analytically that the roots increase at a rate proportional to $1 / h$ as the mesh is refined, a rate higher than that found in [23] for the finite-domain case. Thus, the evidence suggests that the case of fixed $N$ is the more difficult from the point of view of stability as compared to the case when $N$ is increased as the mesh is refined.

\subsubsection{Unstable solutions for the first-order wave equation}

We present two examples of unstable modes for the first-order wave equation that are determined by the roots of $G_{1}(s)$ in (68). We first consider the case of linear interpolation. For the grid parameters

$$
h_{1}=1, \quad h_{2} \approx 1.5025, \quad r=1, \quad \alpha \approx 0.0033278, \quad \beta=0, \quad p=2, \quad q=3, \quad N=5,
$$

we solve $G_{1}(s)$ numerically to find the root $s \approx(0.01108,0.4786)$ corresponding to an unstable mode. For this case, the right boundary is located at $b=h_{1} N=5$ and the width of the overlap is $x_{q}^{(2)}-x_{0}^{(1)}=3$. For the second case, we use quadratic interpolation and the grid parameters

$$
h_{1}=1, \quad h_{2} \approx 1.3900, \quad r=2, \quad \alpha \approx 1.2500, \quad \beta \approx 0.82250, \quad p=3, \quad q=4, \quad N=7,
$$


and find the unstable root $s \approx(0.02022,0.6701)$. For this second case, $b=7$ and $x_{q}^{(2)}-x_{0}^{(1)}=3.8225$. As done previously for the second-order wave equation, the examples here are confirmed by computing all of the eigenvalues of the matrix that results from a corresponding finite-domain problem with a large value for $M$ (see Section 5.1.1). The real and imaginary parts of the eigenfunctions for the two cases are shown in Figure 8. As before, we observe that the grid functions representing the eigenfunctions do not match well in the overlap region, which appears to be typical for unstable solutions.
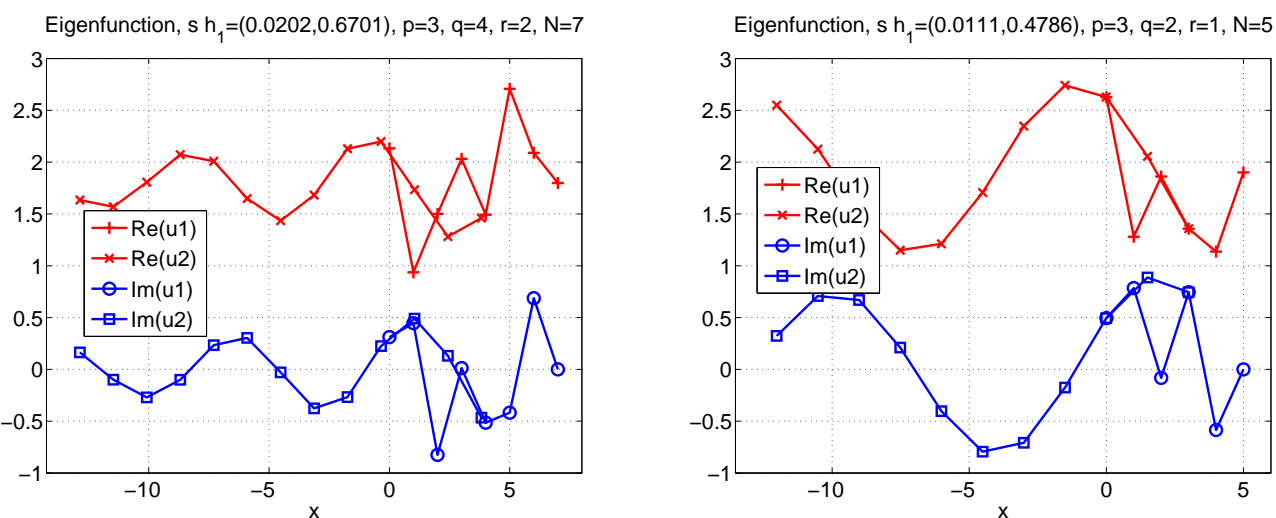

Figure 8: First-order wave equation. Left: Overlapping grid eigenfunction for the unstable mode $s \approx(0.02022,0.6701), N=7$ on the semi-infinite interval with quadratic interpolation. Right: Unstable mode for linear interpolation, $s \approx(0.01108,0.4786)$.

\subsubsection{Dissipation in the Godunov method}

The Godunov method described in Section 4.2 can be applied to the solution of the first-order scalar wave equation, $u_{t}=u_{x}$, to examine how the dissipation inherent in the scheme provides a means to stabilize the solution. The second-order unlimited scheme (using $\mathcal{L}(a, b)=(a+b) / 2$ ) is given by

$$
u_{j}^{n+1}=u_{j}^{n}+\sigma \Delta_{0} u_{j}^{n}+\frac{\sigma^{2}}{2} \Delta_{+} \Delta_{-} u_{j}^{n}-\frac{\sigma}{4}(1-\sigma) \Delta_{0} \Delta_{+} \Delta_{-} u_{j}^{n}-\frac{\sigma}{8}(1-\sigma)\left(\Delta_{+} \Delta_{-}\right)^{2} u_{j}^{n},
$$

where $\sigma=\Delta t / h$. The first three terms on the right-hand side of (69) comprise the Lax-Wendroff scheme, while the remaining terms provide upwinding and high-order dissipation. The amplification factor is given by

$$
\mathcal{A}_{g}=1-2 \sigma^{2} \sin ^{2}(\xi / 2)-2 \sigma(1-\sigma) \sin ^{4}(\xi / 2)+i \sigma \sin (\xi)\left(1+(1-\sigma) \sin ^{2}(\xi / 2)\right),
$$

where $|\xi| \leq \pi$. Whence,

$$
\left|\mathcal{A}_{g}\right|^{2}=1-2 \sigma(1-\sigma)\left(1+\sigma^{2}+(1-\sigma)^{2}\right) \sin ^{4}(\xi / 2)-4 \sigma^{2}(1-\sigma)^{2} \sin ^{6}(\xi / 2) .
$$

We note that $\left|\mathcal{A}_{g}\right|$ is unchanged if $\sigma$ is replaced by $1-\sigma$. For the well-known Lax-Wendroff scheme, by comparison, we have $\left|\mathcal{A}_{l w}\right|^{2}=1-4 \sigma^{2}\left(1-\sigma^{2}\right) \sin ^{4}(\xi / 2)$. Figure 9 shows the behavior of $\left|\mathcal{A}_{g}\right|$ and $\left|\mathcal{A}_{l w}\right|$ as a function of $\xi$ for values of $\sigma \in[1 / 2,1]$. The value $\sigma=1 / 2$ results in the most dissipation of high wave numbers for the Godunov scheme while the dissipation goes to zero when $\sigma=1$ and $\sigma=0$. Since the Godunov scheme damps the amplitudes of the high wave-number components in a similar fashion to the high-order filter (21) for the second-order scheme (see the discussion in Section 5.1.2), it might be expected that the inherent dissipation in the Godunov scheme is sufficient to stabilize the approach on an overlapping grid.

To illustrate the behaviour of the Godonov scheme on an overlapping grid we revisit the eigenvalue problem for the first-order wave equation considered in Section 5.2. We solve the eigenvalue problem given by equations (62), (63) and (65) except on a finite domain and with periodic boundary conditions. We consider the periodic problem since the results are clearer. For the overlapping grid case we use 25 points on each grid, quadratic interpolation and the grid parameters

$$
h_{1}=1, \quad h_{2}=1, \quad r=2, \quad \alpha=.5, \quad \beta=.5, \quad p=1, \quad q=2 .
$$



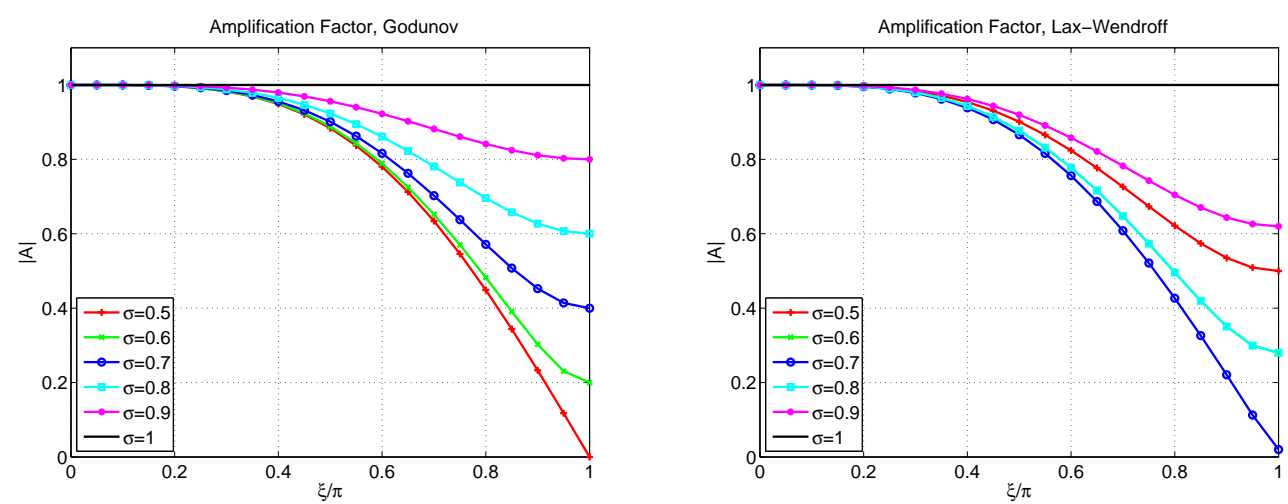

Figure 9: The magnitude of the amplification factor for the Godunov scheme, $\left|\mathcal{A}_{g}\right|$, and, for comparison, the Lax-Wendroff scheme, $\left|\mathcal{A}_{l w}\right|$, for the one-dimensional first-order wave equation on a periodic domain. Note that $\left|\mathcal{A}_{g}(\sigma)\right|=\left|\mathcal{A}_{g}(1-\sigma)\right|$ so that the curve for $\sigma=0.3$ for the Godunov scheme is the same as the curve for $\sigma=0.8$.

We also solve this problem with the time-continuous version of the Godunov scheme ${ }^{7}$, which after Laplace transforming becomes

$$
\begin{aligned}
\left(s h_{1}\right) \tilde{u}_{j}^{(1)} & =\Delta_{0} \tilde{u}_{j}^{(1)}-\frac{1}{4} \Delta_{0} \Delta_{+} \Delta_{-} \tilde{u}_{j}^{(1)}-\frac{1}{8}\left(\Delta_{+} \Delta_{-}\right)^{2} \tilde{u}_{j}^{(1)} \\
& =\left(\tilde{u}_{j+1}^{(1)}-\tilde{u}_{j-1}^{(1)}\right) / 2+\left(-\tilde{u}_{j+2}^{(1)}+3 \tilde{u}_{j+1}^{(1)}-3 \tilde{u}_{j}^{(1)}+\tilde{u}_{j-1}^{(1)}\right) / 4
\end{aligned}
$$

with a similar equation for $\tilde{u}_{j}^{(2)}$.

To better understand the overlapping grid results given below, we first consider the situation of a $2 \pi$ periodic domain discretized with a single grid of $N+1$ grid points, $x_{j}=j h, j=0,1, \ldots, N$, with grid spacing $h=2 \pi / N$. The discrete eigenvalues and eigenvectors for the $D_{0}$ operator on this grid satisfy $D_{0} w_{j}^{(m)}=s_{m} w_{j}^{(m)}$ and are given by

$$
s_{m} h=i \sin (2 \pi m / N), \quad w_{j}^{(m)}=\exp (2 \pi i m j / N), \quad \text { for } m=-\frac{N}{2},-\frac{N}{2}+1, \ldots, \frac{N}{2}-1,
$$

where for simplicity $N$ is taken as an even integer. Note that as $|m|$ increases from zero, $m=0, \pm 1, \pm 2, \ldots$, the eigenvalues first move away from the origin along the imaginary axis, reach a maximum modulus at $m= \pm N / 4$ and then decrease back to the origin. We call the eigenfunctions with $|m|>N / 4$ spurious modes since they are high-frequency modes in space but have eigenvalues with relatively small modulus. These spurious modes are purely numerical modes whose eigenvalues are unrelated to those of the continuous operator.

Figure 10 shows the computed eigenvalues for the centered and Godunov schemes for the overlapping grid problem. The centered scheme has a number of unstable modes for this grid configuration corresponding to the eigenvalues with positive real part. Most of the unstable modes for the centered scheme correspond to the spurious modes defined above. By comparison, the Godunov scheme has no unstable modes. The spurious modes now have significant damping since the corresponding eigenvalues have been shifted to have negative real parts. This example thus demonstrates that the dissipation inherent in the Godunov scheme can stabilize the overlapping grid problem. As a concluding remark for this section, we note that in practice, when solving the equations of elasticity in first-order form, we find that the Godunov scheme remains stable for all of the cases considered, including those described in Section 6 .

\section{Numerical results}

We now present numerical results to verify the correctness of the implementations of the SOS and FOS schemes, and to study and compare the accuracy, stability and performance of the two different approaches for a wide set of problems. These problems additionally provide valuable benchmark solutions for time dependent elasticity. In the first set of problems, we use the method of analytic solutions to construct exact solutions of the governing equations. This approach, as described in Appendix C, is used in Sections 6.1, 6.2

\footnotetext{
${ }^{7}$ The time continuous Godunov scheme is found by dividing equation (69) by $\Delta t$ and taking the limit $\Delta t \rightarrow 0$.
} 

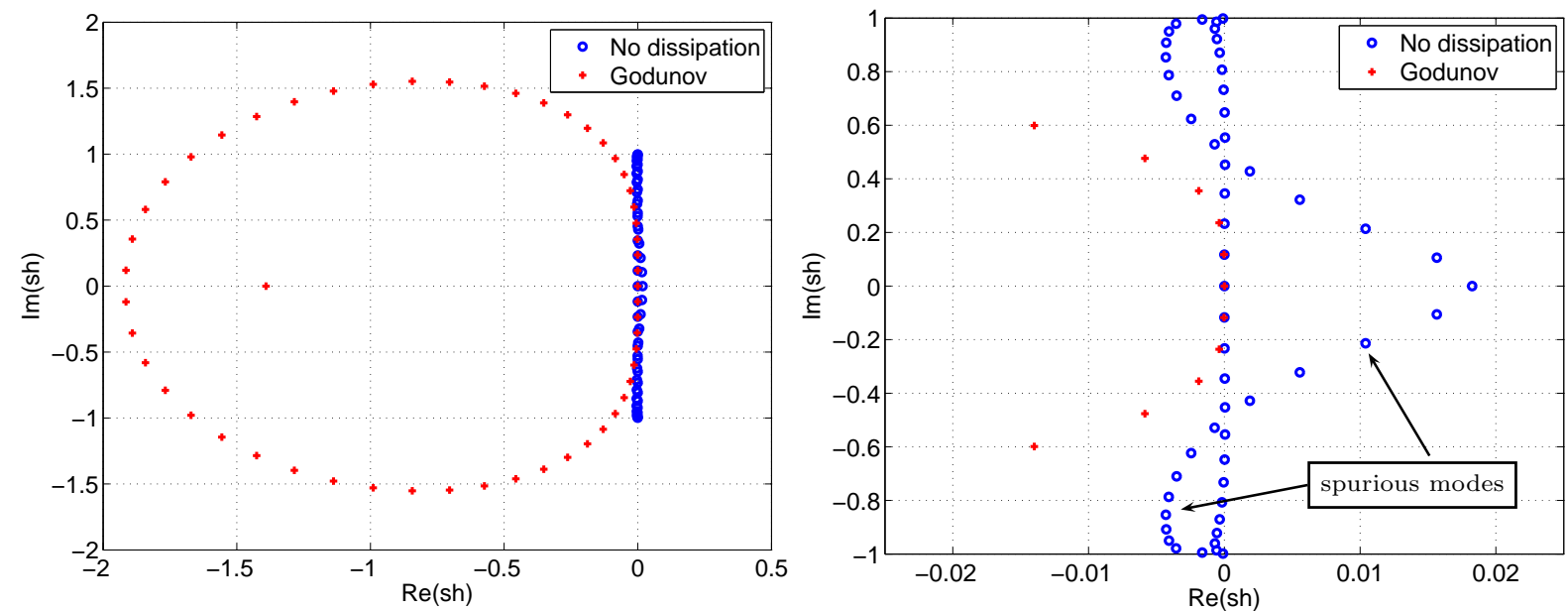

Figure 10: First-order wave equation on a one-dimensional overlapping grid. Eigenvalues of the periodic-domain problem computed with no-dissipation and with the Godunov upwind scheme. Right: enlarged view of the figure on the left. The upwind scheme is stable since there are no eigenvalues with positive real part.

and 6.3 for three different problems. The first of these problems involves an application in two dimensions on a circular disk. This problem is used, in part, to verify the stability results discussed in the previous section. The second of these problems involves calculations in a three-dimensional geometry consisting of a spherical cavity in a box, and the third problem considers a moving pulse solution in a two-dimensional domain. The second problem is used to check the parallel implementation of the schemes in three dimensions, while the third problem is used to check the AMR implementation of the code.

The problems discussed in Sections 6.4 and 6.5 involve two problems in which exact solutions of the governing equations are available (without applying fictitious forcing functions). These solutions involve vibrational modes in an annulus and a solid sphere. As with the previous problems, quantitative results that verify the accuracy of the two schemes are given.

The last two problems consider the diffraction of a p-wave "shock" by a circular cavity in two dimensions and the elastic response of a three-dimensional circular plate with holes. These two problems, discussed in Sections 6.6 and 6.7, respectively, illustrate the numerical schemes for complex configurations in which exact solutions are not known. The first of these problems shows the application of AMR on a composite grid for a non-smooth solution, and the second illustrates the numerical schemes for a problem in a complex three-dimensional geometry. A posteriori estimates of the error are computed for both cases to assess the accuracy of schemes.

A comparative study of the performance of the two schemes is discussed in Section 6.8. This is done using the calculation of a vibrational mode in a solid sphere as a representative problem. Finally, in Section 7 we discuss the accuracy of the schemes in terms of points per wavelengths.

\subsection{Stability and accuracy for an elastic disk}

The aim of this section is to illustrate the stability and accuracy of the SOS and FOS schemes for the equations of linear elasticity in two dimensions, and to relate the stability results for these equations to the results of the stability analysis performed in Section 5 for the first and second-order wave equations on a one-dimensional overlapping grid. For this illustration, we solve the equations of elasticity on a unit disk and apply the method of analytic solutions using trigonometric functions for the components of displacement, velocity and stress given by

$$
\begin{gathered}
\bar{u}_{1}=.5 c_{1} c_{2} c_{t}, \quad \bar{u}_{2}=.5 s_{1} c_{2} c_{t}, \quad \bar{v}_{1}=.75 s_{1} c_{2} c_{t}, \quad \bar{v}_{2}=.25 s_{1} s_{2} c_{t} \\
\bar{\sigma}_{11}=-.5 c_{1} c_{2} c_{t}, \quad \bar{\sigma}_{12}=.4 s_{1} c_{2} c_{t}, \quad \bar{\sigma}_{21}=.4 s_{1} c_{2} c_{t}, \quad \bar{\sigma}_{22}=.6 c_{1} s_{2} c_{t}
\end{gathered}
$$

where $\left(c_{j}, s_{j}\right)=\left(\cos \left(\pi x_{j}\right), \sin \left(\pi x_{j}\right)\right), j=1,2$, and $c_{t}=\cos (\pi t)$. We consider two composite grids (with varying grid resolution) for the unit disk $\Omega_{D}=\left\{\left(x_{1}, x_{2}\right) \mid x_{1}^{2}+x_{2}^{2} \leq 1\right\}$ as shown in Figure 11. Both composite grids use annular grids, which are defined by

$\mathcal{C}\left(\left[r_{a}, r_{b}\right], N_{1}, N_{2}\right)=\left\{\left(r_{i_{2}} \cos \left(\theta_{i_{1}}\right), r_{i_{2}} \sin \left(\theta_{i_{1}}\right)\right) \mid \theta_{i_{1}}=2 \pi i_{1} / N_{1}, r_{i_{2}}=r_{a}+\left(r_{b}-r_{a}\right) i_{2} / N_{2}, i_{k}=0,1, \ldots, N_{k}, k=1,2\right\}$. 
The interior of the domain is covered with a Cartesian grid, which is defined by

$$
\mathcal{R}\left(\left[x_{a}, x_{b}\right] \times\left[y_{a}, y_{b}\right], N_{1}, N_{2}\right)=\left\{\left(x_{a}+\left(x_{b}-x_{a}\right) i_{1} / N_{1}, y_{a}+\left(y_{b}-y_{a}\right) i_{2} / N_{2}\right) \mid i_{k}=0,1, \ldots, N_{k}, k=1,2\right\} .
$$

A composite grid for the disk is given by

$$
\mathcal{G}_{D}^{(j)}=\mathcal{R}\left(\left[R_{\text {in }}-h_{j}, R_{\text {in }}+h_{j}\right]^{2}, N_{x}(j), N_{x}(j)\right) \cup \mathcal{C}\left(\left[R_{\text {in }}, 1\right], N_{\theta}(j), N_{r}(j)\right),
$$

where the number of grid cells in the various coordinate directions are given by

$$
N_{x}(j)=\left\lfloor 2\left(R_{\mathrm{in}}+h_{j}\right) / h_{j}+1.5\right\rfloor, \quad N_{\theta}(j)=\left\lfloor\pi\left(1+R_{\mathrm{in}}\right) / h_{j}+1.5\right\rfloor, \quad N_{r}(j)=\left\lfloor\left(1-R_{\mathrm{in}}\right) / h_{j}+1.5\right\rfloor .
$$

Here, $R_{\text {in }}$ is the inner radius of the boundary-fitted annular grid, $h_{j}=1 /(10 j)$ is the approximate grid spacing for the composite grid with resolution factor $j$, and $\lfloor x\rfloor$ denotes the largest integer less than or equal to $x$. The grid on the left of Figure 11, which we denote by $\mathcal{G}_{D n}^{(j)}$, uses a narrow annular grid with a constant number of grid cells in the radial direction. For this grid, we take $1-R_{\text {in }}=6 h_{j}$ so that $N_{r}=7$ for all $j$. For comparative purposes, we also consider a composite grid which uses an annular grid with a fixed radial width given by $1-R_{\text {in }}=0.25$. This grid, which we denote by $\mathcal{G}_{D f}^{(j)}$, is shown in the middle of Figure 11 .
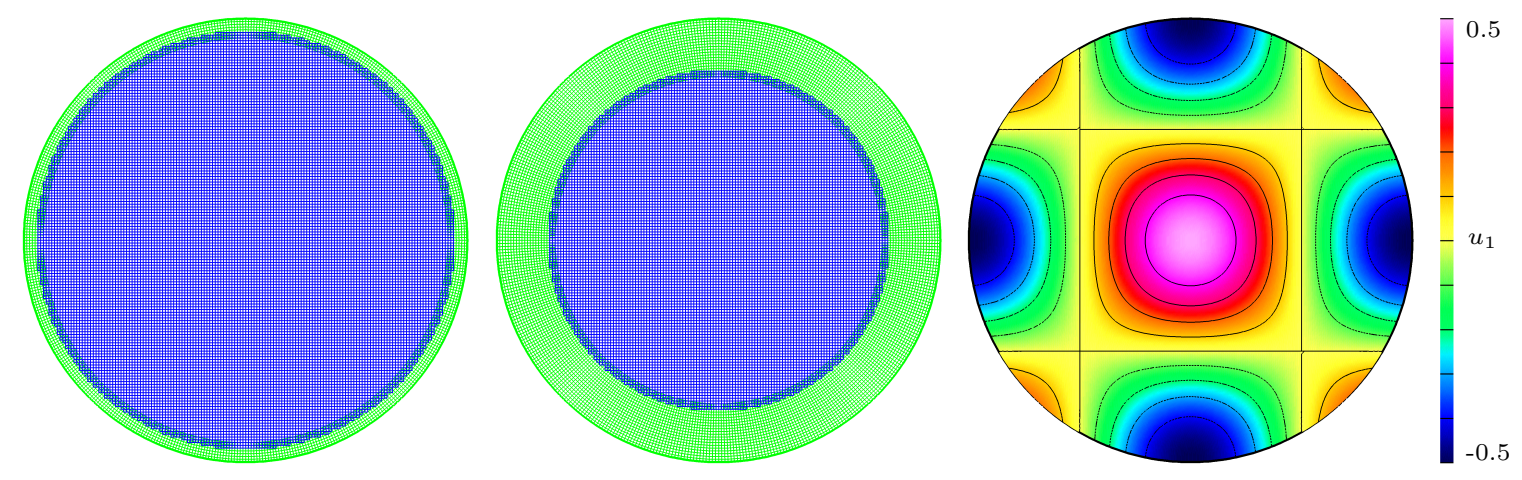

Figure 11: Left: composite grid $\mathcal{G}_{D n}^{(8)}$ for the solid disk (with narrow annulus). Middle: composite grid $\mathcal{G}_{D f}^{(8)}$ for the solid disk (with fixed width annulus). Right: trigonometric solution for $u_{1}$.

To demonstrate the accuracy of the FOS and SOS schemes, the governing equations, with $\rho=\lambda=\mu=1$, are solved on the narrow grids $\mathcal{G}_{D n}^{(j)}, j=2,4,8,16$, to time $t=1.0$. The SOS scheme uses fourth-order dissipation (19) with $\alpha_{4}=1$ and the sixth-order filter (21) with $\beta_{6}=1 / 128$. Figures 12 and 13 give the maximum errors and convergence rates for displacement and traction boundary conditions, respectively. The columns labeled "r" in the figures give the ratio of the error in the current resolution $j$ to that in the previous one $j-1$. These ratios should be approximately equal to four for a second-order accurate scheme. The convergence rates given at the bottom of the figures are computed from a least squares fit to the errors. From the results, both schemes are seen to converge at rates close to second order. We note that the errors in the displacement for the FOS scheme are approximately 10 times smaller that those for the SOS scheme for the case of displacement boundary conditions and approximately 20 times smaller for the case of traction boundary conditions. We will see in subsequent examples that the FOS scheme is generally more accurate than the SOS scheme by a factor of about 10 .

Although the SOS scheme with no dissipation is stable on a single curvilinear grid, our numerical experiments have shown that it is generally unstable on overlapping grids if no dissipation is added. This is in agreement with the stability analysis in Section 5 for the wave equation which showed that a non-dissipative scheme may be unstable on an overlapping grid even though it is stable on a single grid, and that the instability is worse when the grid near the boundary has a constant number of radial cells as the mesh is refined. To examine this, we first solve the elasticity equations using the SOS scheme with no dissipation, and examine the behavior of the error over time. This is done for the choice $\rho=\lambda=\mu=1$ and displacement boundary conditions. Figure 14 shows the error in $u_{1}$ for different grids, as a function of time and as a function of the number of time-steps. (The behavior of the error in the other components of displacement is similar.) Results are shown for the grids $\mathcal{G}_{D n}^{(j)}, j=4,8,16$, which we will refer to as "Case N," and for grids $\mathcal{G}_{D f}^{(j)}, j=4,8,16$, which we refer to as "Case F." We note that the solution is unstable in all cases although the instability can take quite a few time steps to appear. For Case N, the errors grow much more rapidly as 


\begin{tabular}{|c|c|c|c|c|c|c|c|c|c|}
\hline & & \multicolumn{2}{|l|}{ SOS } & \multicolumn{6}{|c|}{ FOS } \\
\hline Grid $\mathcal{G}_{D n}^{(j)}$ & $h_{j}$ & $e_{u}^{(j)}$ & $r$ & $e_{u}^{(j)}$ & $r$ & $e_{v}^{(j)}$ & $r$ & $e_{\sigma}^{(j)}$ & $r$ \\
\hline $\mathcal{G}_{D n}^{(2)}$ & $1 / 20$ & $5.4 \times 10^{-3}$ & & $4.6 \times 10^{-4}$ & & $3.1 \times 10^{-3}$ & & $3.8 \times 10^{-3}$ & \\
\hline $\mathcal{G}_{D n}^{(4)}$ & $1 / 40$ & $1.2 \times 10^{-3}$ & 4.4 & $1.1 \times 10^{-4}$ & 4.3 & $7.0 \times 10^{-4}$ & 4.5 & $9.1 \times 10^{-4}$ & 4.2 \\
\hline $\mathcal{G}_{D n}^{(8)}$ & $1 / 80$ & $3.1 \times 10^{-4}$ & 3.9 & $2.9 \times 10^{-5}$ & 3.7 & $1.7 \times 10^{-4}$ & 4.0 & $2.3 \times 10^{-4}$ & 3.9 \\
\hline $\mathcal{G}_{D n}^{(16)}$ & $1 / 160$ & $7.8 \times 10^{-5}$ & 4.0 & $7.6 \times 10^{-6}$ & 3.8 & $4.3 \times 10^{-5}$ & 4.0 & $5.8 \times 10^{-5}$ & 4.0 \\
\hline \multicolumn{2}{|c|}{ rate } & 2.03 & & 1.97 & & 2.05 & & 2.00 & \\
\hline
\end{tabular}

Figure 12: Elastic disk with displacement boundary conditions and a trigonometric analytic solution: maximum errors and convergence rates at $t=1.0$. The columns labeled " $\mathrm{r}$ " contain the ratio of the error at the current resolution to that at the previous resolution.

\begin{tabular}{|c|c|c|c|c|c|c|c|c|c|}
\hline \multicolumn{2}{|c|}{} & \multicolumn{6}{|c|}{ SOS } & \multicolumn{5}{c|}{ FOS } \\
\hline Grid $\mathcal{G}_{D n}^{(j)}$ & $h_{j}$ & $e_{u}^{(j)}$ & $r$ & $e_{u}^{(j)}$ & $r$ & $e_{v}^{(j)}$ & $r$ & $e_{\sigma}^{(j)}$ & $r$ \\
\hline $\mathcal{G}_{D n}^{(2)}$ & $1 / 20$ & $8.4 \times 10^{-3}$ & & $1.4 \times 10^{-3}$ & & $3.3 \times 10^{-3}$ & & $2.1 \times 10^{-2}$ & \\
\hline $\mathcal{G}_{D n}^{(4)}$ & $1 / 40$ & $2.9 \times 10^{-3}$ & 3.1 & $2.3 \times 10^{-4}$ & 6.1 & $5.8 \times 10^{-4}$ & 5.6 & $5.3 \times 10^{-3}$ & 4.0 \\
\hline $\mathcal{G}_{D n}^{(8)}$ & $1 / 80$ & $8.0 \times 10^{-4}$ & 3.6 & $4.7 \times 10^{-5}$ & 4.8 & $1.3 \times 10^{-4}$ & 4.6 & $1.3 \times 10^{-3}$ & 4.0 \\
\hline $\mathcal{G}_{D n}^{(16)}$ & $1 / 160$ & $2.1 \times 10^{-4}$ & 3.9 & $1.1 \times 10^{-5}$ & 4.3 & $2.9 \times 10^{-5}$ & 4.3 & $3.4 \times 10^{-4}$ & 3.9 \\
\hline \multicolumn{2}{|c|}{ rate } & 1.82 & & 2.32 & & 2.27 & & 1.99 & \\
\hline
\end{tabular}

Figure 13: Elastic disk with traction boundary conditions and a trigonometric analytic solution: maximum errors and convergence rates at $t=1.0$. The columns labeled " $\mathrm{r}$ " contain the ratio of the error at the current resolution to that at the previous resolution.

the grids are refined, while for Case $\mathrm{F}$ the errors only show a relatively modest growth, even though many more time steps are being taken on the finer grids. Case $\mathrm{N}$ is the more difficult case, and its solutions are behaving as if there is an unstable mode with amplification factor per time step equal to $\mathcal{A} \approx e^{\alpha} \approx 1+\alpha$, for some small $\alpha>0$, which does not depend on $h_{j}$. In this case the errors would grow to a certain large value after a fixed number of time steps. Solutions for Case F, on the other hand, appear to have an unstable mode with amplification equal to $\mathcal{A} \approx e^{\alpha h_{j}^{\gamma}} \approx 1+\alpha h_{j}^{\gamma}$, for some $\gamma$ with $0<\gamma<1$, and thus the instability becomes less severe as the mesh is refined. This is indicated in the figure by the fact that many more time steps are needed before the instability appears. These results are entirely consistent with our analytical results for the wave equation.

The analysis in Section 5 also showed how the addition of an artificial dissipation or a filter should be able to stabilize the SOS scheme provided the dissipation or filter is chosen in an appropriate way. The Godunov scheme for the first-order wave equation has a built in high-order dissipation, and it was shown how this dissipation could stabilize the FOS scheme. To study this behavior, we consider a long-time integration of the governing equations on the grid $\mathcal{G}_{D n}^{(4)}$ using the SOS scheme with the fourth-order dissipation and sixth-order filter defined previously, and using the FOS scheme. The results are shown in Figure 14. Both schemes remain stable to the final time $t=1000$, which required 130,800 time steps for the SOS scheme and 160,600 time steps for the FOS scheme. The errors in both cases oscillate about some fixed mean with no indication of any systematic growth over time. We also note that although both schemes have some dissipation, the solutions do not decay to zero since we are solving the forced equations.

\subsection{Spherical cavity in a box}

In this next case, we consider a three-dimensional domain consisting of a spherical cavity in a box, and apply the method of analytic solutions as discussed in Appendix C using trigonometric functions for the components of displacement, velocity and stress given by

$$
\begin{aligned}
& \bar{u}_{1}=c_{1} c_{2} c_{3} c_{t}, \quad \bar{u}_{2}=.5 c_{1} s_{2} c_{3} c_{t}, \quad \bar{u}_{3}=.75 c_{1} c_{2} s_{3} c_{t}, \\
& \bar{v}_{1}=.75 s_{1} c_{2} c_{3} c_{t}, \bar{v}_{2}=.25 c_{1} c_{2} s_{3} c_{t}, \quad \bar{v}_{3}=-.5 s_{1} s_{2} s_{3} c_{t}, \\
& \bar{\sigma}_{11}=-.5 c_{1} c_{2} c_{3} c_{t}, \quad \bar{\sigma}_{12}=.4 s_{1} c_{2} c_{3} c_{t}, \quad \bar{\sigma}_{13}=.6 c_{1} c_{2} s_{3} c_{t}, \\
& \bar{\sigma}_{21}=.4 s_{1} c_{2} c_{3} c_{t}, \quad \bar{\sigma}_{22}=-.7 s_{1} c_{2} s_{3} c_{t}, \quad \bar{\sigma}_{23}=.65 c_{1} s_{2} s_{3} c_{t}, \\
& \bar{\sigma}_{31}=.6 c_{1} c_{2} s_{3} c_{t}, \quad \bar{\sigma}_{32}=.65 c_{1} s_{2} s_{3} c_{t}, \quad \bar{\sigma}_{33}=-.2 s_{1} s_{2} s_{3} c_{t},
\end{aligned}
$$



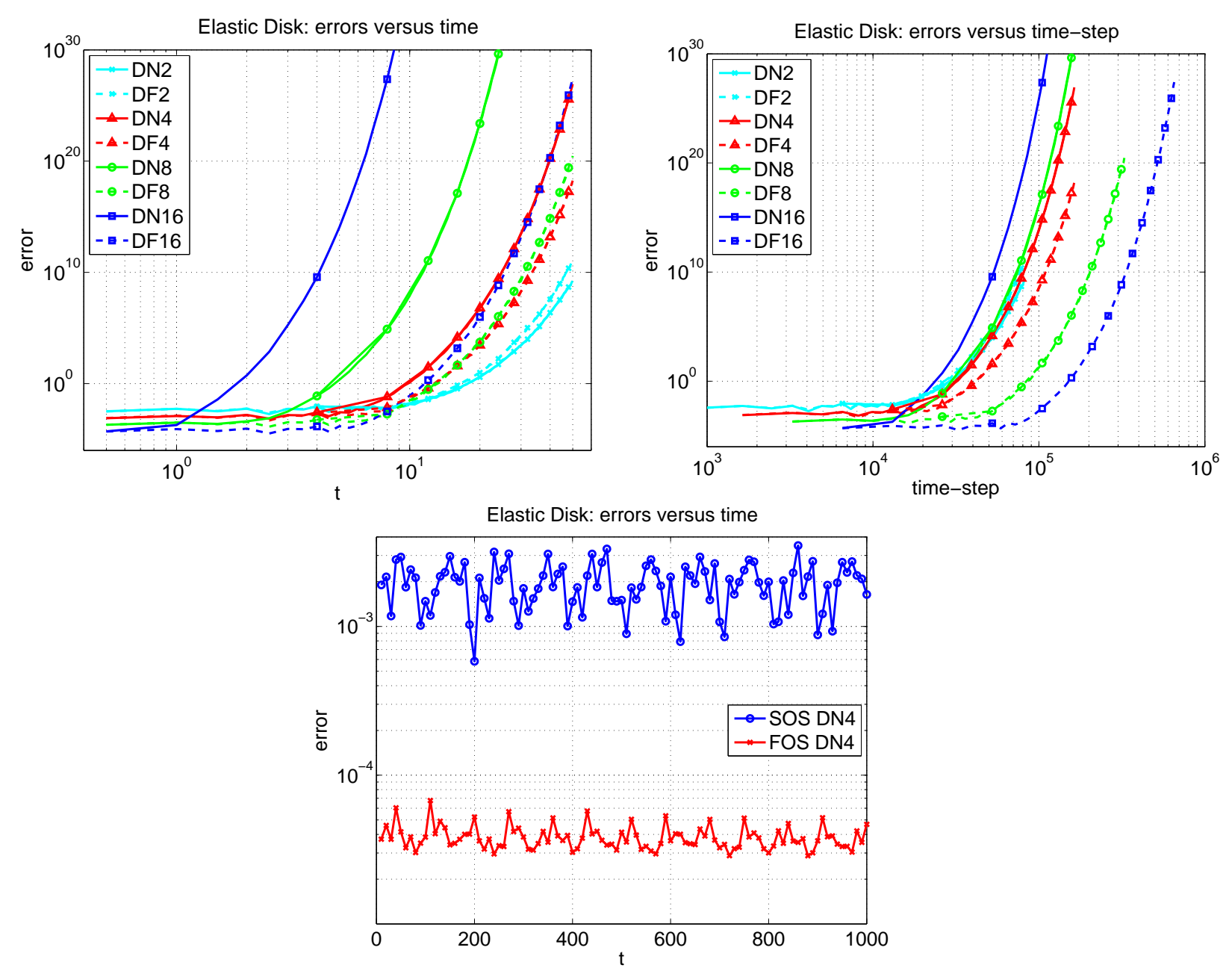

Figure 14: Top left and right: growth of the errors in $u_{1}$ versus time and versus the number of time-steps for the SOS scheme without dissipation on the solid disk domain. The instability is caused by the overlap boundary between grids. Results are shown for narrow grids $\mathcal{G}_{D n}^{(j)}, j=2,4,8,16$ (labeled DN2, DN4, etc.) and fixed grids $\mathcal{G}_{D f}^{(j)}, j=2,4,8,16$ (labeled DF2, DF4, etc.) Bottom: errors in $u_{1}$ for the stabilized SOS scheme and the FOS scheme for $t \in[0,1000]$. Both schemes are stable to the final time $t=1000$ (approximately 150,000 time steps).

where now $\left(c_{j}, s_{j}\right)=\left(\cos \left(\pi x_{j} / 2\right), \sin \left(\pi x_{j} / 2\right)\right), j=1,2,3$, and $c_{t}=\cos (\pi t / 2)$. The composite grid for this domain is shown in Figure 15, and it consists of a Cartesian background grid of length 2.4 on each side and two orthographic patches. The orthographic patches cut a hole in the background grid and define the boundary of the spherical cavity of radius 0.5 as shown in the figure. The box grid is defined as

$$
\begin{aligned}
\mathcal{B}\left(\left[x_{a}, x_{b}\right] \times\left[y_{a}, y_{b}\right] \times\left[z_{a}, z_{b}\right], N_{1}, N_{2}, N_{3}\right)=\left\{\left(x_{a}+i_{1} \Delta x, y_{a}+i_{2} \Delta y, z_{a}+i_{3} \Delta z\right) \mid\right. \\
\left.\quad \Delta x=\left(x_{b}-x_{a}\right) / N_{1}, \Delta y=\left(y_{b}-y_{a}\right) / N_{2}, \Delta z=\left(z_{b}-z_{a}\right) / N_{3}, i_{\alpha}=0,1, \ldots, N_{\alpha}, \alpha=1,2,3\right\} .
\end{aligned}
$$

The orthographic grids are defined in terms of the orthographic transform $\mathbf{O}_{p}$, given by

$$
\mathbf{x}=\mathbf{O}_{p}\left(\mathbf{r} ;\left[\varrho_{a}, \varrho_{b}\right], \hat{s}_{2}, \hat{s}_{3}\right) \equiv\left(p \frac{\left(1-\sigma^{2}\right) \varrho}{1+\sigma^{2}}, \frac{2 \varrho s_{2}}{1+\sigma^{2}}, p \frac{2 \varrho s_{3}}{1+\sigma^{2}}\right)
$$

where $\varrho, s_{2}, s_{3}$ and $\sigma$ are given in terms of $\mathbf{r}=\left(r_{1}, r_{2}, r_{3}\right) \in[0,1]^{3}$ by

$$
\varrho=\varrho_{a}+r_{1}\left(\varrho_{b}-\varrho_{a}\right), \quad s_{2}=\left(r_{2}-\frac{1}{2}\right) \hat{s}_{2}, \quad s_{3}=\left(r_{3}-\frac{1}{2}\right) \hat{s}_{3}, \quad \sigma^{2}=s_{2}^{2}+s_{3}^{2},
$$

and $p=+1$ for the transformation near the north pole and $p=-1$ for the transformation near the south pole. The parameters $\left[\varrho_{a}, \varrho_{b}\right]$ specify the radial extent of the region, while $\hat{s}_{2}$ and $\hat{s}_{3}$ determine its lateral 
extent. The orthographic grid, $\mathcal{O}_{p}$ centered about pole $p$, is now defined as

$$
\mathcal{O}_{p}\left(\left[\varrho_{a}, \varrho_{b}\right], \hat{s}_{2}, \hat{s}_{3}, N_{1}, N_{2}, N_{3}\right)=\left\{\mathbf{x}_{\mathbf{i}} \mid \mathbf{x}_{\mathbf{i}}=\mathbf{O}_{p}\left(\mathbf{r}_{\mathbf{i}} ;\left[\varrho_{a}, \varrho_{b}\right], \hat{s}_{2}, \hat{s}_{3}\right), i_{\alpha}=0,1, \ldots, N_{\alpha}, \alpha=1,2,3\right\} .
$$

The number of grid points in each coordinate direction for a grid with resolution factor $j$ is chosen so that the grid spacing is approximately $h_{j}=1 /(5 j)$. The sphere-in-a-box composite grid is then defined as

$$
\mathcal{G}_{\mathrm{s}}^{(j)}=\mathcal{B}\left([-1.2,1.2]^{3}, N_{x}(j), N_{x}(j), N_{x}(j)\right) \cup \mathcal{O}_{ \pm 1}\left([.5, .9], 2.1,2.1, N_{r}(j), N_{o}(j), N_{o}(j)\right),
$$

where $N_{x}(j)=\left\lfloor 2.4 / h_{j}+1.5\right\rfloor, N_{r}(j)=\left\lfloor 0.4 / h_{j}+1.5\right\rfloor$ and $N_{o}(j)=\left\lfloor 2.24 / h_{j}+1.5\right\rfloor$.
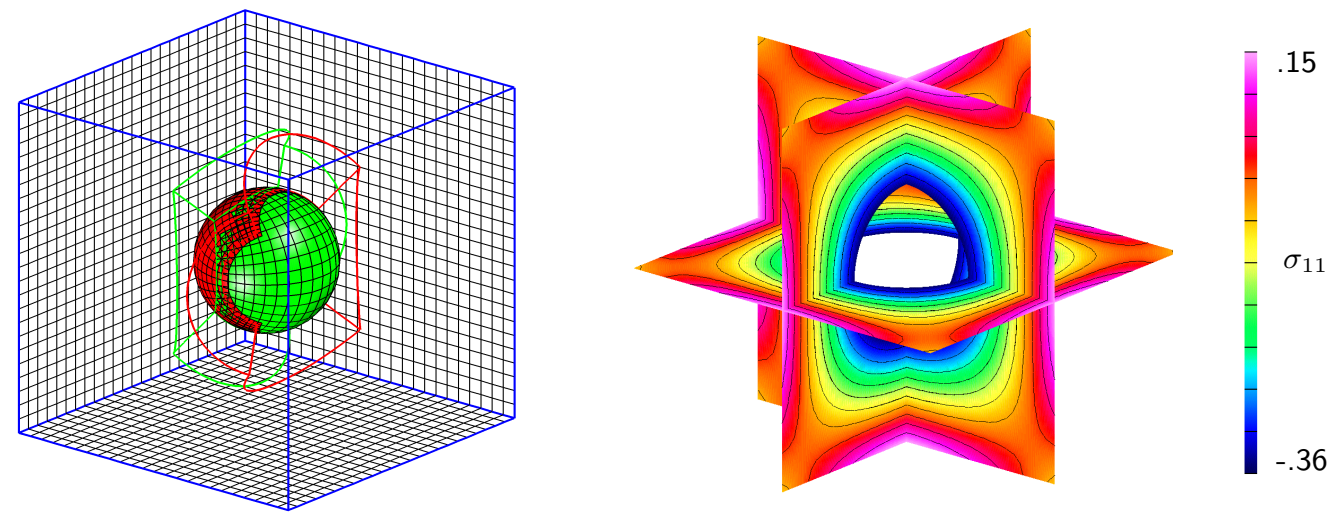

Figure 15: Spherical cavity in a box with a trigonometric solution: grid $\mathcal{G}_{\mathrm{s}}^{(2)}$ (left) and solution for $\sigma_{11}$ at $t=0.1$ from the FOS scheme (right).

The errors in the various components using the SOS and FOS schemes with $\mathcal{G}_{\mathrm{s}}^{(j)}, j=2,4,8$, are given in Figure 16 for the case of displacement boundary conditions, while the corresponding results for the case of traction boundary conditions are given in Figure 17. A representative component of the solution at $t=0.1$ is shown in Figure 15. The finest grid used, $\mathcal{G}_{\mathrm{s}}^{(8)}$, has about one million grid points, and the calculations were performed in parallel on 8 processors. The convergence rates for the SOS and FOS schemes are reasonably close to 2. We note also that some of the variables for the FOS calculation seem to be converging at a rate

\begin{tabular}{|c|c|c|c|c|c|c|c|c|c|}
\hline & & \multicolumn{2}{|c|}{ SOS } & \multicolumn{6}{|c|}{ FOS } \\
\hline Grid $\mathcal{G}_{\mathrm{s}}^{(j)}$ & $h_{j}$ & $e_{u}^{(j)}$ & $r$ & $e_{u}^{(j)}$ & $r$ & $e_{v}^{(j)}$ & $r$ & $e_{\sigma}^{(j)}$ & $r$ \\
\hline $\mathcal{G}_{\mathrm{s}}^{(2)}$ & $1 / 10$ & $7.1 \times 10^{-3}$ & & $1.5 \times 10^{-3}$ & & $2.9 \times 10^{-3}$ & & $1.5 \times 10^{-2}$ & \\
\hline $\mathcal{G}_{\mathrm{s}}^{(4)}$ & $1 / 20$ & $6.6 \times 10^{-4}$ & 10.7 & $2.5 \times 10^{-4}$ & 6.0 & $4.3 \times 10^{-4}$ & 6.7 & $3.7 \times 10^{-3}$ & 4.1 \\
\hline $\mathcal{G}_{\mathrm{s}}^{(8)}$ & $1 / 40$ & $1.4 \times 10^{-4}$ & 4.7 & $3.6 \times 10^{-5}$ & 7.0 & $1.0 \times 10^{-4}$ & 4.2 & $9.1 \times 10^{-4}$ & 4.0 \\
\hline \multicolumn{2}{|c|}{ rate } & 2.82 & & 2.70 & & 2.41 & & 2.02 & \\
\hline
\end{tabular}
larger than 2, but this is likely caused by the grids being still relatively coarse.

Figure 16: Spherical cavity in a box with a trigonometric analytic solution: maximum errors and convergence rates at $t=0.1$ for displacement boundary conditions. The columns labeled " $\mathrm{r}$ " contain the ratio of the error at the current resolution to that at the previous resolution.

\begin{tabular}{|c|c|c|c|c|c|c|c|c|c|}
\hline \multicolumn{2}{|c|}{} & \multicolumn{2}{|c|}{ SOS } & \multicolumn{7}{c|}{ FOS } \\
\hline Grid $\mathcal{G}_{\mathrm{s}}^{(j)}$ & $h_{j}$ & $e_{u}^{(j)}$ & $r$ & $e_{u}^{(j)}$ & $r$ & $e_{v}^{(j)}$ & $r$ & $e_{\sigma}^{(j)}$ & $r$ \\
\hline $\mathcal{G}_{\mathrm{s}}^{(2)}$ & $1 / 10$ & $8.3 \times 10^{-3}$ & & $1.5 \times 10^{-3}$ & & $7.8 \times 10^{-3}$ & & $2.1 \times 10^{-2}$ & \\
\hline $\mathcal{G}_{\mathrm{s}}^{(4)}$ & $1 / 20$ & $2.0 \times 10^{-3}$ & 4.2 & $2.5 \times 10^{-4}$ & 6.0 & $1.0 \times 10^{-3}$ & 7.6 & $5.2 \times 10^{-3}$ & 4.1 \\
\hline $\mathcal{G}_{\mathrm{s}}^{(8)}$ & $1 / 40$ & $4.9 \times 10^{-4}$ & 4.0 & $3.6 \times 10^{-5}$ & 7.0 & $2.3 \times 10^{-4}$ & 4.4 & $1.3 \times 10^{-3}$ & 4.1 \\
\hline \multicolumn{2}{|c|}{ rate } & 2.04 & & 2.70 & & 2.53 & & 2.03 & \\
\hline
\end{tabular}

Figure 17: Spherical cavity in a box with a trigonometric analytic solution: maximum errors and convergence rates at $t=0.1$ for traction boundary conditions. The columns labeled " $r$ " contain the ratio of the error at the current resolution to that at the previous resolution. 


\subsection{Traveling pulse with AMR}

In this section, we consider the accuracy of the AMR option described in Section 3.2 using the method of analytic solutions. To do this, we consider a traveling pulse function of the form

$$
\mathcal{P}(\mathbf{x}, t)=\exp \left\{-\left(\left|\mathbf{x}-\mathbf{x}_{c}(t)\right| / L\right)^{2}\right\}
$$

where $L$ measures the width of the pulse and $\mathbf{x}_{c}(t)$ gives the position of the center of the pulse at a time $t$. We set $\mathbf{x}_{c}(t)=\mathbf{x}_{0}+\mathbf{v}_{0} t$, where $\mathbf{x}_{0}$ gives the position of the center of the pulse at $t=0$ and $\mathbf{v}_{0}$ gives its constant velocity. For an IBVP on a square domain $\Omega_{S}=[-1,1]^{2}$ with displacement boundary conditions, we apply the method of analytic solutions by setting $\overline{\mathbf{u}}(\mathbf{x}, t), \overline{\mathbf{v}}(\mathbf{x}, t)$ and $\overline{\boldsymbol{\sigma}}(\mathbf{x}, t)$ equal to the pulse function $\mathcal{P}(\mathbf{x}, t)$ with $L=1 / 30, \mathbf{x}_{0}=(-0.5,-0.5)$ and $\mathbf{v}_{0}=(1,1)$. We note that since $L$ is small on the scale of $\Omega_{S}$, the pulse is localized in space at any given time. Thus, refinement grids are created about the pulse and AMR structure evolves in time to resolve the solution. In this way, the behavior and accuracy of the AMR implementation is checked.
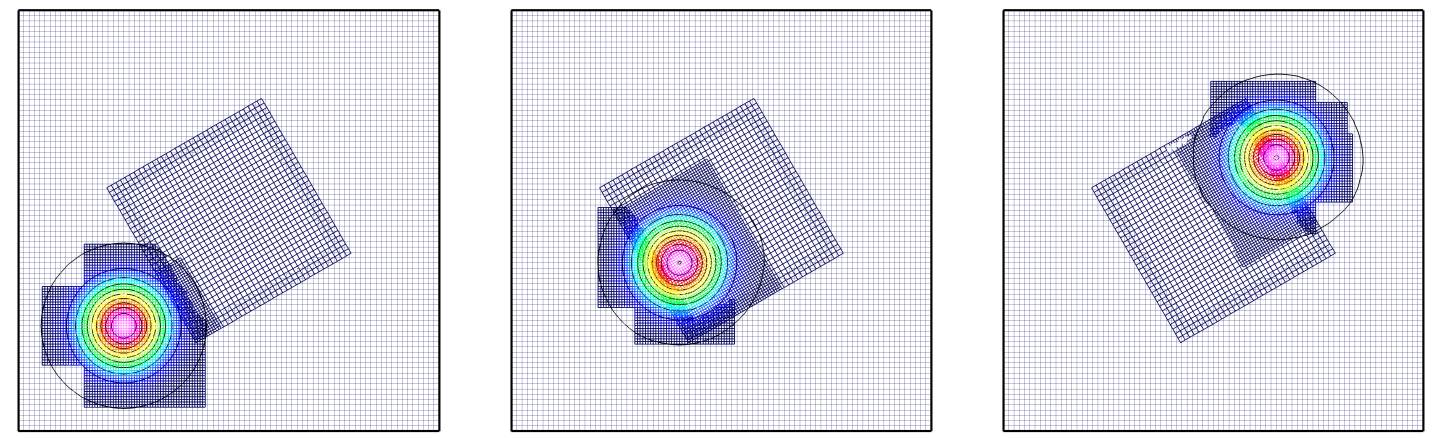

Figure 18: Propagation of a pulse through an embedded rotated grid, computed using AMR and the SOS scheme. The locations of the refinement grids are recomputed every 4 time-steps. Contours of the displacement $u_{1}$ at times $t=0$ (left), $t=0.3$ (middle), and $t=0.8$ (right), are plotted so that the grids are also shown.

Numerical calculations of the IBVP are performed using a rotated-square-in-a-square grid, $\mathcal{G}_{R S}^{(j)}$, defined by the union of a background Cartesian grid for the square $[-1,1]^{2}$ together with an embedded Cartesian grid for the domain $[-0.4,0.4]^{2}$ which is rotated by $30^{\circ}$ about its center. The purpose of the embedded grid is to illustrate the behavior of the AMR as a moving localized feature passes through a grid overlap, and to test the accuracy of the results for this situation. The grid spacing for $\mathcal{G}_{R S}^{(j)}$ is $h_{j}=1 /(10 j)$ and one refinement level is used with refinement factor $n_{r}=2$. The parameters in the AMR error estimate (10) are chosen as $c_{1}=1, c_{2}=1$ and $s_{k}=1$. An error tolerance of $10^{-4}$ is used for the SOS scheme and $10^{-3}$ for the FOS scheme. The error is smoothed once, the number of buffer zones is 2 and the AMR grid is regenerated every 4 time steps.

Figure 18 shows contours of the first component of displacement and the corresponding AMR grids at three different times using the SOS scheme. The corresponding results for the FOS scheme are nearly indistinguishable from those given by the SOS scheme and thus are not shown. We observe that the pulse propgates cleanly through the grid overlap with no visible distortion or spurious reflections. The maximum errors and computed convergence rates for this problem are given in Figure 19. The results indicate that the solutions given by the SOS and FOS schemes converge at second-order accuracy when AMR is used on an overlapping grid.

\begin{tabular}{|c|c|c|c|c|c|c|c|c|c|}
\hline \multicolumn{2}{|c|}{} & \multicolumn{2}{|c|}{ SOS } & \multicolumn{5}{c|}{ FOS } \\
\hline Grid $\mathcal{G}_{R S}^{(j)}$ & $h_{j}$ & $e_{u}^{(j)}$ & $r$ & $e_{u}^{(j)}$ & $r$ & $e_{v}^{(j)}$ & $r$ & $e_{\sigma}^{(j)}$ & $r$ \\
\hline $\mathcal{G}_{R S}^{(1)}$ & $1 / 10$ & $3.7 \times 10^{-2}$ & & $5.6 \times 10^{-3}$ & & $2.2 \times 10^{-2}$ & & $4.3 \times 10^{-2}$ & \\
\hline $\mathcal{G}_{R S}^{(2)}$ & $1 / 20$ & $6.1 \times 10^{-3}$ & 6.1 & $1.4 \times 10^{-3}$ & 3.8 & $5.3 \times 10^{-3}$ & 4.2 & $1.1 \times 10^{-2}$ & 3.9 \\
\hline $\mathcal{G}_{R S}^{(4)}$ & $1 / 40$ & $1.5 \times 10^{-3}$ & 4.0 & $3.7 \times 10^{-4}$ & 3.9 & $1.4 \times 10^{-3}$ & 3.7 & $2.5 \times 10^{-3}$ & 4.3 \\
\hline $\mathcal{G}_{R S}^{(8)}$ & $1 / 80$ & $3.9 \times 10^{-4}$ & 4.0 & $6.8 \times 10^{-5}$ & 5.4 & $3.6 \times 10^{-4}$ & 4.0 & $6.3 \times 10^{-4}$ & 4.0 \\
\hline \multicolumn{2}{|c|}{ rate } & 2.18 & & 2.11 & & 1.97 & & 2.03 & \\
\hline
\end{tabular}

Figure 19: Traveling pulse with AMR on a rotated-square-in-a-square grid. The maximum errors and computed convergence rates at $t=1.0$ are given for the SOS and FOS schemes. 


\subsection{Vibrational modes of an elastic annulus}

In this section and the next, we apply the SOS and FOS schemes to IBVPs for which exact solutions exist (without applying artificial forcing functions to construct solutions). As a first case, we consider a two-dimensional annular domain $\Omega_{A}$ with inner radius $R_{a}$ and outer radius $R_{b}$. Analytic solutions for this domain are given in Appendix D.1. To evaluate the accuracy of the numerical schemes, we consider an exact solution that consists of a superposition of the time-independent solution in (D.4) and the first vibrational mode in (D.6), i.e.

$$
u_{r}=A_{0} r+\frac{B_{0}}{r}+\left[\left(A_{1} J_{1}\left(\alpha_{1} r\right)+B_{1} Y_{1}\left(\alpha_{1} r\right)\right] \cos \left(\omega_{1} t\right) .\right.
$$

We consider four cases with boundary conditions and parameters given in Figure 20. For all cases, we assume that $\rho=\mu=1$ and that $R_{a}=1 / 2$ and $R_{b}=1$. Values for $\alpha_{1}, A_{1}$ and $B_{1}$ are given in the figure for each choice of the boundary conditions and the value for $\lambda$, whereas $A_{0}$ and $B_{0}$ are equal to zero for the case of displacement boundary conditions and are given by (D.5) for the case of traction boundary conditions with the choice of $\left(P_{a}, P_{b}\right)$ given in the figure. To scale the displacements to be of size one approximately, we let $A_{1}=C H_{1}\left(\alpha_{1} R_{a}\right)$ and $B_{1}=-C G_{1}\left(\alpha_{1} R_{a}\right)$, and set $C=5$ for the case of displacement boundary conditions and $C=1 / 10$ for the case of traction boundary conditions.

\begin{tabular}{|c|c|c|c|c|c|c|}
\hline Case & $\mathrm{BC}$ & $\lambda$ & $\left(P_{a}, P_{b}\right)$ & $\alpha_{1}$ & $A_{1}$ & $B_{1}$ \\
\hline 1 & $\mathrm{D}$ & 1 & - & 6.3931567616 & 1.8502739846 & -1.3135880300 \\
2 & $\mathrm{~T}$ & 1 & $(1,2)$ & 1.3113530190 & 0.1861924685 & -0.1140163754 \\
3 & $\mathrm{D}$ & 100 & - & 6.3931567616 & 1.8502739846 & -1.3135880300 \\
4 & $\mathrm{~T}$ & 100 & $(1,2)$ & 6.2525010635 & 1.7624191636 & 1.6031685352 \\
\hline
\end{tabular}

Figure 20: Solution parameters for the first vibrational mode in an annulus for displacement boundary conditions (D) or traction boundary conditions $(\mathrm{T})$.

Numerical solutions are computed using a family of composite grids for the annulus $\Omega_{A}$ each consisting of a single annular grid defined in (74). The composite grids with resolution factor $j$ are defined by

$$
\mathcal{G}_{A}^{(j)}=\mathcal{C}\left(\left[R_{a}, R_{b}\right], N_{\theta}(j), N_{r}(j)\right),
$$

where $N_{r}(j)=\left\lfloor .5 / h_{j}+2.5\right\rfloor, N_{\theta}(j)=\left\lfloor 2 \pi(.75) / h_{j}+1.5\right\rfloor$, and the grid spacing is $h_{j}=1 /(10 j)$. Initial conditions for displacement and velocity are taken from the exact solution in (79). Figure 21 shows shaded contours of various components of stress at different times computed using the FOS scheme on grid $\mathcal{G}_{A}^{(4)}$ for the case of traction boundary conditions and $\lambda=1$ (Case 2). We observe that the solution varies smoothly on the grid and has symmetry in agreement with the fact that $u_{r}=u_{r}(r, t)$ and the displacement in the $\theta$ direction is zero.
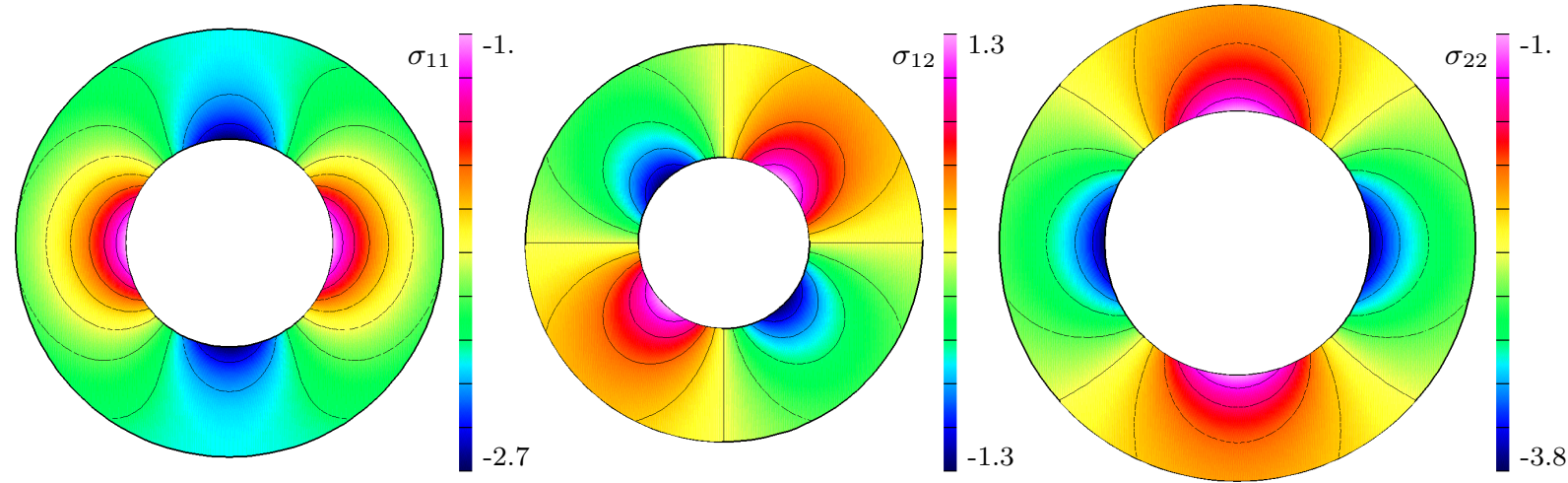

Figure 21: Vibrational mode of an elastic annulus with traction boundary conditions (Case 2). Shaded contours of components of the stress at various times plotted on the deformed grid, scaling the displacement by a factor of $0.25: \sigma_{11}$ at $t=0$ (left), $\sigma_{12}$ at $t=0.6$ (middle) and $\sigma_{22}$ at $t=2.0$ (right).

Figure 22 shows the behavior of the error in the displacement for the SOS scheme and the error in the displacement, velocity and stress for the FOS scheme for Cases 1 and 2. We note that the SOS scheme uses the sixth-order filter (with $\beta_{6}=1 / 128$ ) to be consistent with the SOS calculations in other sections, even though it is not necessary for the single-grid calculations for this problem. The black line in each plot has a slope equal to two which is the theoretical convergence rate for the schemes. The general behavior of the 

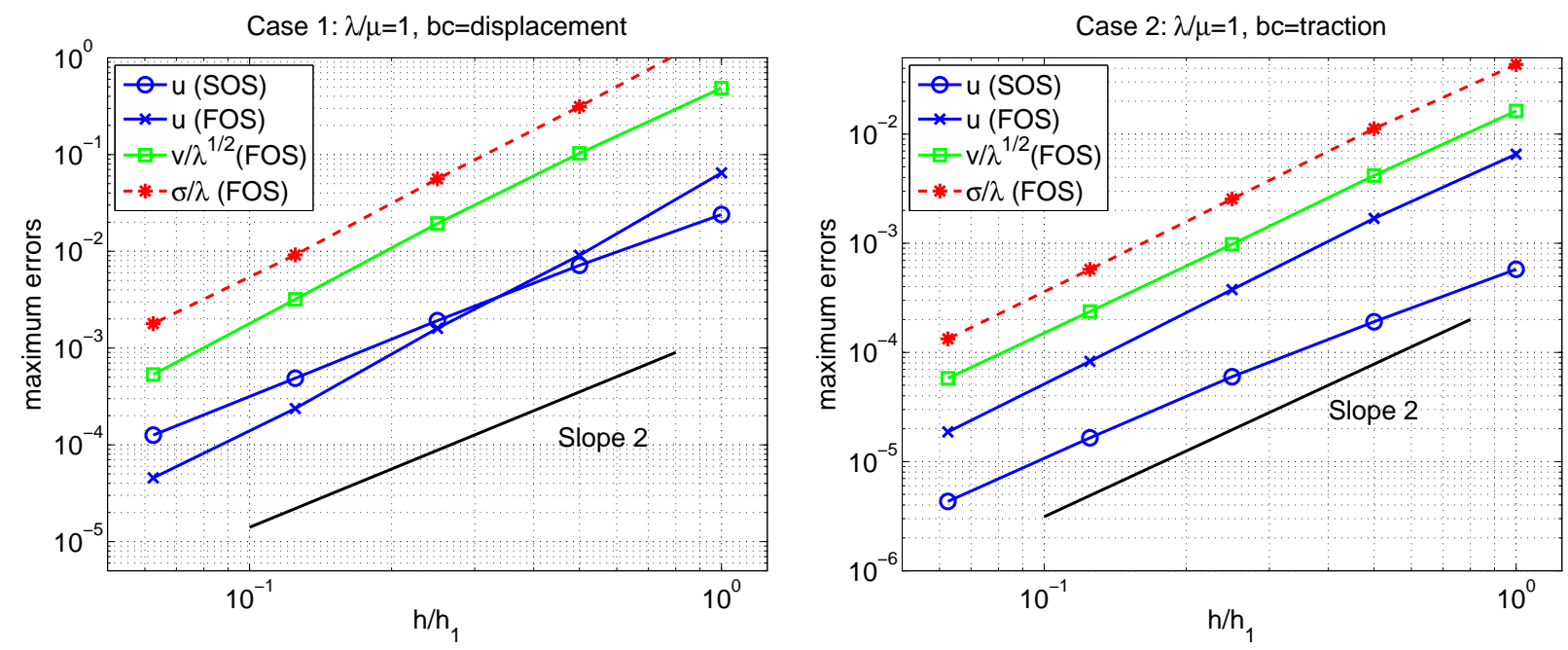

Figure 22: Vibrational mode of an annulus. Maximum errors at $t=0.5$ for Case 1 (left) and Case 2 (right).

errors is to approach a slope of two as the mesh spacing becomes smaller in agreement with the expected convergence rate.

Figure 22 shows the behavior of the error in the displacement for the SOS scheme and the error in the displacement, velocity and stress for the FOS scheme for Cases 3 and 4 . These cases with $\lambda=100$ are more difficult since many more time steps are required to reach the final time and the traction boundary conditions for Case 4 can be sensitive when $\lambda / \mu$ is large. Despite these difficulties, the convergence rates shown in the plots are close to two (and in some cases better than two).
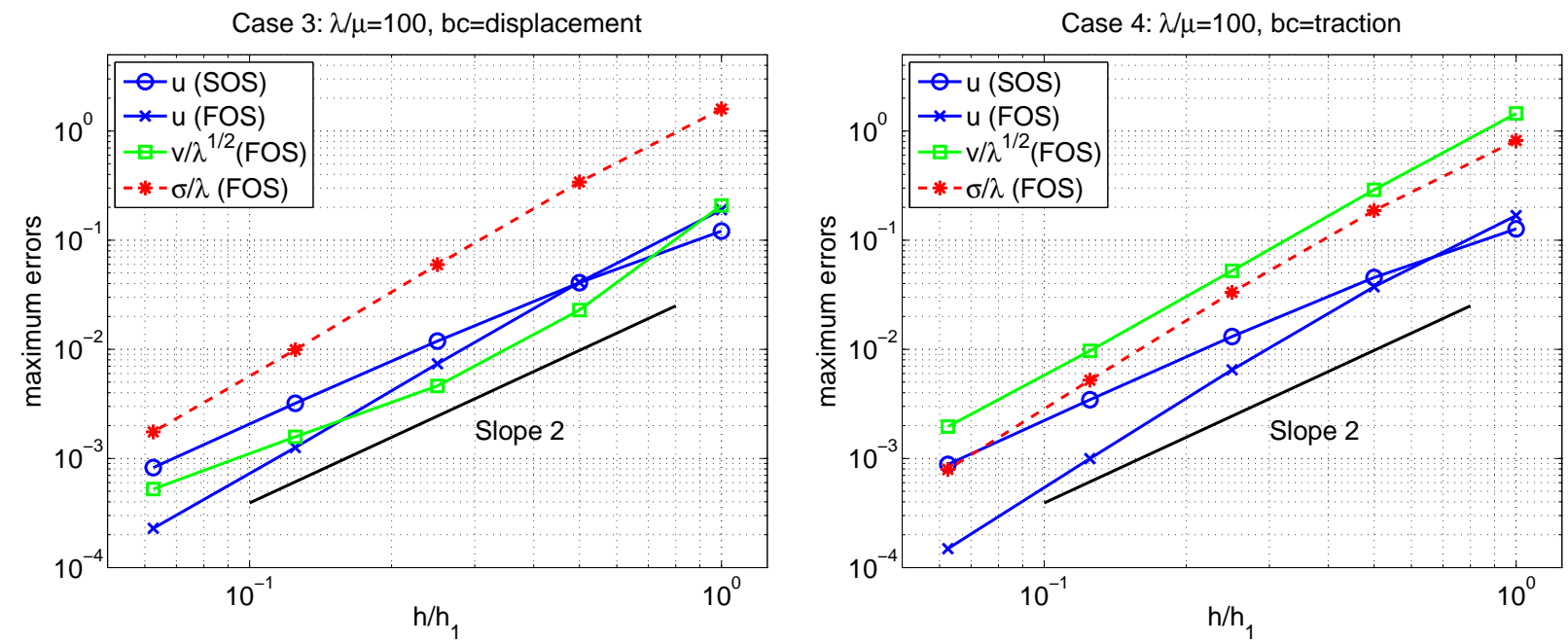

Figure 23: Vibrational mode of an annulus. Maximum errors at $t=0.5$ for Case 3 (left) and Case 4 (right). The errors for $\mathbf{v}$ and $\boldsymbol{\sigma}$ are scaled by $\lambda$ for illustrative purposes.

\subsection{Vibrational modes of an elastic sphere}

In this section, we consider small amplitude vibrations of a solid elastic sphere. Modes of vibration of the solid sphere, described in Appendix D.2, may be computed numerically using the overlapping grid shown in Figure 24. The overlapping grid, shown in the left-most image in the figure, is defined by four component grids, three of which are curvilinear and define the spherical boundary as shown in the figure. The fourth component grid is a Cartesian grid which covers the interior core of the solid sphere and is not visible in the figure. Most of the boundary-fitted spherical shell is covered by a spherical-polar grid defined by

$$
\begin{gathered}
\mathcal{S}\left(\left[\varrho_{a}, \varrho_{b}\right] \times\left[\theta_{a}, \theta_{b}\right] \times\left[\phi_{a}, \phi_{b}\right], N_{1}, N_{2}, N_{3}\right)=\left\{\left(\varrho_{i_{1}} \cos \theta_{i_{2}} \sin \phi_{i_{3}}, \varrho_{i_{1}} \sin \theta_{i_{2}} \sin \phi_{i_{3}}, \varrho_{i_{1}} \cos \phi_{i_{3}}\right) \mid\right. \\
\varrho_{i_{1}}=\varrho_{a}+i_{1}\left(\varrho_{b}-\varrho_{a}\right) / N_{1}, \theta_{i_{2}}=\theta_{a}+i_{2}\left(\theta_{b}-\theta_{a}\right) / N_{2}, \phi_{i_{3}}=\phi_{a}+i_{3}\left(\phi_{b}-\phi_{a}\right) / N_{3}, \\
\left.i_{\alpha}=0,1, \ldots, N_{\alpha}, \alpha=1,2,3\right\} .
\end{gathered}
$$


The parts of the spherical shell near the north and south poles are covered by orthographic patches defined in (78), and the Cartesian grid in the interior is defined by the box grid in (77). The overlapping grid for the solid sphere of radius $R$, with resolution factor $j$, is given by

$$
\begin{aligned}
\mathcal{G}_{\mathrm{ss}}^{(j)}=\mathcal{B}\left(\left[-x_{a}, x_{a}\right]^{3}, N_{x}(j), N_{x}(j), N_{x}(j)\right) & \cup \mathcal{S}\left([.75 R, R] \times[0,2 \pi] \times[.2 \pi, .8 \pi], N_{r}(j), N_{\theta}(j), N_{\phi}(j)\right) \\
& \cup \mathcal{O}_{ \pm 1}\left([.75 R, R], S_{a}, S_{a}, N_{r}(j), N_{0}(j), N_{0}(j)\right),
\end{aligned}
$$

where

$$
\begin{gathered}
x_{a}=.75 R+1.5 h_{j}, \quad N_{x}(j)=\left\lfloor 2 x_{a} / h_{j}\right\rfloor, \quad S_{a}=.65+h_{j} / R, \quad N_{0}(j)=\left\lfloor .49 S_{a} \pi R / h_{j}+1.5\right\rfloor, \\
N_{r}(j)=\left\lfloor .25 R / h_{j}+1.5\right\rfloor, \quad N_{\theta}(j)=\left\lfloor 1.4 \pi R / h_{j}+1.5\right\rfloor, \quad N_{\phi}(j)=\left\lfloor .51 R \pi / h_{j}+1.5\right\rfloor,
\end{gathered}
$$

for a mesh spacing $h_{j}=R /(10 j)$. We note that the grid spacings for each component grid are approximately the same. This is done in order to improve the accuracy of the solution near interpolation boundaries. It also means that the global time step is close to the maximum allowable time step for each component grid. As an example, for grid $\mathcal{G}_{\mathrm{ss}}^{(8)}$, the global time step is a factor of 0.8 smaller than the largest allowable time step for any component grid.

Using the exact solution with the first $\left(\kappa_{2}, C_{2}\right)$ pair in Figure D.36 to obtain initial conditions, numerical solutions are computed using the SOS and FOS schemes for the case $\rho=\lambda=\mu=1, R=1$ and $A_{2}=100$. (The amplitude is chosen so that the maximum displacement is about 1 in magnitude.) The deformation of the sphere is shown in the left-middle, right-middle and right-most images in Figure 24 for $t=0,0.8$ and 1.2, respectively. The maximum error between the various components of the numerical solution and the exact solution at $t=0.5$ is given in Figure 25 for four grid resolutions. The computed rates given in the figure indicate that the numerical solutions given by the two schemes are both converging at a rate approximately equal to 2. Figure 26 shows the maximum errors in $\mathbf{u}$ over time for the SOS and FOS schemes for this spherical mode of vibration. The plot on the left shows the behavior over the time interval $[0,5]$ while the plot on the right shows the behavior for the longer interval $[0,50]$. We observe that there is a large variation in the maximum errors over time, but on average the errors for the FOS scheme are smaller than those for the SOS scheme and for both schemes the errors grow approximately linearly in time due to the accumulation of truncation errors. Linear least-squares fits to the data illustrate this behavior and show that the errors grow as .0022t and .0016t for the SOS and FOS schemes, respectively.
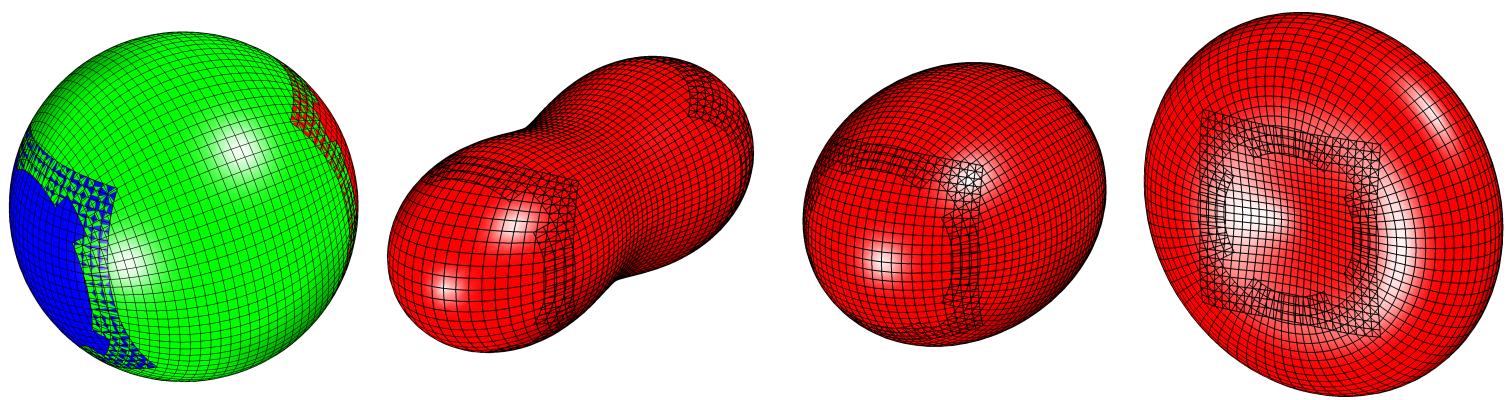

Figure 24: Vibrational mode of an elastic sphere. Left: overlapping grid for a solid sphere consisting of two orthographic patches, a spherical polar shell and an interior Cartesian grid (not visible). Middle to right: the deformed sphere at times $t=0, t=0.8$ and $t=1.2$ for the case $\rho=\lambda=\mu=1$ and $R=1$. The displacement is scaled by a factor of 0.08 for illustrative purposes.

\subsection{Diffraction of a p-wave "shock" by a circular cavity}

To illustrate the use of adaptive mesh refinement we consider the diffraction of a p-wave "shock" by a circular cavity. The domain for this problem is taken to be the two-dimensional region interior to the square $[-3,3]^{2}$ and exterior to the circle of radius $R=0.5$. The initial conditions are taken from the exact traveling-wave solution for a planar p-wave with a piecewise constant velocity profile. The displacement and velocity for this solution are given by

$$
\mathbf{u}(\xi)=\left\{\begin{array}{ll}
-\xi\left(v_{0} / c_{p}\right) \boldsymbol{\kappa} & \text { for } \xi<0, \\
0 & \text { for } \xi>0,
\end{array} \quad \mathbf{v}(\xi)= \begin{cases}v_{0} \boldsymbol{\kappa} & \text { for } \xi<0, \\
0 & \text { for } \xi>0,\end{cases}\right.
$$




\begin{tabular}{|c|c|c|c|c|c|c|c|c|c|}
\hline & & \multicolumn{2}{|c|}{ SOS } & \multicolumn{6}{|c|}{ FOS } \\
\hline Grid $\mathcal{G}^{(j)}$ & $h_{j}$ & $e_{u}^{(j)}$ & $r$ & $e_{u}^{(j)}$ & $r$ & $e_{v}^{(j)}$ & $r$ & $e_{\sigma}^{(j)}$ & $r$ \\
\hline $\mathcal{G}_{\mathrm{ss}}^{(1)}$ & $1 / 10$ & $1.3 \times 10^{-1}$ & & $5.1 \times 10^{-2}$ & & $1.2 \times 10^{-1}$ & & $2.6 \times 10^{-1}$ & \\
\hline $\mathcal{G}_{\mathrm{Ss}}^{(2)}$ & $1 / 20$ & $4.0 \times 10^{-2}$ & 3.2 & $1.2 \times 10^{-2}$ & 4.2 & $3.0 \times 10^{-2}$ & 4.0 & $5.1 \times 10^{-2}$ & 5.1 \\
\hline $\mathcal{G}_{\mathrm{ss}}^{(4)}$ & $1 / 40$ & $1.0 \times 10^{-2}$ & 4.0 & $2.4 \times 10^{-3}$ & 5.1 & $7.1 \times 10^{-3}$ & 4.2 & $8.6 \times 10^{-3}$ & 6.0 \\
\hline $\mathcal{G}_{\mathrm{SS}}^{(8)}$ & $1 / 80$ & $2.4 \times 10^{-3}$ & 4.1 & $5.2 \times 10^{-4}$ & 4.6 & $1.7 \times 10^{-3}$ & 4.1 & $2.0 \times 10^{-3}$ & 4.3 \\
\hline \multicolumn{2}{|c|}{ rate } & 1.93 & & 2.22 & & 2.03 & & 2.37 & \\
\hline
\end{tabular}

Figure 25: Maximum errors and estimated convergence rates for numerical solutions of a vibrational mode of a solid sphere using the SOS and FOS schemes.
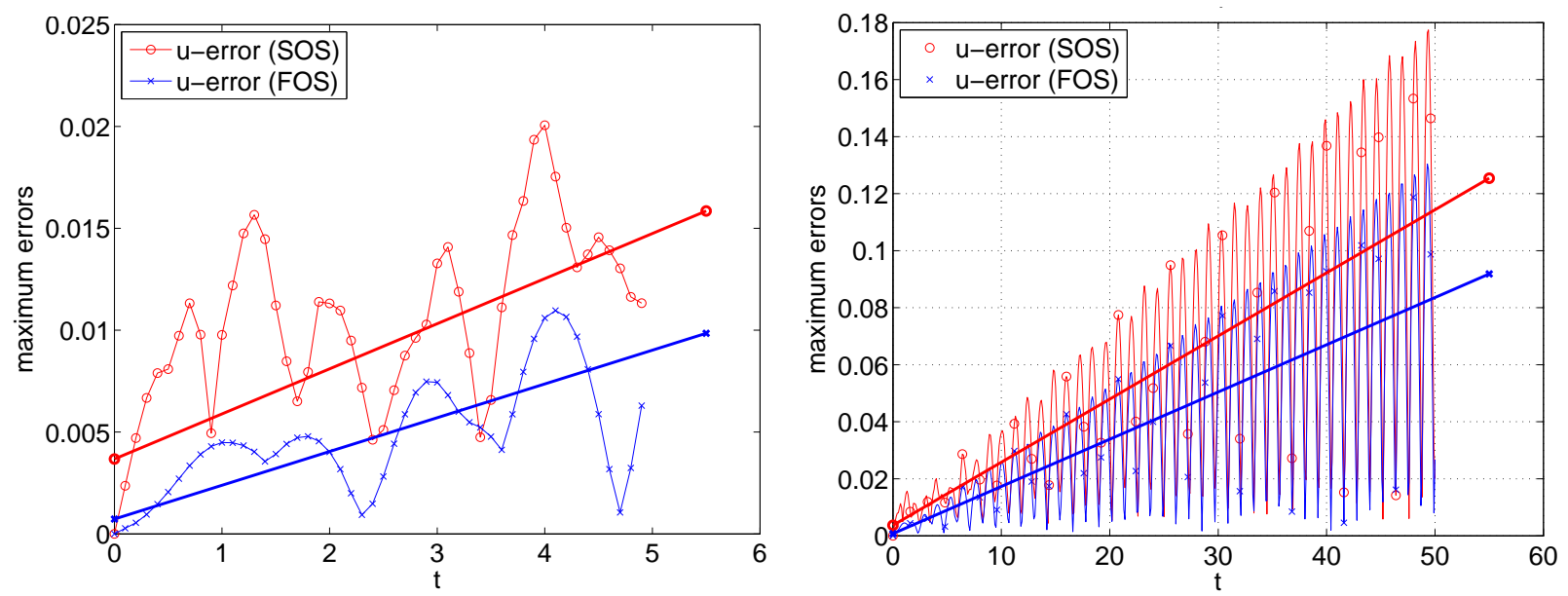

Figure 26: Vibrational mode of an elastic sphere: maximum errors in $\mathbf{u}$ over time for the SOS and FOS schemes on grid $\mathcal{G}_{\mathrm{ss}}^{(4)}$. The total number of time steps to integrate to $t=50$ was approximately 10,000 for the SOS scheme and 15,000 for the FOS scheme.

where

$$
\xi=\kappa \cdot\left(\mathbf{x}-\mathbf{x}_{0}\right)-c_{p} t, \quad c_{p}=\sqrt{(\lambda+2 \mu) / \rho} .
$$

Here, $\boldsymbol{\kappa}$ defines the direction of propagation of the planar wave and $\mathbf{x}_{0}$ defines its position at $t=0$. For the computations presented, we take $\boldsymbol{\kappa}=(1,0), v_{0}=c_{p}$ and $\mathbf{x}_{0}=(-1.25,0)$, and we assume that $\rho=\lambda=\mu=1$. The boundary conditions on the bottom and top sides of the square are slip-wall conditions, and exact data from the planar p-wave solution is used as Dirichlet conditions on the left and right sides of the square. A homogeneous traction condition is applied on the boundary of the circular cavity.

Calculations are performed using the SOS and FOS schemes with one level of AMR grids using a refinement factor of $n_{r}=2$ or 4 . The base-level composite grid for the two-dimensional domain is composed of a rectangular Cartesian grid defined previously in (75) and an annular grid defined in (74). This circle-in-asquare grid is defined by

$$
\mathcal{G}_{C S}^{(j)}=\mathcal{R}\left([-3,3]^{2}, N_{x}(j), N_{x}(j)\right) \cup \mathcal{C}\left(\left[R, R+7 h_{j}\right], N_{\theta}(j), N_{r}\right),
$$

where $h_{j}=1 /(10 j)$ gives the approximate grid spacing, and the number of grid cells in the various coordinate directions are given by

$$
N_{x}(j)=\left\lfloor 6 / h_{j}+1.5\right\rfloor, \quad N_{\theta}(j)=\left\lfloor 2 \pi\left(R+3.5 h_{j}\right) / h_{j}+1.5\right\rfloor, \quad N_{r}=7 .
$$

We note that the composite grid uses a boundary-fitted annular grid with a fixed number of grid cells in the radial direction which is similar to the "narrow" grid, $\mathcal{G}_{D n}^{(j)}$, used in Section 6.1.

Figure 27 shows the elastic response of the planar p-wave as it is diffracted by the circular cavity. The numerical solution is computed using the FOS scheme with the base-level composite grid given by $\mathcal{G}_{C S}^{(8)}$ and the addition of one refinement level with $n_{r}=4$. The plots show shaded contours of the magnitude of velocity at times $t=0,1.0$ and 1.6. When the p-wave meets the cavity, the boundary of the cavity is deformed and a reflected wave is created. The reflected wave consists of both pressure and shear waves which travel at different velocities as seen clearly in the plot at $t=1.0$. The cavity continues to deform as the diffracted 
waves travels around it, and ultimately the waves collide near the back of the cavity as seen in the plot at $t=1.6$. The behavior of the refinement grids are shown in the figure, and these grids are well-positioned during the calculation to increase the grid resolution of the various waves as they move in time throughout the domain.
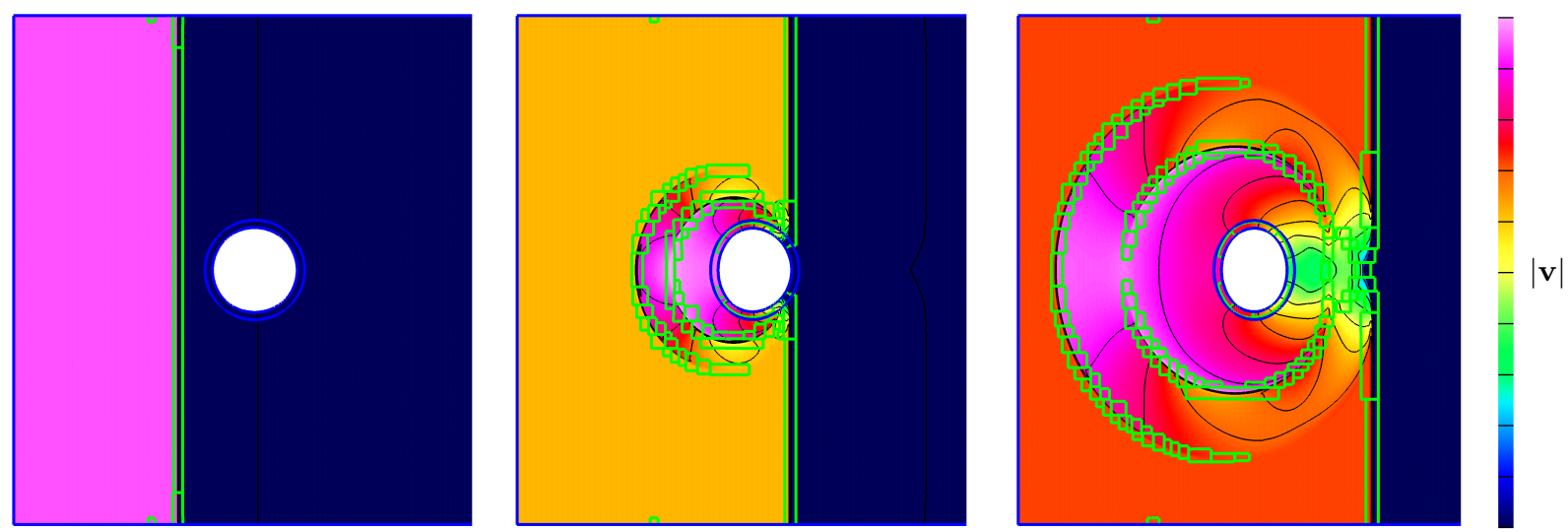

Figure 27: Diffraction of a p-wave shock by a circular cavity. Numerical solution computed with AMR using one refinement level with $n_{r}=4$ and the FOS scheme. Shaded contours of the magnitude of velocity at times (from left to right) $t=0$ (contour bounds $[0,1.82]), t=1.0([0,2.79])$ and $t=1.6([0,2.41])$. The boundaries of the base-level component grids are shown in blue and the boundaries of the refinement grids are shown in green. The deformed grid is shown using a scaling of the displacement by a factor of 0.075 .

Figure 28 compares the results for the SOS and FOS schemes for an AMR computation. For this comparison, we use the base grid $\mathcal{G}^{(8)}$ together with one level of $n_{r}=4$ refinement. The magnitudes of the displacement and velocity at time $t=1.6$ are shown. From the figures it can be seen that the results from both schemes are generally in good agreement. The FOS results are less noisy than the SOS results, particularly in $|\mathbf{v}|$, which might be expected from the FOS approach as it is an upwinding scheme. Note, however, that the velocity is directly computed by FOS while for the SOS scheme it is computed in a postprocessing step by a finite difference approximation in time, $\mathbf{v}_{\mathbf{i}}^{n}=\left(\mathbf{u}_{\mathbf{i}}^{n}-\mathbf{u}_{\mathbf{i}}^{n-1}\right) / \Delta t$, and this may contribute to some of the noise.

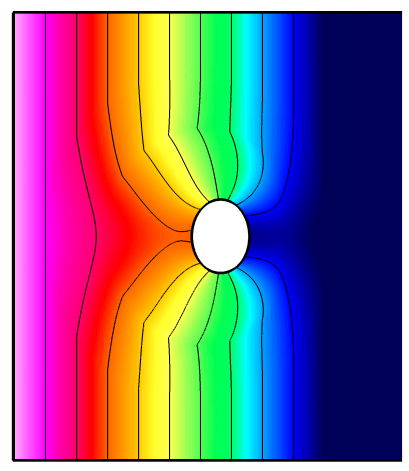

SOS with AMR

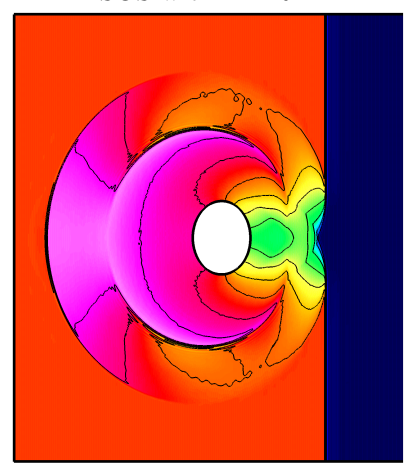

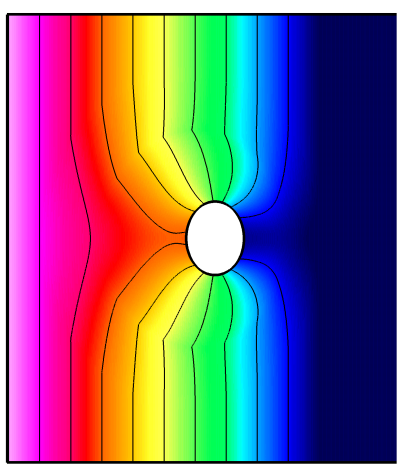

FOS with AMR

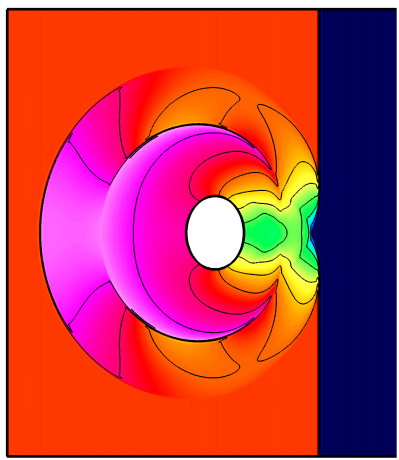

4.5

$|\mathbf{u}|$

0.0

2.4

0.0

Figure 28: Diffraction of a traveling p-wave "shock" by a circular cavity at time $t=1.6$ showing the norms of the displacement and velocity for the SOS scheme (left column) and FOS scheme (right column). Results are for the base grid $\mathcal{G}^{(8)}$ using one refinement level with $n_{r}=4$. The contours are plotted on the deformed grid, scaling the displacement by a factor of 0.075 . 
The accuracy of the AMR computations for this example can be made more quantitative. Given a sequence of three grids of increasing resolution, a posteriori estimates of the errors and convergence rates can be computed using the procedure described in [10]. These self-convergence estimates assume that the numerical results are converging to some limiting solution. A posteriori estimates computed in this way are given in Figure 29 for three grids of increasing resolution. The coarse grid computation used the base grid $\mathcal{G}_{C S}^{(2)}$ together with one refinement level of factor 2. The medium resolution computation used the base grid $\mathcal{G}_{C S}^{(2)}$ together with one refinement level of factor 4 , while the finest resolution used the grid $\mathcal{G}_{C S}^{(64)}$ with no AMR. The parameters in the AMR error estimate (10) were chosen as $c_{1}=0, c_{2}=1$ and $s_{k}=1$. The error tolerances for the SOS scheme were taken as $4 \times 10^{-3}$ and $1 \times 10^{-3}$ for the coarse and medium resolutions respectively. The corresponding error tolerances for the FOS scheme were $10^{-2}$ and $2.5 \times 10^{-3}$ respectively.

The figure provides estimates of the $L_{1}$-norm errors. Since the exact solution for the displacement has discontinuous first derivatives, one cannot expect second-order accurate convergence. We expect that the $L_{1}$-norm error in the displacement would converge at rate of 1 in the limit of small $h$, while the errors in velocity and stress would converge at a rate of $2 / 3$, see [34]. The results in the figure indicate that the computed convergence rates are close to the expected rates.

\begin{tabular}{|c|c|c|c|c|c|c|c|c|c|c|c|}
\hline \multicolumn{2}{|c|}{} & \multicolumn{3}{c|}{ SOS } & \multicolumn{5}{c|}{ FOS } \\
\hline Grid & levels & $n_{r}$ & $h_{j}$ & $e_{u}^{(j)}$ & $r$ & $e_{u}^{(j)}$ & $r$ & $e_{v}^{(j)}$ & $r$ & $e_{\sigma}^{(j)}$ & $r$ \\
\hline $\mathcal{G}_{C S}^{(2)}$ & 2 & 2 & $1 / 40$ & $8.9 \times 10^{-4}$ & & $2.1 \times 10^{-3}$ & & $1.0 \times 10^{-2}$ & & $1.2 \times 10^{-2}$ & \\
\hline $\mathcal{G}_{C S}^{(2)}$ & 2 & 4 & $1 / 80$ & $4.7 \times 10^{-4}$ & 1.9 & $1.0 \times 10^{-3}$ & 2.0 & $6.4 \times 10^{-3}$ & 1.6 & $7.2 \times 10^{-3}$ & 1.6 \\
\hline $\mathcal{G}_{C S}^{(64)}$ & - & - & $1 / 640$ & $7.1 \times 10^{-5}$ & 6.7 & $1.2 \times 10^{-4}$ & 8.5 & $1.5 \times 10^{-3}$ & 4.3 & $1.7 \times 10^{-3}$ & 4.3 \\
\hline \multicolumn{3}{|c|}{ rate } & 0.91 & & 1.03 & & 0.70 & & 0.71 & \\
\hline
\end{tabular}

Figure 29: A posteriori estimated errors $\left(L_{1}\right.$-norm) and convergence rates at $t=1.0$ for diffraction of a p-wave "shock" by a circular cavity using AMR. Note that the finest grid is a factor 8 times finer than the previous resolution.

\subsection{Three-dimensional circular plate with holes}

As a final example, we consider an elastic disturbance propagating in a three-dimensional circular plate with holes, see Figure 30. Without holes, the circular plate would occupy the domain $\Omega_{p}=\left\{\left(x_{1}, x_{2}, x_{3}\right) \mid x_{1}^{2}+\right.$ $\left.x_{2}^{2} \leq R, 0 \leq x_{3} \leq W\right\}$, where the outer radius of the plate is taken to be $R=4$ and its thickness is taken to be $W=0.25$. Twenty-four small holes of radius 0.3 are located on equally spaced angles, $\theta_{k}=(15 k)^{\circ}$, $k=0,1, \ldots, 23$, with centers on a radius of $r=3.4$. Twelve larger holes of radius 0.4 are equally spaced on angles $\theta_{k}=(30 k+15)^{\circ}, k=0,1, \ldots, 11$, with centers on a radius of $r=2.25$. Let $\mathcal{G}_{P}^{(j)}$ denote the overlapping grid for this domain, where the grid spacing is approximately equal to $h_{j}=1 /(10 j)$. The overlapping grid, shown in the left plot of Figure 30, is composed of 38 component grids. A Cartesian box grid defined in (77) is used to cover the bulk of the interior of the domain, while a cylindrical boundary-fitted grid is used to represent the outer boundary of the plate. (The cylindrical grid is a straightforward extension of the annulus grid defined in (74).) The remaining 36 component grids are smaller cylindrical grids that define the boundaries of the holes in the plate. We note that for this composite grid, the grid spacings on the component grids are well matched so that for grid $\mathcal{G}_{P}^{(16)}$, for example, the global time step is only smaller by a factor of 0.97 as compared to the maximum allowable time step for any component grid.

It is assumed that the plate is at rest initially so that the displacement and velocity are both set to zero at $t=0$. Traction boundary conditions are applied on all surfaces of the plate. The applied stress given by $\mathbf{g}_{t}(\mathbf{x}, t)$ in (5) is taken to be zero for all surfaces except for the top surface at $x_{3}=W$ where a nonzero time-dependent stress is applied which sets the plate in motion. The applied stress is taken to be

$$
\mathbf{g}_{t}\left(x_{1}, x_{2}, W, t\right)=-g(t) \exp \left[-20\left(x_{1}^{2}+x_{2}^{2}\right)\right] \hat{\mathbf{e}}_{3},
$$

where $\hat{\mathbf{e}}_{3}$ is the unit vector is the $x_{3}$ direction, and the amplitude of the applied stress is given by

$$
g(t)= \begin{cases}A t^{p}(1-t)^{p}(1 / 2-t), & 0 \leq t \leq 1, \\ 0, & \text { otherwise. }\end{cases}
$$

Here, $A$ determines the overall strength of the applied stress and $p$ is a positive integer chosen to be 3 so that the forcing turns on and off smoothly. Note that $g(t)$ is an odd function about $t=1 / 2$ so that the integral from $t=0$ to 1 is zero. Also, we take $A=2048$ so that the integral on the half interval $t=0$ to $1 / 2$ 
is one. The applied stress given by (81) is concentrated near the center of the plate, and acts to first push down on the plate and then to pull it back up before it turns off at $t=1$. The elastic response of the plate is complex due to the presence of the holes, and numerical solutions are obtained using the SOS and FOS schemes for the case $\rho=\lambda=\mu=1$. The larger computations presented in this section were computed in parallel on up to 128 processors (see Section 6.8 for some performance statistics of the two schemes).

Figure 30 shows the evolution of the surface displacement of the plate at times $t=2,2.5, \ldots, 4$ computed using the SOS scheme on grid the $\mathcal{G}_{P}^{(8)}$. We observe that the disturbance generally propagates outward from the center of the plate towards its perimeter. As the disturbance meets the holes, the leading edge of the wave diffracts around the holes and reflects back towards the center of the plate. This interaction occurs first with the inner ring of larger holes, and then later with the outer ring of smaller holes. Each interaction generates more waves, and by the final time shown in the figure, the surface displacement is a complex distribution of elastic waves.
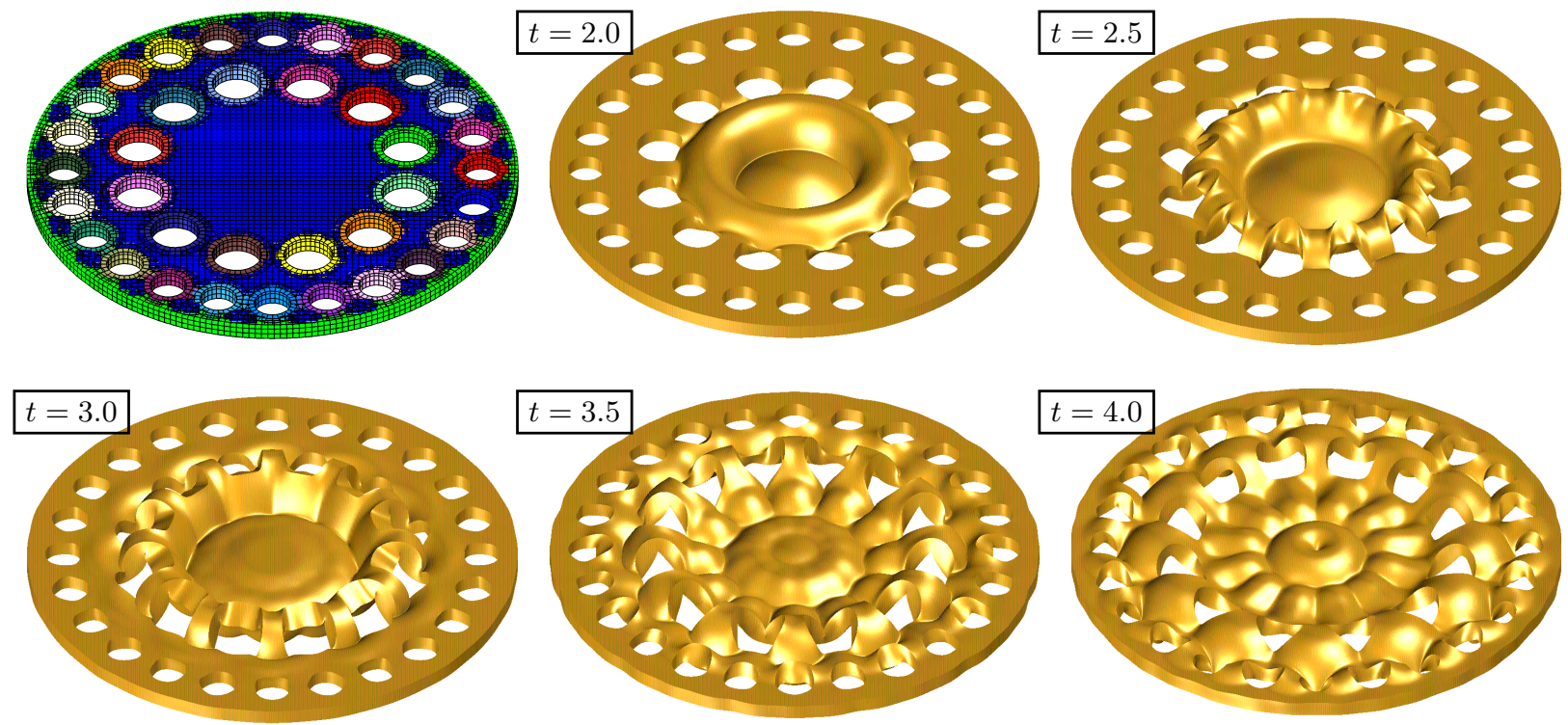

Figure 30: Elastic waves in a three-dimensional circular plate with holes, composite grid (coarsened) and surface displacement at selected times. The deformed surface is shown with the displacement scaled by a factor of 3 .

The accuracy of these complex three-dimensional simulations can be made more quantitative (following the procedure described previously in Section 6.6) by computing a posteriori estimates of the errors and convergence rates using solutions from a sequence of three grids of increasing resolution [10]. The a posteriori estimates computed in this way are given in Figure 31 for grids $\mathcal{G}_{P}^{(j)}$ with $j=4,8,16$. The finest grid, $\mathcal{G}_{P}^{(16)}$, has about 42 million active grid points. The estimated convergence rates for the maximum errors are close to the expected value of two for both schemes.

\begin{tabular}{|c|c|c|c|c|c|c|c|c|c|}
\hline \multicolumn{2}{|c|}{} & \multicolumn{6}{|c|}{ SOS } & \multicolumn{5}{c|}{ FOS } \\
\hline Grid $\mathcal{G}^{(j)}$ & $h_{j}$ & $e_{u}^{(j)}$ & $r$ & $e_{u}^{(j)}$ & $r$ & $e_{v}^{(j)}$ & $r$ & $e_{\sigma}^{(j)}$ & $r$ \\
\hline $\mathcal{G}_{P}^{(4)}$ & $1 / 40$ & $1.8 \times 10^{-2}$ & & $9.5 \times 10^{-3}$ & & $1.0 \times 10^{-1}$ & & $1.3 \times 10^{-1}$ & \\
\hline $\mathcal{G}_{P}^{(8)}$ & $1 / 80$ & $4.5 \times 10^{-3}$ & 3.9 & $2.2 \times 10^{-3}$ & 4.2 & $2.8 \times 10^{-2}$ & 3.6 & $3.2 \times 10^{-2}$ & 3.9 \\
\hline $\mathcal{G}_{P}^{(16)}$ & $1 / 160$ & $1.2 \times 10^{-3}$ & 3.9 & $5.3 \times 10^{-4}$ & 4.2 & $7.7 \times 10^{-3}$ & 3.6 & $8.2 \times 10^{-3}$ & 3.9 \\
\hline \multicolumn{2}{|c|}{ rate } & 2.0 & & 2.1 & & 1.9 & & 2.0 & \\
\hline
\end{tabular}

Figure 31: A posteriori estimated errors (max-norm) and convergence rates for computing elastic waves in a three-dimensional circular plate with holes at $t=3.0$.

\subsection{Performance of the SOS and FOS schemes}

We close the discussion of the results with a comparative study of the performance of the SOS and FOS schemes. This is done using a calculation of a vibrational mode of a solid sphere as a representative problem (see Section 6.5). For this three-dimensional calculation, the SOS scheme advances 3 components of displacement per grid point at each time step. The FOS scheme, on the other hand, advances 15 solution components per grid point at each time step, i.e. 3 components for displacement, 3 for velocity and 9 for 
stress. There would be some computational saving to be gained in our implementation if the symmetry of the stress tensor were exploited, but we would still expect a scheme based on the second-order form of the equations to be faster per time step than a scheme based on the first-order form (as is confirmed by the CPU timings below). In our FOS scheme, we advance all components of stress for simplicity, but there is some optimization employed in the calculation of the flux for the symmetric components of the stress. The FOS scheme also retains the displacement in addition to the velocity and stress. Updating the displacement using the velocity in (32) requires relatively little computational effort, and thus the main effect of keeping the displacement is to increase the storage requirements from 12 to 15 components (for three space dimensions). In addition, both schemes use optimized computational kernels for the case of Cartesian component grids. In general, we have made a modest attempt to optimize the codes for the SOS and FOS schemes, so that the timings below provide a reasonable indication of the relative computational cost of the two schemes.

Figure 32 gives CPU timings for the computation of a vibrational mode in a sphere using the overlapping grid $\mathcal{G}_{\mathrm{ss}}^{(20)}$ with approximately 34 million grid points. This grid has four component grids with the majority of the grid points (32 million) belonging to the Cartesian box grid in the interior of the domain. For this composite grid there are 1.1 million overlapping-grid interpolation points. The computation for each scheme was run in parallel on 16 processors (4 nodes with 4 processors per node) using 2.4Ghz AMD Opteron processors with $16 \mathrm{~Gb}$ per node. The CPU times reported in the figure for the SOS and FOS schemes are the averaged values over the 16 processors. The row labeled "advance" in the figure denotes the time spent advancing the solutions at interior grid points (performed using optimized Fortran routines) and does not include communication costs. The row labeled "interpolation" includes the cost for overlappinggrid interpolation as well as the cost for updating the two layers of parallel ghost values. There is a cost associated with applying the boundary conditions at each step and a cost to apply the filter for the SOS scheme. Both of these are listed in the figure. All other costs are listed as "other."

\begin{tabular}{|r|r|r|r||r|r|r|}
\cline { 2 - 7 } & \multicolumn{7}{c|}{ Vibration of a Sphere (3D) } \\
\cline { 2 - 8 } & \multicolumn{3}{|c|}{ SOS } & \multicolumn{3}{|c|}{ FOS } \\
\cline { 2 - 8 } advance & total (s) & s/step & $\%$ & total (s) & s/step & $\%$ \\
\cline { 2 - 8 } boundary conditions & 90 & 1.3 & 45 & 2005 & 16.9 & 67. \\
interpolation & 15 & .22 & 7 & 51 & .42 & 1.7 \\
filter & 41 & .60 & 20 & 876 & 7.4 & 29. \\
other & 40 & .58 & 20 & - & - & - \\
total & 5 & .10 & 8 & 30 & .18 & 2.3 \\
\cline { 2 - 8 } & 190 & 2.8 & 100 & 2962 & 24.9 & 100 \\
\hline
\end{tabular}

Figure 32: Performance of the SOS and FOS schemes for the calculation of a vibrational mode in a solid sphere using the composite grid $\mathcal{G}_{\mathrm{ss}}^{(20)}$ with approximately 34 million grid points. CPU time (in seconds) for various parts of the SOS and FOS codes, and their percentages of the total CPU time per step. The computation was run in parallel on 16 processors.

The timings for the SOS scheme show that approximately half of the time per step ( $45 \%$ of the total time) is taken to advance the solution following the discretization described in Section 4.1, while the application of the sixth-order filter is relatively expensive ( $20 \%$ of the total time). This is not surprising given that the filter is applied in two stages and requires a separate parallel ghost boundary update for each stage. In addition, the majority of grid points belong to a Cartesian grid where the discretization of the governing equations requires relatively few operations. The optimized computational kernel exploits this so that this part of the SOS code is very efficient. The interpolation and parallel ghost-point update is also relatively expensive at $20 \%$. This is due, in part, to the parallel load balancer which does not currently take the work required for interpolation into account and as a result the computation is not perfectly balanced. We also note that the Cartesian grid implementation for the SOS scheme in three dimensions is almost 10 times faster per grid point than the version for curvilinear grids. Thus the code runs significantly faster when a majority of grid points reside on Cartesian grids.

For the FOS scheme, approximately $67 \%$ of the time is spent advancing the solution following the discretization in Section 4.2. This is to be expected since the FOS scheme requires significantly more operations per time step than that for the SOS scheme. The next highest cost per step is due to interpolation and the parallel ghost-point update. The cost for this is also more than that for the SOS scheme due to the larger number of solution components involved. Overall, the results in Figure 32 indicate that the FOS scheme is about 24.9/2.8 = 8.9 times slower per time step than the SOS scheme for this calculation. In addition, the time step, $\Delta t$, for the FOS scheme (33), is smaller than that for the SOS scheme (23), by a factor of approximately $1 / \sqrt{3} \approx 0.6$. This results in the SOS calculation being about 15.6 times faster than the FOS one. Also, the SOS scheme used about one-third of the memory of the FOS scheme (0.6 Gb of memory per 
processor compared to $1.8 \mathrm{~Gb}$ ).

Although the FOS scheme is more expensive in terms of computational time and memory, its solution is often more accurate than the solution obtained using the SOS scheme for the same composite grid. A solution computed using the FOS scheme on a coarser grid (which requires less computational cost) may still be more accurate than the solution using the SOS scheme on a finer grid. For example, the time required for a time-dependent simulation in three space dimensions decreases by a factor of 16 , roughly, when the mesh spacing $h$ increases by a factor of 2 . The error, on the other hand, only increases by a factor of 4 for the same increase in the mesh spacing. Since the FOS scheme is roughly 16 times slower than the SOS scheme (as our current implementations suggest), a FOS calculation of a three-dimensional problem on a grid with mesh spacing $2 h$ would cost about the same as an SOS calculation of the same problem on a grid with mesh spacing $h$. If the error in the solution of the FOS scheme is more than 4 times smaller than that given by the SOS scheme on the same grid, then the FOS scheme may be a better choice (using a coarser grid). The results in the previous sections suggest that this can often be the case.

\section{Accuracy and points per wavelength}

One measure of the performance of a numerical scheme for wave propagation problems is given by the number of grid points per wavelength needed to obtain a given accuracy over a given time interval [35]. An estimate of this measure can be derived by determining the relative error in approximating a periodic plane wave solution of the form $\exp (i k(x-c t))$. For a $p^{\text {th }}$-order accurate spatial approximation to the first order wave equation, $u_{t}+c u_{x}=0$, the number of points per wavelength $\mathcal{N}_{\lambda}$ to achieve a relative error $\epsilon$ over a time interval of $T$ periods is given by

$$
\mathcal{N}_{\lambda} \approx K_{p}\left(\frac{T}{\epsilon}\right)^{1 / p}
$$

where the coefficient $K_{p}$ is a measure of the truncation error [33]. The advantage of using high-order accurate schemes (i.e. a large value of $p$ ) is clear from (82), especially for long time integrations or small error tolerances. Following the approach in [33], we can estimate $K_{p}$ for the SOS and FOS schemes by considering the fully discrete second-order accurate approximation to the one-dimensional second-order wave equation (SOWE) in (51) (with $a_{d}=0$ ), and the fully discrete version of the Godunov scheme in (69) for the first-order wave equation (FOWE). This gives

$$
K_{p}^{\mathrm{SOWE}}(\sigma) \approx \sqrt{1-\sigma^{2}} \pi\left(\frac{\pi}{3}\right)^{1 / 2}, \quad K_{p}^{\mathrm{FOWE}}(\sigma) \approx \sqrt{2|(1-\sigma)(1-2 \sigma)|} \pi\left(\frac{\pi}{3}\right)^{1 / 2} .
$$

where $\sigma=c \Delta t / h$ is the (one-dimensional) CFL parameter. Note that both one-dimensional schemes are exact for $\sigma=1$, while the Godunov scheme is third-order accurate for $\sigma=1 / 2$.

Actual computations of a plane wave solution using the SOS and FOS schemes on a two-dimensional periodic square with a (two-dimensional) CFL number of 0.9 , provide estimates for the relative errors in $\mathbf{u}$ to the elastodynamic equations. This provides values for $\epsilon, T$, and $\mathcal{N}_{\lambda}$ that, when used in (82), allows one to estimate $K_{P}$ as

$$
K_{p}^{\mathrm{SOS}} \approx 2.5, \quad K_{p}^{\mathrm{FOS}} \approx 1.1 .
$$

We note that the values of $K_{p}$ in (84) depend on the CFL number and the direction of propagation of the wave; we have chosen the wave to propagate in the $x$-direction. The approximations in (84) are in very good agreement with the results of the one-dimensional theory (83) when we choose $\sigma=.634$ for the SOWE, and $\sigma=.447$ for the FOWE (to match the value of $\Delta t$ used in the two-dimensional computations), i.e.

$$
K_{p}^{\mathrm{SOWE}}(\sigma=.634) \approx 2.5, \quad K_{p}^{\mathrm{FOWE}}(\sigma=.447) \approx 1.1 .
$$

The approximations in (84), together with (82), can be used to estimate the expected errors in our numerical simulations. For the computations of a vibrating sphere in Section 6.5, for example, we estimate the period of oscillation to be $P \approx 2.4$, the wavelength to be $2 R$ and $\mathcal{N}_{\lambda} \approx 20 j$ for the grid $\mathcal{G}_{\mathrm{ss}}^{(j)}$. This gives the error estimates

$$
\begin{aligned}
\epsilon^{\mathrm{SOS}} & \approx(2.5)^{2} T \mathcal{N}_{\lambda}^{-2} \approx 1.6 \times 10^{-2} T j^{-2}, \\
\epsilon^{\mathrm{FOS}} & \approx(1.1)^{2} T \mathcal{N}_{\lambda}^{-2} \approx 3.0 \times 10^{-3} T j^{-2} .
\end{aligned}
$$


The computed errors at $t=0.5(T=t / P \approx .21)$ are given in Figure 25 . For $j=8$ we obtain $\epsilon^{\mathrm{SOS}} \approx 5 \times 10^{-5}$ (compared to the actual value $\left.8 \times 10^{-4}\right)$ and $\epsilon^{\mathrm{FOS}} \approx 1 \times 10^{-5}\left(\right.$ compared to the actual value of $\left.4.7 \times 10^{-5}\right)$. Given the approximations involved (e.g. no consideration of boundary conditions) and the fact that the errors vary significantly with time (as shown in Figure 26) the error estimates (86)-(87) seem to provide a reasonable approximation to the actual values. The analysis also supports the observation that the FOS scheme is more accurate than the SOS scheme, in general.

\section{Conclusions}

We have conducted the first careful examination of the use of overlapping grids and AMR for the solution of the equations of linear elasticity. We have compared two different approaches, the SOS scheme and the FOS scheme, in terms of accuracy, stability and computational performance. The accuracy and stability of the schemes was examined for a wide range of test problems in both two and three dimensions involving different constitute parameters, domain geometries and overlapping-grid configurations, and types of boundary conditions. These problems also provide valuable benchmark solutions for time dependent elasticity. The method of analytic solutions was used to construct exact solutions for some of the test problems, while exact solutions were known for two other problems involving a deforming annulus and deforming solid sphere. These solutions were used to show that both schemes are second-order accurate (for smooth solutions). A posteriori error estimates were used to study the accuracy of the schemes for two problems in which exact solutions are not known. These problems involved the elastic response of a three-dimensional circular plate with holes, and the diffraction of a p-wave "shock" by a circular cavity. The solution of the plate problem is smooth and estimates of the error confirmed second-order accuracy of the numerical solutions. The solution of the diffraction problem, on the other hand, is not smooth, and error estimates in the 1-norm were obtained and found to be in agreement with the expected convergence rates. As a general rule, it was found that the numerical solution given by the FOS scheme was more accurate than that given by the SOS scheme, although our study of the performance of the schemes for a representative test problem showed that the FOS was more expensive computationally. However, depending on the problem, the computational effort required to achieve a given level of accuracy may be smaller for the FOS scheme.

The numerical schemes run significantly faster on Cartesian grids. Thus, for good computational efficiency one should construct the overlapping grid to maximize the number of Cartesian grid points. If boundary fitted curvilinear grids are constructed with a fixed number of points in the normal direction, then as the grid is refined the ratio of curvilinear grid points to Cartesian grid points will approach zero and the overall cost of the scheme will approach that for Cartesian grids.

We have provided the first demonstration of the use of overlapping grids and block-structured AMR for elasticity problems as a means to locally increase the grid resolution as the solution evolves in time. The AMR technique was illustrated using the problem of the diffraction of a p-wave "shock" by a circular cavity. In addition, some of the numerical tests, including the test of performance, were computed in parallel on a distributed-memory computer.

An analysis of the scalar wave equation in second-order and first-order form on a one-dimensional domain showed the new result that non-dissipative schemes (that are stable on a single grid) may have unstable modes on overlapping grids with growth rates proportional to the inverse of the mesh spacing. These unstable modes are generated by waves that become trapped in the region between a grid overlap and a nearby boundary. This result, which dictates how the dissipation must scale as the mesh is refined, is important when solving any type of wave propagation problem on overlapping grids including Maxwell's equations, for example. In particular, this instability appears when solving the elastodynamic equations. A particularly difficult situation occurs with traction boundary conditions and the SOS scheme when the ratio $\lambda / \mu$ is large. In this case, our numerical tests showed that the SOS scheme can be stabilized by the addition of an appropriate artificial dissipation and high-order filter. A sixth-order filter together with a small fourth-order dissipation was found to be an effective choice. The FOS scheme has inherent high-order dissipation that scales in the correct way as the mesh is refined and numerical tests indicate that this is sufficient to suppress this type of instability.

In future work we plan to evaluate high-order accurate approximations and, in addition, consider elastodynamic problems with material interfaces, variable material coefficients, and large (nonlinear) deformations. The numerical approach developed here (and its extensions) will also be used in the context of fluid-structure interaction problems. 


\section{Appendix A. Boundary conditions}

In this appendix we describe our implementation of the displacement (4), traction (5) and slip-wall (6) boundary conditions for the discretizations of the second-order and first-order systems described in Sections 4.1 and 4.2, respectively. For the second-order system, care must be taken in approximating the traction boundary condition to avoid instabilities when the ratio $\lambda / \mu$ becomes large. In the case of the first-order system, the typical approach at boundaries would only use approximations involving components of the velocities and stresses [36]. We, however, take a different approach and also couple the components of the displacement into the boundary conditions, as described in Appendix A.2.

At a time $t_{n}-\Delta t$, say, it is assumed that the discrete solution is available at all interior grid points, as well as grid points on the boundary and in the necessary ghost points (depending on the width of the stencil). The algorithms outlined previously in Sections 4.1 and 4.2 are used to advance the solution at all grid points in the interior and on the boundary, and boundary conditions are then applied at the current time $t_{n}$. This involves setting values in the ghost points and, possibly, overwriting values on the boundary depending on the boundary condition type and the particular components of the solution involved. Further details are discussed in the subsections below for the discretizations of the second-order and first-order system.

Appendix A.1. Boundary conditions for the second-order system

The displacement boundary condition in (4) is straightforward to implement in the SOS scheme by setting

$$
\mathbf{u}_{\mathbf{i}}^{n}=\mathbf{g}_{d}\left(\mathbf{x}_{\mathbf{i}}, t_{n}\right),
$$

for all points $\mathbf{i}$ on the boundary face. Discrete values at ghost points, needed to apply the filter in (21) for example, are obtained by extrapolation. For the boundary face $r_{1}=0$ with $i_{1}=0$, a third-order extrapolation of the discrete value at the ghost point $\mathbf{i}=\left(-1, i_{2}, i_{3}\right)$ is given by

$$
\mathbf{u}_{-1, \mathbf{j}}^{n}=\mathcal{E}_{+1}^{(3)} \mathbf{u}_{0, \mathbf{j}}^{n}=3 \mathbf{u}_{0, \mathbf{j}}^{n}-3 \mathbf{u}_{1, \mathbf{j}}^{n}+\mathbf{u}_{2, \mathbf{j}}^{n},
$$

where $\mathbf{j}=\left(i_{2}, i_{3}\right)$ denotes the indices in the directions tangential to the boundary. We use this third-order extrapolation to obtain discrete values at ghost points for the displacement boundary condition.

Application of the traction boundary condition in (5) for the SOS scheme requires more care. Generally, we use the traction boundary condition to assign values of the solution on the first ghost line, $\mathbf{u}_{-1, \mathbf{j}}^{n}$ for example, while values of the solution on the boundary are obtained using the interior equations (as mentioned above). The traction boundary condition defines Neumann-like conditions on $\mathbf{u}$. For example, in the twodimensional Cartesian case for a boundary on $x_{1}=0$, the conditions are

$$
\frac{\partial u_{1}}{\partial x_{1}}=-\frac{\lambda}{\lambda+2 \mu} \frac{\partial u_{2}}{\partial x_{2}}, \quad \frac{\partial u_{2}}{\partial x_{1}}=-\frac{\partial u_{1}}{\partial x_{2}} .
$$

These conditions (or their extensions to the curvilinear case) can be approximated with centered differences and used to give equations for $\mathbf{u}_{-1, \mathbf{j}}$. Use of a straightforward centered approximation to these equations will, however, lead to a scheme which is not stable when the ratio $\lambda / \mu$ becomes large [24]. An approximation of the traction boundary condition that is stable for all ratios can be determined by the summation-byparts approach (see [33]) which leads to a scheme with an energy estimate. The conclusion is that the centered approximation in (17) to the mixed derivatives should be changed at the boundary $i_{1}=0$, say, by replacing the centered operator $D_{01}$ with the one-sided operator $D_{+1}$. This change, together with centered approximations to the remaining derivatives in the traction boundary condition, leads to a stable, secondorder accurate, and self-adjoint scheme. (See [30] for the full details.)

The slip-wall boundary conditions in (6) combine aspects of the displacement and traction boundary conditions. For this case, the normal component of the displacement is given on the boundary, while the $n_{d}-1$ tangential components of the displacement satisfy traction-like boundary conditions. The boundary conditions are applied in two stages. First, the displacement-like boundary condition is imposed on the normal component of the displacement, and then discrete values at ghost points are obtained using the $n_{d}-1$ Neumann conditions from the traction-like boundary conditions together with an extrapolation of the normal component of displacement. 


\section{Appendix A.2. Boundary conditions for the first-order system}

Application of the boundary conditions for the FOS scheme is somewhat more involved than that for the SOS scheme due to the increased number of dependent variables. There are three types of conditions we apply. The first type are the physical boundary conditions, either (4), (5) or (6), and boundary conditions derived from these by taking derivatives with respect to time (denoted by dot superscripts in the discussion that follows). The second type are compatibility conditions (which are numerical boundary conditions) that are derived from the governing equations and the boundary conditions. For example, the momentum equation in (1) applied on the boundary $r_{1}=0$ with the displacement boundary condition in (4) gives the compatibility condition

$$
\nabla_{h} \cdot \boldsymbol{\sigma}_{0, \mathbf{j}}^{n}=\rho \ddot{\mathbf{g}}_{d}\left(\mathbf{x}_{0, \mathbf{j}}, t_{n}\right)-\rho \mathbf{f}\left(\mathbf{x}_{0, \mathbf{j}}, t_{n}\right),
$$

where $\nabla_{h}$. denotes a second-order accurate approximation to the divergence operator, and the (known) accelerations $\ddot{\mathbf{g}}_{d}$ and $\mathbf{f}$ are evaluated at $\mathbf{x}_{0, \mathbf{j}}$ and $t=t_{n}$. The third type are extrapolation conditions, e.g. the third-order extrapolation defined previously in (A.1).

\begin{tabular}{|c|c|c|c|}
\hline stage & condition & type & assigns \\
\hline $1 \mathrm{a}$ & $\mathbf{u}_{0, \mathbf{j}}=\mathbf{g}_{d}$ & physical & $\mathbf{u}_{0, \mathbf{j}}$ \\
$1 \mathrm{~b}$ & $\mathbf{v}_{0, \mathbf{j}}=\dot{\mathrm{g}}_{d}$ & physical & $\mathbf{v}_{0, \mathbf{j}}$ \\
2 & $\mathbf{w}_{-1, \mathbf{j}}=\mathcal{E}_{+1}^{(3)} \mathbf{w}_{0, \mathbf{j}}$ & extrapolation & $\mathbf{w}_{-1, \mathbf{j}}$ \\
3 & $\nabla_{h} \cdot \boldsymbol{\sigma}_{0, \mathbf{j}}=\rho \ddot{\mathbf{g}}_{d}-\rho \mathbf{f}$ & compatibility & $\mathbf{n} \cdot \boldsymbol{\sigma}-1, \mathbf{j}$ \\
4 & $\mathbf{w}_{-2, \mathbf{j}}=\mathcal{E}_{+1}^{(3)} \mathbf{w}_{-1, \mathbf{j}}$ & extrapolation & $\mathbf{w}_{-2, \mathbf{j}}$ \\
\hline
\end{tabular}

Figure A.33: Implementation of displacement boundary conditions for the FOS scheme at the boundary face $\mathbf{r}=\left(0, r_{2}, r_{3}\right)$ with index $\mathbf{i}=(0, \mathbf{j})$, where $\mathbf{j}=\left(i_{2}, i_{3}\right)$. The values of the solution on the boundary and ghost points are assigned in the order given.

\begin{tabular}{|c|c|c|c|}
\hline stage & condition & type & assigns \\
\hline 1 & $\mathbf{n} \cdot \boldsymbol{\sigma}_{0, \mathbf{j}}=\mathbf{g}_{t}$ & physical & $\mathbf{n} \cdot \boldsymbol{\sigma}_{0, \mathbf{j}}$ \\
2 & $\mathbf{w}-1, \mathbf{j}=\mathcal{E}_{+1}^{(3)} \mathbf{w}_{0, \mathbf{j}}$ & extrapolation & $\mathbf{w}_{-1, \mathbf{j}}$ \\
$3 \mathrm{a}$ & $\mathbf{n} \cdot \mathcal{S}\left(\nabla_{h} \mathbf{u}_{0, \mathbf{j}}\right)=\mathbf{g}_{t}$ & compatibility & $\mathbf{u}-1, \mathbf{j}$ \\
$3 \mathrm{~b}$ & $\mathbf{n} \cdot \mathcal{S}\left(\nabla_{h} \mathbf{v}_{0, \mathbf{j}}\right)=\dot{\mathbf{g}}_{t}$ & compatibility & $\mathbf{v}_{-1, \mathbf{j}}$ \\
4 & $\boldsymbol{\tau}_{m} \cdot \boldsymbol{\sigma}_{0, \mathbf{j}}=\boldsymbol{\tau}_{m} \cdot \mathcal{S}\left(\nabla_{h} \mathbf{u}_{0, \mathbf{j}}\right)$ & compatibility & $\boldsymbol{\tau}_{m} \cdot \boldsymbol{\sigma}_{0, \mathbf{j}}$ \\
5 & $\boldsymbol{\sigma}_{-1, \mathbf{j}}=\mathcal{E}_{+1}^{(3)} \boldsymbol{\sigma}_{0, \mathbf{j}}$ & extrapolation & $\boldsymbol{\sigma}_{-1, \mathbf{j}}$ \\
6 & $\mathbf{w}_{-2, \mathbf{j}}=\mathcal{E}_{+1}^{(3)} \mathbf{w}_{-1, \mathbf{j}}$ & extrapolation & $\mathbf{w}_{-2, \mathbf{j}}$ \\
\hline
\end{tabular}

Figure A.34: Implementation of traction boundary conditions for the FOS scheme at the boundary face $\mathbf{r}=\left(0, r_{2}, r_{3}\right)$ with index $\mathbf{i}=(0, \mathbf{j})$, where $\mathbf{j}=\left(i_{2}, i_{3}\right)$. The values of the solution on the boundary and ghost points are assigned in the order given.

\begin{tabular}{|c|c|c|c|}
\hline stage & condition & type & assigns \\
\hline 1a & $\mathbf{n} \cdot \mathbf{u}_{0, \mathbf{j}}=g_{s}$ & physical & $\mathbf{n} \cdot \mathbf{u}_{0, \mathbf{j}}$ \\
1b & $\mathbf{n} \cdot \mathbf{v}_{0, \mathbf{j}}=\dot{g}_{s}$ & physical & $\mathbf{n} \cdot \mathbf{v}_{0, \mathbf{j}}$ \\
1c & $\mathbf{n} \cdot \boldsymbol{\sigma}_{0, \mathbf{j}} \cdot \boldsymbol{\tau}_{\alpha}=g_{s, \alpha}$ & physical & $\mathbf{n} \cdot \boldsymbol{\sigma}_{0, \mathbf{j}} \cdot \boldsymbol{\tau}_{\alpha}$ \\
2 & $\mathbf{w}-1, \mathbf{j}=\mathcal{E}_{+1}^{(3)} \mathbf{w}_{0, \mathbf{j}}$ & extrapolation & $\mathbf{w}_{-1, \mathbf{j}}$ \\
3a & $\mathbf{n} \cdot \mathcal{S}\left(\nabla_{h} \mathbf{u}_{0, \mathbf{j}}\right) \cdot \boldsymbol{\tau}_{\alpha}=g_{s, \alpha}$ & compatibility & $\boldsymbol{\tau}_{\alpha} \cdot \mathbf{u}_{-1, \mathbf{j}}$ \\
3b & $\mathbf{n} \cdot \mathcal{S}\left(\nabla_{h} \mathbf{v}_{0, \mathbf{j}}\right) \cdot \boldsymbol{\tau}_{\alpha}=\dot{g}_{s, \alpha}$ & compatibility & $\boldsymbol{\tau}_{\alpha} \cdot \mathbf{v}_{-1, \mathbf{j}}$, \\
3c & $\boldsymbol{\sigma}_{0, \mathbf{j}}=\mathcal{S}\left(\nabla_{h} \mathbf{u}_{0, \mathbf{j}}\right)$ & compatibility & $\boldsymbol{\sigma}_{0, \mathbf{j}}$ \\
4 & $\boldsymbol{\sigma}_{-1, \mathbf{j}}=\mathcal{E}_{+1}^{(3)} \boldsymbol{\sigma}_{0, \mathbf{j}}$ & extrapolation & $\boldsymbol{\sigma}_{-1, \mathbf{j}}$ \\
5 & $\mathbf{w}_{-2, \mathbf{j}}=\mathcal{E}_{+1}^{(3)} \mathbf{w}_{-1, \mathbf{j}}$ & extrapolation & $\mathbf{w}_{-2, \mathbf{j}}$ \\
\hline
\end{tabular}

Figure A.35: Implementation of slip-wall boundary conditions for the FOS scheme at the boundary face $\mathbf{r}=\left(0, r_{2}, r_{3}\right)$ with index $\mathbf{i}=(0, \mathbf{j})$, where $\mathbf{j}=\left(i_{2}, i_{3}\right)$. The values of the solution on the boundary and ghost points are assigned in the order given.

Figures A.33, A.34 and A.35 outline our implementation of the displacement, traction and slip-wall boundary conditions, respectively, for the boundary $r_{1}=0$. For each case, the boundary conditions are implemented in stages starting with the particular physical boundary conditions and then followed by compatibility and/or extrapolation conditions. In the figures, we let $\mathbf{w}=[\mathbf{u}, \mathbf{v}, \boldsymbol{\sigma}]^{T}$ now denote the vector of all dependent variables, and let

$$
\boldsymbol{\sigma}=\mathcal{S}(\nabla \mathbf{u})=\lambda \nabla \cdot \mathbf{u}+\mu\left(\nabla \mathbf{u}+\nabla \mathbf{u}^{T}\right)
$$

denote the stress-strain relation. The stages in the figures indicate the order in which the conditions are applied. For example, physical boundary conditions for the displacement are applied at stages 1a and 1b of 
Figure A.33 which assigns (overwrites) values of displacement and velocity on the boundary. Third-order extrapolation is then used to obtain values for all components of the solution in the first ghost line at $\mathbf{i}=(-1, \mathbf{j})$. The compatibility condition at stage 3 uses (A.2), which is

$$
\nabla_{h} \cdot \boldsymbol{\sigma}_{0, \mathbf{j}}^{n}=\frac{1}{J_{0, \mathbf{j}}} \sum_{\alpha=1}^{n_{d}} D_{0, \alpha}\left(J_{0, \mathbf{j}} \nabla_{\mathbf{x}} r_{\alpha} \cdot \boldsymbol{\sigma}_{0, \mathbf{j}}^{n}\right)=\rho \ddot{\mathbf{g}}_{d}\left(\mathbf{x}_{0, \mathbf{j}}, t_{n}\right)-\rho \mathbf{f}\left(\mathbf{x}_{0, \mathbf{j}}, t_{n}\right)
$$

The $n_{d}$ components of (A.4) are solved for the $n_{d}$ components of $\mathbf{n} \cdot \boldsymbol{\sigma}_{-1, \mathbf{j}}^{n}$ (noting that $\mathbf{n}=-\nabla_{\mathbf{x}} r_{1} /\left|\nabla_{\mathbf{x}} r_{1}\right|$ ), and then the values of $\boldsymbol{\sigma}_{-1, \mathbf{j}}^{n}$ are adjusted so that these conditions on the normal components are satisfied. The last stage in Figure A.33 involves a final extrapolation of all components of the solution to the second ghost line at $\mathbf{i}=(-2, \mathbf{j})$. The stages given in Figures A.34 and A.35 indicate the steps taken to implement the traction and slip-wall boundary conditions, respectively, and these are performed in a manner similar to that discussed for the displacement boundary conditions.

\section{Appendix B. Artificial dissipation for the second-order system}

In this appendix we show how the artificial dissipation term (19) acts to stabilize the SOS scheme. Note that to avoid solving an implicit system, the dissipation term in not centered in time. This leads to the question of whether this one-sided approximation has any effect on the ability of the dissipation to stabilize the scheme. We consider a periodic problem on the unit interval $[0,1]$. Let $u_{i}^{n} \approx u\left(x_{i}, t^{n}\right)$ be the discrete approximation to the solution with $x_{i}=i h, t^{n}=n \Delta t$ and $h=1 / N$. Define the difference operators in space $D_{+} u_{i}^{n}=\left(u_{i+1}^{n}-u_{i}^{n}\right) / h, D_{-} u_{i}^{n}=\left(u_{i}^{n}-u_{i-1}^{n}\right) / h$, and the difference operators in time $D_{+t} u_{i}^{n}=\left(u_{i}^{n+1}-u_{i}^{n}\right) / \Delta t$, $D_{-t} u_{i}^{n}=\left(u_{i}^{n}-u_{i}^{n-1}\right) / \Delta t$ and $D_{0 t} u_{i}^{n}=\left(u_{i}^{n+1}-u_{i}^{n-1}\right) /(2 \Delta t)$. The fully discrete scheme we consider for the second-order wave equation with dissipation of order $d=2 p$ is

$$
D_{+t} D_{-t} u_{i}^{n}=c^{2} D_{+} D_{-} u_{i}^{n}-\alpha\left(-\Delta_{+} \Delta_{-}\right)^{p} D_{-t} u_{i}^{n}, \quad i=0,1,2, \ldots, N-1,
$$

where $c$ is the wave speed and $\alpha$ is the coefficient of the artificial dissipation. The solution is assumed to be periodic in space with $u_{i+N}^{n}=u_{i}^{n}$. Define the discrete inner product and norm (for real valued grid functions) as

$$
\left(u_{i}^{n}, v_{i}^{n}\right)_{h}=\sum_{i=0}^{N-1} u_{i}^{n} v_{i}^{n} h, \quad\left\|u_{i}^{n}\right\|_{h}^{2}=\left(u_{i}^{n}, u_{i}^{n}\right)_{h}
$$

Taking the inner product of $D_{0 t} u_{i}^{n}$ with (B.1) gives

$$
\left(D_{0 t} u_{i}^{n}, D_{+t} D_{-t} u_{i}^{n}\right)_{h}=\left(D_{0 t} u_{i}^{n}, c^{2} D_{+} D_{-} u_{i}^{n}\right)_{h}-\alpha\left(D_{0 t} u_{i}^{n},\left(-\Delta_{+} \Delta_{-}\right)^{p} D_{-t} u_{i}^{n}\right)_{h}
$$

The different terms in the above expression can be manipulated making use of summation by parts identities [33],

$$
\begin{aligned}
\left(D_{0 t} u_{i}^{n}, D_{+t} D_{-t} u_{i}^{n}\right)_{h} & =\frac{1}{2 \Delta t}\left[\left\|D_{+t} u_{i}^{n}\right\|_{h}^{2}-\left\|D_{+t} u_{i}^{n-1}\right\|_{h}^{2}\right] \\
\left(D_{0 t} u_{i}^{n}, c^{2} D_{+} D_{-} u_{i}^{n}\right)_{h} & =-\frac{c^{2}}{2 \Delta t}\left(D_{+} u_{i}^{n+1}, D_{+} u_{i}^{n}\right)_{h}+\frac{c^{2}}{2 \Delta t}\left(D_{+} u_{i}^{n}, D_{+} u_{i}^{n-1}\right)_{h} \\
c^{2}\left(D_{+} u_{i}^{n+1}, D_{+} u_{i}^{n}\right)_{h} & =c^{2}\left\|D_{+} u_{i}^{n+\frac{1}{2}}\right\|_{h}^{2}-\frac{\Delta t^{2}}{4}\left(D_{+t} u_{i}^{n}, c^{2} D_{+} D_{-} D_{+t} u_{i}^{n}\right)_{h} \\
\left(D_{0 t} u_{i}^{n},\left(-\Delta_{+} \Delta_{-}\right)^{p} D_{-t} u_{i}^{n}\right)_{h} & =\left(D_{0 t} u_{i}^{n},\left(-\Delta_{+} \Delta_{-}\right)^{p} D_{0 t} u_{i}^{n}\right)_{h}-\frac{\Delta t}{2}\left(D_{0 t} u_{i}^{n},\left(-\Delta_{+} \Delta_{-}\right)^{p} D_{+t} D_{-t} u_{i}^{n}\right)_{h} \\
& =\left\|D_{0 t} \Delta_{+}^{p} u_{i}^{n}\right\|_{h}^{2}-\frac{\Delta t}{2}\left(D_{0 t} u_{i}^{n},\left(-\Delta_{+} \Delta_{-}\right)^{p} D_{+t} D_{-t} u_{i}^{n}\right)_{h}
\end{aligned}
$$

where $u_{i}^{n+\frac{1}{2}} \equiv \frac{1}{2}\left(u_{i}^{n+1}+u_{i}^{n}\right)$. This leads to the energy estimate

$$
\frac{\mathcal{E}_{h}^{n+\frac{1}{2}}-\mathcal{E}_{h}^{n-\frac{1}{2}}}{\Delta t}=-\alpha\left\|D_{0 t} \Delta_{+}^{p} u_{i}^{n}\right\|_{h}^{2}
$$


where the discrete energy $\mathcal{E}_{h}^{n+\frac{1}{2}}$ is given by

$$
\mathcal{E}_{h}^{n+\frac{1}{2}}=\frac{1}{2}\left(D_{+t} u_{i}^{n},\left\{1+\frac{c^{2} \Delta t^{2}}{4}\left(D_{+} D_{-}\right)-\frac{\alpha \Delta t}{2}\left(-\Delta_{+} \Delta_{-}\right)^{p}\right\} D_{+t} u_{i}^{n}\right)_{h}+\frac{c^{2}}{2}\left\|D_{+} u_{i}^{n+\frac{1}{2}}\right\|_{h}^{2} .
$$

This is an approximation to the continuous energy $\mathcal{E}=\frac{1}{2}\|\partial u / \partial t\|^{2}+\frac{c^{2}}{2}\|\partial u / \partial x\|^{2}$. The first term in the expression (B.5) for the discrete energy $\mathcal{E}_{h}^{n+\frac{1}{2}}$ will be non-negative provided $\Delta t$ satisfies a CFL like constraint (this can be seen by taking the discrete Fourier transform). Thus, provided $\Delta t$ satisfies this constraint, the energy estimate (B.4) shows that the scheme will be stable for $\alpha \geq 0$ and when $\alpha>0$ the energy will decay with higher wave numbers being damped more rapidly.

\section{Appendix C. The method of analytic solutions}

The method of analytic solutions is a useful technique for constructing exact solutions of initial-boundaryvalue problems for partial differential equations for the purpose of checking the behavior and accuracy of the numerical implementation of a problem. This method, also known as the method of manufactured solutions [37] or twilight-zone forcing [7], adds forcing functions to the governing equations and boundary conditions. These forcing functions are specified so that a chosen function, $\bar{u}(\mathbf{x}, t)$, becomes the exact solution of the forced equations, and thus the error in the discrete solution can be computed exactly.

As an illustration of the technique, let us consider solving an initial-boundary-value-problem (IBVP) for the equations of linear elasticity given in (1) and (2) for a chosen domain $\Omega$. The second-order form of the equations, upon elimination of the stress, is

$$
\rho \mathbf{u}_{t t}=(\lambda+\mu) \nabla(\nabla \cdot \mathbf{u})+\mu \nabla^{2} \mathbf{u}+\rho \mathbf{f}, \quad \text { for } \mathbf{x} \in \Omega, t>0,
$$

with initial conditions and boundary conditions taken to be

$$
\begin{aligned}
& \mathbf{u}(\mathbf{x}, 0)=\mathbf{u}_{0}(\mathbf{x}), \quad \mathbf{u}_{t}(x, 0)=\mathbf{v}_{0}(\mathbf{x}), \quad \text { for } \mathbf{x} \in \Omega, \\
& \mathbf{u}(\mathbf{x}, t)=\mathbf{g}_{d}(\mathbf{x}, t), \quad \text { for } \mathbf{x} \in \partial \Omega, t>0 \text {. }
\end{aligned}
$$

Any given smooth function, $\overline{\mathbf{u}}(\mathbf{x}, t)$, will be an exact solution of the IBVP if we set the forcing function, initial displacement and velocity, and the displacement on the boundary to be

$$
\begin{aligned}
\mathbf{f}(\mathbf{x}, t) & =\overline{\mathbf{u}}_{t t}-\left[(\lambda+\mu) \nabla(\nabla \cdot \overline{\mathbf{u}})+\mu \nabla^{2} \overline{\mathbf{u}}\right] / \rho, & & \text { for } \mathbf{x} \in \Omega, t>0, \\
\mathbf{u}_{0}(\mathbf{x}) & =\overline{\mathbf{u}}(\mathbf{x}, 0), \quad \mathbf{v}_{0}(\mathbf{x})=\overline{\mathbf{u}}_{t}(\mathbf{x}, 0), & & \text { for } \mathbf{x} \in \Omega, \\
\mathbf{g}_{d}(\mathbf{x}, t) & =\overline{\mathbf{u}}(\mathbf{x}, t), & & \text { for } \mathbf{x} \in \partial \Omega, t>0 .
\end{aligned}
$$

The illustration here considers an IBVP for the second-order formulation of the equations with displacement boundary conditions, but a similar approach can be used for the first-order formulation and for other types of boundary conditions.

In our numerical implementation, we have a number of choices available for $\overline{\mathbf{u}}(\mathbf{x}, t)$ including polynomials, trigonometric and exponential functions, among others. For example, trigonometric functions are used for the results in Sections 6.1 and 6.2, while exponential functions are used for the results in Section 6.3. For the case of the second-order form of the governing equations, we only require a chosen function for displacement (as in IBVP above). For the case of the first-order equations in (7), we also require functions for velocity and stress. These functions could be derived from the one for displacement, but we choose instead to make independent choices and include forcing functions in the rate equations for displacement and stress so that the chosen functions for displacement, velocity and stress are exact solutions of the first-order form of the governing equations.

\section{Appendix D. Exact solutions to the elastic wave equation for an annulus and a sphere.}

In this appendix we define the exact solutions that are used in Section 6.4 for the solid annulus and in Section 6.5 for the solid sphere. 
Appendix D.1. Vibrational modes of an elastic annulus - exact solutions

We consider a two-dimensional annular domain $\Omega_{A}$ with inner radius $R_{a}$ and outer radius $R_{b}$. Solutions of the governing equations that depend only on the radius $r$ and time $t$ satisfy

$$
\rho \frac{\partial^{2}}{\partial t^{2}} u_{r}=(\lambda+2 \mu) \frac{\partial}{\partial r}\left(\frac{1}{r} \frac{\partial}{\partial r}\left(r u_{r}\right)\right), \quad \text { for } R_{a}<r<R_{b}, t>0,
$$

where $u_{r}(r, t)$ denotes the radial component of displacement. We consider solutions satisfying the displacement boundary conditions

$$
u_{r}(r, t)=0, \quad \text { at } r=R_{a} \text { and } R_{b},
$$

or the traction boundary conditions

$$
\frac{\lambda}{r} \frac{\partial}{\partial r}\left(r u_{r}\right)+2 \mu \frac{\partial}{\partial r} u_{r}=-p(r, t), \quad \text { at } r=R_{a} \text { and } R_{b},
$$

where $p$ is a given pressure on the boundary. Time-independent solutions to (D.1) have the form

$$
u_{r}^{(0)}(r)=A_{0} r+\frac{B_{0}}{r},
$$

where $A_{0}$ and $B_{0}$ are constants of integration. For the case of the homogeneous boundary conditions in (D.2) only the trivial solution with $A_{0}=B_{0}=0$ exists. For the traction boundary conditions in (D.3) with a constant pressures $P_{a}$ and $P_{b}$ on the inner and outer boundaries, respectively, a nontrivial solution exists with integration constants

$$
A_{0}=\frac{P_{b} R_{b}^{2}-P_{a} R_{a}^{2}}{2(\lambda+\mu)\left(R_{a}^{2}-R_{b}^{2}\right)}, \quad B_{0}=\frac{\left(P_{b}-P_{a}\right) R_{a}^{2} R_{b}^{2}}{2 \mu\left(R_{a}^{2}-R_{b}^{2}\right)} .
$$

(see Love [38], page 144). Time-harmonic solutions to (D.1) have the form

$$
u_{r}^{(n)}(r, t)=\left[A_{n} J_{1}\left(\alpha_{n} r\right)+B_{n} Y_{1}\left(\alpha_{n} r\right)\right] \cos \left(\omega_{n} t\right), \quad n=1,2, \ldots,
$$

where $n$ denotes a vibrational mode, $J_{1}$ and $Y_{1}$ are Bessel functions of the first and second kind, respectively, of order one and $\alpha_{n}$ is related to the frequency $\omega_{n}$ by $\alpha_{n}^{2}=\rho \omega_{n}^{2} /(\lambda+2 \mu)$. The eigenvalues, $\alpha_{n}, n=1,2, \ldots$, are the roots of the equation

$$
G_{1}\left(\alpha R_{a}\right) H_{1}\left(\alpha R_{b}\right)-G_{1}\left(\alpha R_{b}\right) H_{1}\left(\alpha R_{a}\right)=0,
$$

where $G_{1}(r)=J_{1}(r)$ and $H_{1}(r)=Y_{1}(r)$, for the case of displacement boundary conditions, and $G_{1}(r)=$ $(\lambda+2 \mu) r J_{1}^{\prime}(r)+\lambda J_{1}(r), H_{1}(r)=(\lambda+2 \mu) r Y_{1}^{\prime}(r)+\lambda Y_{1}(r)$, for the case of (homogeneous) traction boundary conditions. The ratio of the constants $A_{n}$ and $B_{n}$ is given by $B_{n} / A_{n}=-G_{1}\left(\alpha_{n} R_{a}\right) / H_{1}\left(\alpha_{n} R_{a}\right)$ for both cases.

\section{Appendix D.2. Vibrational modes of an elastic sphere - exact solutions}

We describe some exact solutions for small amplitude vibrations of a solid elastic sphere. This is a classical problem in linear elasticity, and exact solutions are discussed in Lamb [39] and Love [38], for example. Solutions of the linear problem are expressed as a sum of vibrational modes, and we consider one such mode (a so-called solution of the second class) for the purpose of verification of our numerical methods.

For a sphere of radius $R$, the $j^{\text {th }}$ component of displacement of the $n^{\text {th }}$ mode in Cartesian coordinates $\left(x_{1}, x_{2}, x_{3}\right)$ can be written in the form

$$
\begin{aligned}
u_{j}^{(n)}=A_{n} & \cos \left(\omega_{n} t\right)\left\{-\frac{1}{\alpha_{n}^{2}}\left(\frac{\partial \zeta_{n}}{\partial x_{j}}\right) \psi_{n}\left(\alpha_{n} \varrho\right)-\left(x_{j} \zeta_{n}\right) \psi_{n+1}\left(\alpha_{n} \varrho\right)\right. \\
& \left.+C_{n}\left[\frac{1}{\kappa_{n}^{2}}\left(\frac{\partial \zeta_{n}}{\partial x_{j}}\right) \psi_{n-1}\left(\kappa_{n} \varrho\right)-\frac{n}{n+1}\left(r^{2} \frac{\partial \zeta_{n}}{\partial x_{j}}-(2 n+1) x_{j} \zeta_{n}\right) \psi_{n+1}\left(\kappa_{n} \varrho\right)\right]\right\},
\end{aligned}
$$

where $A_{n}$ is the amplitude of the mode, $\omega_{n}$ is its frequency, $\alpha_{n}$ and $\kappa_{n}$ are constants related to the frequency by

$$
\alpha_{n}^{2}=\frac{\rho \omega_{n}^{2}}{\lambda+2 \mu}, \quad \kappa_{n}^{2}=\frac{\rho \omega_{n}^{2}}{\mu},
$$


$C_{n}$ is a constant, and $\psi_{n}$ and $\zeta_{n}$ are functions given by

$$
\psi_{n}(\varrho)=\left(\frac{1}{\varrho} \frac{d}{d \varrho}\right)^{n}\left(\frac{\sin \varrho}{\varrho}\right), \quad \zeta_{n}(\varrho, \theta, \phi)=\varrho^{n} e^{i m \theta} P_{n}^{m}(\cos \phi) .
$$

Here, $\psi_{n}$ is related to the spherical Bessel function (of the first kind) of order $n, \zeta_{n}$ is the solid spherical harmonic of order $n$, and $P_{n}^{m}$ is the associated Legendre function of order $n$ and degree $m$. These functions are written in terms of the usual spherical polar coordinates $(\varrho, \theta, \phi)$, where $0 \leq \varrho \leq R, 0 \leq \theta \leq 2 \pi$, and $0 \leq \phi \leq \pi$ for the solid sphere. Application of a stress-free boundary condition at $r=R$ provides constraints that determine an infinite number of $\left(\kappa_{n}, C_{n}\right)$ pairs for each $n$ (independent of $m$ ), see [38]. Corresponding values for $\alpha_{n}$ and $\omega_{n}$ may then be found using (D.8).

Within the class of solutions given in (D.7), we consider the mode $n=2$ with $m=0$, i.e. spheroidal vibrations. For this case, the solid spherical harmonic function in (D.9) becomes independent of $\theta$, and it takes the simple form

$$
\zeta_{2}=\frac{\varrho^{2}}{2}\left(3 \cos ^{2} \phi-1\right)=x_{3}^{2}-\frac{1}{2}\left(x_{1}^{2}+x_{2}^{2}\right),
$$

using $\varrho^{2}=x_{1}^{2}+x_{2}^{2}+x_{3}^{2}$ and $\cos \phi=x_{3} / \varrho$. The formula for the solid spherical harmonic in (D.10) may now be used in (D.7) to give the solution mode

$$
u_{j}^{(2)}=A_{2} \cos \left(\omega_{2} t\right) \hat{u}_{j}^{(2)}, \quad j=1,2,3,
$$

where

$$
\hat{u}_{j}^{(2)}=x_{j}\left\{\frac{1}{\alpha_{2}^{2}} \psi_{2}\left(\alpha_{2} \varrho\right)-\frac{1}{2}\left(2 x_{3}^{2}-r^{2}\right) \psi_{3}\left(\alpha_{2} \varrho\right)-C_{2}\left[\frac{1}{\kappa_{2}^{2}} \psi_{1}\left(\kappa_{2} \varrho\right)+\frac{1}{3}\left(8 x_{3}^{2}-7 r^{2}\right) \psi_{3}\left(\kappa_{2} \varrho\right)\right]\right\},
$$

for $j=1$ and 2 , and

$$
\hat{u}_{3}^{(2)}=-x_{3}\left\{\frac{2}{\alpha_{2}^{2}} \psi_{2}\left(\alpha_{2} \varrho\right)+\frac{1}{2}\left(2 x_{3}^{2}-r^{2}\right) \psi_{3}\left(\alpha_{2} \varrho\right)-C_{2}\left[\frac{2}{\kappa_{2}^{2}} \psi_{1}\left(\kappa_{2} \varrho\right)+\frac{1}{3}\left(6 x_{3}^{2}-7 r^{2}\right) \psi_{3}\left(\kappa_{2} \varrho\right)\right]\right\} .
$$

Here, $\psi_{1}, \psi_{2}$ and $\psi_{3}$ are given by the formula in (D.9) and $r^{2}=x_{1}^{2}+x_{2}^{2}$. Three values for $\left(\kappa_{2}, C_{2}\right)$, corresponding to the lowest three frequencies of vibration for this mode, are listed in Figure D.36 for the case $\lambda=\mu$. The solution mode given by (D.11) is axisymmetric, and corresponds to a sphere which elongates and compresses periodically along the $x_{3}$-axis.

\begin{tabular}{|c|c|c|}
\hline & $\kappa_{2} R$ & $C_{2}$ \\
\hline 1 & 2.63986927790186 & -2.61595562778538 \\
2 & 4.86527284993742 & -1.89108063594100 \\
3 & 8.32919545905501 & 3.21915564815474 \\
\hline
\end{tabular}

Figure D.36: Leading three values for $\left(\kappa_{2}, C_{2}\right)$ for the vibration mode with $n=2$ given in (D.11) for a sphere of radius $R$.

\section{References}

[1] G. C. Cohen, Higher-Order Numerical Methods for Transient Wave Equations, Springer, New York, 2002.

[2] M. Dumbser, M. Käser, E. F. Toro, An arbitrary high-order discontinuous Galerkin method for elastic waves on unstructured meshes V. Local time stepping and p-adaptivity, Geophysical Journal International 171 (2007) 695-717.

[3] V. Etienne, E. Chaljub, J. Virieux, N. Glinsky, An hp-adaptive discontinuous Galerkin finite-element method for 3-d elastic wave modelling, Geophysical Journal International 183 (2010) 941-962.

[4] L. C. Wilcox, G. Stadler, C. Burstedde, O. Ghattas, A high-order discontinuous Galerkin method for wave propagation through coupled elastic-acoustic media, J. Comput. Phys. 229 (2010) 9373 - 9396.

[5] W. D. Henshaw, A high-order accurate parallel solver for Maxwell's equations on overlapping grids, SIAM J. Sci. Comput. 28 (2006) 1730-1765. 
[6] J. W. Banks, W. D. Henshaw, D. W. Schwendeman, Deforming composite grids for solving fluid structure problems, J. Comput. Phys. 231 (2012) 3518 - 3547.

[7] G. S. Chesshire, W. D. Henshaw, Composite overlapping meshes for the solution of partial differential equations, J. Comput. Phys. 90 (1990) 1-64.

[8] W. D. Henshaw, D. W. Schwendeman, An adaptive numerical scheme for high-speed reactive flow on overlapping grids, J. Comput. Phys. 191 (2003) 420-447.

[9] J. W. Banks, D. W. Schwendeman, A. K. Kapila, W. D. Henshaw, A high-resolution Godunov method for compressible multi-material flow on overlapping grids, J. Comput. Phys. 223 (2007) 262-297.

[10] W. D. Henshaw, D. W. Schwendeman, Parallel computation of three-dimensional flows using overlapping grids with adaptive mesh refinement, J. Comput. Phys. 227 (2008) 7469-7502.

[11] W. M. Chan, Overset grid technology development at NASA Ames Research Center, Comput. Fl. 38 (2009) 496-503.

[12] D. W. Schwendeman, A. K. Kapila, W. D. Henshaw, A study of detonation diffraction and failure for a model of compressible two-phase reactive flow, Combust. Theory and Modeling 14 (2010) 331-366.

[13] W. D. Henshaw, K. K. Chand, A composite grid solver for conjugate heat transfer in fluid-structure systems, J. Comput. Phys. 228 (2009) 3708-3741.

[14] X. Lin, J. Ballmann, A numerical scheme for axisymmetric elastic waves in solids, Wave Motion 21 (1995) 115-126.

[15] R. J. Niethammer, K. S. Kim, J. Ballmann, Numerical simulation of shock waves in linear-elastic plates with curvilinear boundaries and material interfaces, International Journal of Impact Engineering 16 (1995) 1711-725.

[16] D. Appelö, S. Nilsson, N. A. Petersson, B. Sjögreen, A stable finite difference method for the elastic wave equation on complex domains with free surface boundary conditions, in: Proceedings of Waves 2007, Reading, United Kingdom.

[17] G. Browning, H.-O. Kreiss, J. Oliger, Mesh refinement, Mathematics of Computation 27 (1973) 29-39.

[18] M. Ciment, Stable difference schemes with uneven mesh spacings, Math. Comp. 25 (1971) 219-226.

[19] M. J. Berger, Stability of interfaces with mesh refinement, Math. Comp. 45 (1985) 301-318.

[20] G. Starius, On composite mesh difference methods for hyperbolic differential equations, Numer. Math. 35 (1980) 241-255.

[21] L. G. M. Reyna, Part III. On Composite Meshes, Ph.D. thesis, Dept. of Applied Mathematics, California Institute of Technology, 1982.

[22] E. Pärt-Enander, B. Sjögreen, Conservative and non-conservative interpolation between overlapping grids for finite volume solutions of hyperbolic problems, Comput. Fluids 23 (1994) 551-574.

[23] F. Olsson, N. A. Petersson, Stability of interpolation on overlapping grids, Comput. Fl. 25 (1996) 583-605.

[24] A. Ilan, D. Loewenthal, Instability of finite difference schemes due to boundary conditions in elastic media, Geophysical Prospecting 24 (1976) 431-453.

[25] M. J. Berger, J. Oliger, Adaptive mesh refinement for hyperbolic partial differential equations, J. Comput. Phys. 53 (1984) 484-512.

[26] K. F. Graff, Wave Motion in Elastic Solids, Dover Publications, 1991.

[27] W. D. Henshaw, Ogen: An Overlapping Grid Generator for Overture, Research Report UCRL-MA132237, Lawrence Livermore National Laboratory, 1998. 
[28] A. Sussman, G. Agrawal, J. Saltz, A Manual for the Multiblock PARTI Runtime Primitives, Revision 4.1, Technical Report CS-TR-3070.1, University of Maryland, Department of Computer Science, 1993.

[29] W. Gropp, E. Lusk, R. Thakur, Using MPI-2: Advanced Features of the Message-Passing Interface, The MIT Press, Cambridge, MA, 1999.

[30] D. Appelö, N. A. Petersson, A stable finite difference method for the elastic wave equation on complex geometries with free surfaces, Communications in Computational Physics 5 (2009) 84-107.

[31] C. Bogey, C. Bailly, A family of low dispersive and low dissipative explicit schemes for flow and noise computations, J. Comput. Phys. 194 (2004) 194-214.

[32] S. K. Lele, Compact finite difference schemes with spectral-like resolution, J. Comput. Phys. 103 (1992) $16-42$.

[33] B. Gustafsson, H.-O. Kreiss, J. Oliger, Time Dependent Problems and Difference Methods, John Wiley and Sons Inc., 1995.

[34] J. W. Banks, T. Aslam, W. J. Rider, On sub-linear convergence for linearly degenerate waves in capturing schemes, J. Comput. Phys. 227 (2008) 6985-7002.

[35] H.-O. Kreiss, J. Oliger, Comparison of accurate methods for the integration of hyperbolic equations, Tellus 24 (1972) 199-215.

[36] A. Bayliss, K. E. Jordan, B. J. LeMesurier, E. Turkel, A fourth-order accurate finite-difference scheme for the computation of elastic waves, Bulletin of the Seismological Society of America 76 (1986) 11151132.

[37] P. J. Roache, Code verification by the method of manufactured solutions, ASME J. of Fluids Engineering $124(2002) 4-10$.

[38] A. E. H. Love, A Treatise on the Mathematical Theory of Elasticity, Dover Publications, New York, 1944.

[39] H. Lamb, On the vibrations of an elastic sphere, Proceedings of the London Mathematical Society 13 (1882) 189-212. 